Ordens parciais e aplicações

Dione Andrade Lara 
SERVIÇO DE PÓS-GRADUAÇÃO DO ICMC-USP

Data de Depósito:

Assinatura:

\title{
Ordens parciais e aplicações
}

\author{
Dione Andrade Lara
}

Orientador: Prof. Dr. Leandro Fiorini Aurichi

Dissertação apresentada ao Instituto de Ciências Matemáticas e de Computação - ICMC-USP, como parte dos requisitos para obtenção do título de Mestre em Ciências - Matemática . VERSÃO REVISADA 
Ficha catalográfica elaborada pela Biblioteca Prof. Achille Bassi e Seção Técnica de Informática, ICMC/USP, com os dados fornecidos pelo(a) autor(a)

Andrade Lara, Dione
Ordens Parciais e Aplicações / Dione Andrade Lara;
orientador Leandro Fiorini Aurichi. -- São Carlos,
2012. $133 \mathrm{p.}$
Dissertação (Mestrado - Programa de Pós-Graduação en
Matemática)-- Instituto de Ciências Matemáticas e
de Computação, Universidade de São Paulo, 2012.
1. Axioma de Martin. 2. Blumberg. 3. Hipótese de
Suslin. Jogos Topológicos. 5. Princípio Diamante.
I. Fiorini Aurichi, Leandro, orient. II. Título.


"Sonho que se sonha só. É só um sonho que se sonha só. Mas sonho que se sonha junto é realidade." Raul Seixas 



\section{Agradecimentos}

Gostaria de agradecer ao programa de pós graduação do USP-ICMC (Instituto de Matemática Aplicada e Computação). Aos professores e funcionários que contribuiram para a confecção dessa dissertação.

Ao meu orientador, Leandro Fiorini Aurichi, que acompanhou o desenvolvimento desse trabalho. Com muita paciência e dedicação tornou possível o meu aprendizado desde o básico a assuntos mais sofisticados. Incentivou as apresentações que realizei no instituto periodicamente e, além disso, tornou possível a participação na Segunda Semana de Topologia Geral e Teoria de Conjuntos (2nd Set Theory and General Topology Week) em Salvador BA. Sem falar, pela amizade e confiança em acreditar nesse aluno.

Agradeço a professora Márcia Federson que intercedeu por mim inúmeras vezes.

Aos meus amigos, Fabiana e Steve, que começaram a jornada pela pós graduação junto comigo na UFSCar. Agradeço também aos amigos Daniel (Folgado) e Flora em que tive o prazer de compartilhar os melhores anos de minha gradução e apesar da distância que nos separam hoje, mantemos os mesmos laços de antes.

Aos professores da UFSCar, Adriana Ramos, Daniel Vendrúscolo, Ivo Machado, Marcos Vinícius e Pedro Malagutti. Tive a honra de absorver uma pequena parte de seus conhecimentos, contribuindo para chegar academicamente onde cheguei. Mais que isso, foram amigos, além do conhecimento técnico, aprendi muito com as conversas fora de sala de aula.

Aos novos amigos feitos no ICMC, onde passamos dias exaustivos de estudo, dificuldade de acompanhar as disciplinas, às vezes indo mal em algumas provas, porém, nos 
encontrando em churrascos e rindo disso tudo, além das piadas sem graça que eu contava.

Aos companheiros do Bloco 36 da moradia da UFSCar, que sempre me apoiaram e dividiram alguns fardos que sozinho com certeza não iria aguentar. À Alexandre (Jesus), Carlos (Herbie), Carlos Eduardo (Carlão), Fabiano (Bino), Paulo (Sexto), Rodolfo (Xico), Rogério (Corvo), Tiago (Tiii), Victor (RIP) e Welton (Goiano) que fizeram muita diferença no período em que estive como morador ou agregado.

Aos meus amigos da minha cidade Pratápolis MG, Bruno, Danilo (Brou), Diego, Eduardo (Dudu), Rafaela, Sandro e Tharles que sempre me recebem de braços abertos quando volto para a boa e velha "Pratinha".

Às pessoas que estão bem próximas a mim, Alessandra (Flexinha), Alomir, Camilo, Lais e Rodrigo. Especialmente à dona Mailce, que além de ter muita paciência para escutar as minhas lamentações, ainda me ajudou a tomar algumas decisões nos momentos difíceis.

À minha família, que ajudou mais do que eu merecia. Sem o apoio deles não seria possível nem ir morar em Franca para fazer cursinho e poder realizar aquele sonho de passar no vestibular.

À todos os citados e as pessoas que não mencionei, mas que fizeram parte da minha trajetória, fica registrado o meu muito obrigado. Graças a vocês foi possível a confecção desse trabalho!

À CAPES pelo apoio financeiro. 


\section{Resumo}

Este trabalho é dividido em duas partes:

Na primeira, apresentaremos três axiomas extras a $Z F C$ referentes a ordens parciais, sendo eles: o Axioma de Martin $(M A)$, o princípio diamante $(\diamond)$ e a hipótese de Suslin $(S H)$.

Na segunda parte daremos algumas aplicações desses axiomas em teoria de conjuntos e em topologia geral. Começaremos falando sobre famílias dominantes e ilimitadas e observando que certas relações entre tais conceitos se diferem ao assumirmos apenas $Z F C$ ou $M A$.

Provaremos a independência da hipótese de Suslin, usando a consistência de $M A$ e de $\diamond$. Apresentaremos três jogos topológicos: sendo eles o jogo de Choquet, o jogo de Rothberger e o jogo de Menger. O ganho na linguagem de jogos é deixar algo "complicado" com uma formulação mais simples.

Analisaremos o produto de espaços que satisfazer a c.c.c. (countable chain condition) novamente sob a luz de $Z F C$ ou $M A$. Construiremos um espaço compacto e Hausdorff onde o conjunto dos naturais é denso e além disso, para toda função contínua com domínio $\mathbb{N}$ à valores num compacto, tal função admite uma extensão contínua para esse espaço. Finalmente, veremos quais condições um espaço precisa satisfazer para ser de Blumberg e uma tentativa de caracterizar tais espaços via jogos topológicos. 
Palavras-chave: Axioma de Martin; Blumberg; Hipótese de Suslin; Jogos Topológicos; Princípio Diamante. 


\section{Abstract}

This work is divided in two parts:

At first, we introduce three extra axioms to $Z F C$ related to partial orders, namely: the Martin Axiom $(M A)$, the Diamond Principle $(\diamond)$ and the Suslin hypothesis $(S H)$.

In the second part we give some applications of these axioms in set theory and general topology. We start talking about dominant and unbounded families and that certain relations between these concepts differ if we assume only $Z F C$ or $M A$.

We prove the independence of Suslin hypothesis using the consistency of $M A$ and $\diamond$. We present three topological games, the Choquet game, the Rothberger game and the Menger game. The gain in the games language is to say something "difficult" with a simpler formulation.

We analyze the product of spaces c.c.c. (countable chain condition) under $Z F C$ or $M A$. We construct a compact Hausdorff space where the natural numbers are dense and, moreover, for any continuous function with domain $\mathbb{N}$ to a compact space, such a function admits a continuous extension. Finally, we will see what conditions a space has to satisfy to be Blumberg and, an attempt to characterize spaces via topological games.

Key words: Blumberg; Diamond Principle; Martin Axiom; Suslin Hypothesis; Topological Games. 



\section{Sumário}

I Conceitos Básicos

1 Teoria dos Conjuntos e Ordens Parciais $\quad 7$

1.1 Ordens Parciais . . . . . . . . . . . . . . . . . 7

1.2 Ordinais e Cardinais . . . . . . . . . . . . . . . . . 10

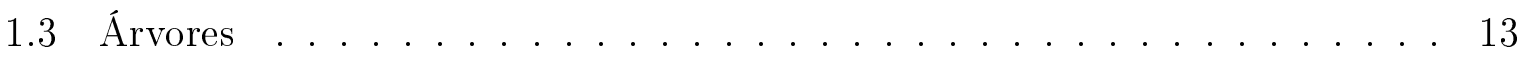

1.4 Álgebra de Boole . . . . . . . . . . . . . . . . . . . . 21

1.5 Filtros e Ultrafiltros . . . . . . . . . . . . . . . . . 23

2 Noções Básicas de Topologia $\quad 27$

3 Dois Axiomas Extras à ZFC

3.1 O Axioma de Martin . . . . . . . . . . . . . . . . 35

3.2 Clubs e Conjuntos Estacionários . . . . . . . . . . . . . 38

$3.3 \quad$ o Princípio $\diamond \ldots \ldots \ldots \ldots \ldots \ldots \ldots \ldots$

II Aplicações $\quad 47$

4 Famílias Dominantes e Famílias Ilimitadas 49

4.1 Sob $Z F C \ldots \ldots \ldots \ldots \ldots \ldots \ldots \ldots$ 
4.2 Sob o Axioma de Martin . . . . . . . . . . . . . . . . . . 52

5 A Hipótese de Suslin $\quad 55$

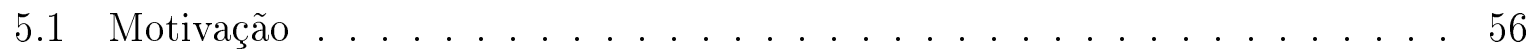

$5.2 M A$ implica $S H \ldots \ldots \ldots \ldots \ldots \ldots$

5.3 Princípio $\diamond$ implica $\neg S H \ldots \ldots \ldots \ldots \ldots$

$\begin{array}{llr}6 & \text { Jogos Topológicos } & 77\end{array}$

6.1 Jogo de Choquet (Banach Mazur) . . . . . . . . . . . 77

6.2 Jogo de Rothberger . . . . . . . . . . . . . . . . 82

6.3 Jogo de Menger . . . . . . . . . . . . . . 86

$\begin{array}{lll}7 & \text { Produto de espaços c.c.c. } & 91\end{array}$

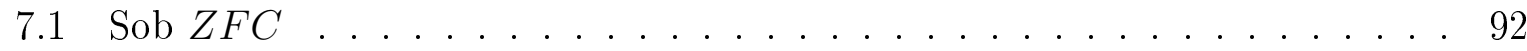

7.2 Sob o Axioma de Martin . . . . . . . . . . . . . . . . 95

7.3 Sob a negação da Hipótese de Suslin . . . . . . . . . . . . . . . . . 97

8 O espaço de Stone de uma álgebra de Boole $\quad 99$

8.1 Espaço de Stone . . . . . . . . . . . . . . . . . . . . . 99

8.2 Convergência de ultrafiltros . . . . . . . . . . . . . . 102

8.3 Compactificação de Stone-Cech dos Naturais via espaços de Stone . . . . . 103

9 Espaços de Blumberg $\quad 109$

9.1 Espaços de Blumberg . . . . . . . . . . . . . . . . 109

9.2 Um Exemplo de um Espaço Compacto Hausdorff e não Blumberg . . . . . 117

9.3 Caracterização por Jogos . . . . . . . . . . . . . . . . . . . 124 


\section{Introdução}

O conceito de conjuntos com as operações de união e intersecção é tão intuitivo como o próprio conceito de número. Durante muito tempo os matemáticos usaram essa noção intuitiva de conjuntos até que, no século XX, com o avanço das teorias abstratas começaram a aparecer diversos paradoxos que estremeceram as bases da imensa árvore matemática. Tais "anomalias" serviram para que os matemáticos abandonassem a abordagem intuitiva e "ingênua" e assumirem o rigor e a lógica formal como base para a reconstrução da matemática. A primeira "construção" da teoria de conjuntos formal, ou seja, um sistema axiomático para a matemática, foi concebida por Ernst Zermelo, Abraham Fraenkel e Thoralf Skolem no início do século XX.

Um tempo antes dessa formalização o matemático alemão Georg Cantor "arranhava" as paredes dessas limitações. Em 1878 enunciou a Hipótese do Contínuo $(\mathrm{CH})$. Cantor acreditava que $C H$ fosse verdade e tentou por muitos anos prová-la, em vão. A hipótese do contínuo tornou-se o primeiro problema na famosa lista de David Hilbert, que foi apresentado no Congresso Internacional de Matemática, no ano de 1900 em Paris. Kurt Gödel mostrou em 1940 que $C H$ não pode ser refutada sob os axiomas de ZermeloFraenkel $(Z F)$, nem mesmo adotando o axioma da escolha $(Z F C)$. Finalmente, Paul Cohen mostrou em 1963 que $C H$ não pode ser provado sob os axiomas de $Z F C$. Portanto, dizemos que $C H$ é independente de $Z F C$. Assumindo tanto $C H$ quanto a sua negação $\neg C H$ não geram contradições com $Z F C$.

Esta monografia tem por objetivo apresentar três novos axiomas referentes a ordens parciais (i.e. afirmações independentes à $Z F C$ ) que são: o Axioma de Martin, o princípio 
diamante $(\diamond)$ e a hipótese de Suslin. Além disso, exibiremos algumas aplicações desses axiomas extras à teoria de conjuntos e em topologia geral.

Os capítulos 1 e 2 consistem de pré-requisitos básicos para a compreensão dos demais capítulos. No primeiro, estabelecemos as definições e resultados básicos de teoria de conjuntos que serão usados no corpo da dissertação. Assumiremos que o leitor tenha uma certa familiaridade com a teoria de conjuntos e por isso alguns resultados básicos serão omitidos. O segundo capítulo tem como função introduzir conceitos básicos de topologia geral.

No Capítulo 3, introduziremos o Axioma de Martin $(M A)$ e faremos uma generalização do Teorema de Baire para espaços compactos Hausdorff que satisfazem c.c.c.. Posteriormente, veremos os pré-requisitos necessários para se entender o princípio diamante. Dentre eles, falaremos de clubs e de conjuntos estacionários. Como aplicação desses conceitos veremos o Lema do "Pressing Down".

No Capítulo 4, veremos o que são famílias dominantes e famílias ilimitadas. Analisaremos os menores tamanhos possíveis para tais famílias. Nas seções seguintes serão traçadas as relações de tais cardinais sob a luz de $Z F C$ ou sob $M A$.

No Capítulo 5, essencialmente definiremos a Hipótese de Suslin $(S H)$ e mostraremos que a mesma é idependente. Mostraremos que $M A$ implica $S H$ e que $\diamond$ implica em $\neg S H$.

No Capítulo 6, falaremos sobre Jogos Topológicos. Destacaremos três jogos, sendo eles o jogo de Choquet, o jogo de Rothberger e o jogo de Menger e diremos o que é um espaço ser de Choquet, ou ser de Rothberger ou de Menger. Veremos algumas aplicações interessantes, tais como Choquet implica Baire, Compacto Hausdorff implica Choquet, todo espaço de Rothberger é de Lindelöf (também Menger é de Lindelöf). Além disso, exibiremos um espaço compacto que não é de Rothberger e um espaço de Lindelöf que não é de Menger.

No Capítulo 7, veremos algumas propriedades referentes ao produto de espaços que satisfazem a c.c.c. (countable chain condition). Em $Z F C$ temos um resultado curioso: Dado o produto infinito de espaços c.c.c. basta que o produto de qualquer parte finita seja c.c.c. para que o produto todo satisfaça essa propriedade. Enquanto que, se assumirmos $M A$ o produto de espaços c.c.c sempre é c.c.c.. Tal resultado não pode ser mostrado em 
$Z F C$ apenas, pois se assumirmos a negação de $S H$ temos que existe a reta de Suslin, que é c.c.c., mas o quadrado dessa reta não o é.

O Capítulo 8, tratará da construção de um espaço compacto e Hausdorff onde o conjunto dos naturais é denso. Além disso, para toda função contínua com domínio $\mathbb{N}$ (também chamaremos de $\omega$ ) à valores num compacto, tal função admite uma extensão contínua para esse espaço. Esse espaço é conhecido como compactificado de Stone-Čech dos naturais. Na última seção veremos uma aplicação desse espaço à análise funcional.

Finalmente, no capítulo 9, veremos quais condições um espaço precisa satisfazer para ser de Blumberg. Nas duas seções seguintes tentaremos caracterizar tais espaços via o jogo de Choquet. Infelizmente Choquet não implica em Blumberg e nem vale a implicação contrária!

Para ajudar na compreensão do conteúdo descrito nesse trabalho temos o seguinte diagrama:

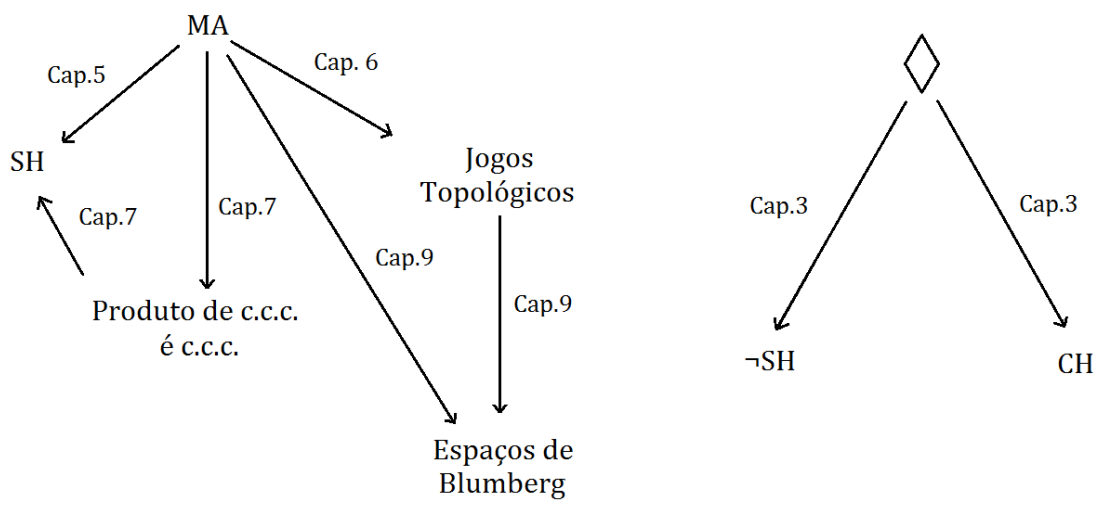

Figura 1: Diagrama das implicações dos axiomas extras 


\section{Parte I}

\section{Conceitos Básicos}





\section{Capítulo}

\section{Teoria dos Conjuntos e Ordens Parciais}

O intuito desse capítulo é estabelecer as definições e resultados básicos que serão usados no corpo da dissertação. Assumiremos que o leitor tenha uma certa familiaridade com a teoria de conjuntos e por isso alguns resultados básicos serão omitidos.

\subsection{Ordens Parciais}

Definição 1.1. Dizemos que uma relação $\leq e ́$ uma relação de pré-ordem sobre um conjunto P se são satisfeitas as seguintes condições:

(1) para todo $a \in P$ temos que $a \leq a$ (reflexiva);

(2) para todo $a \in P$, para todo $b \in P$ e para todo $c \in P$, se $a \leq b$ e $b \leq c$, então $a \leq c$ (transitiva).

$O$ conjunto $(P, \leq)$ é chamado de conjunto pré-ordenado.

(3) Se, além disso, valer:

para todo $a \in P$, para todo $b \in P$ se $a \leq b$ e $b \leq a$, então $a=b$ (antissimétrica).

então dizemos que $\leq$ é uma ordem. 
O conjunto $(P, \leq)$ é chamado de conjunto ordenado (ou parcialmente ordenado). Um conjunto $(P, \leq)$ é totalmente ordenado se para todo $a, b \in P a \leq b$ ou $b \leq a$, ou seja, todos os elementos são comparáveis.

Definição 1.2. Dizemos que uma relação < é uma relação de ordem estrita sobre $P$ se são satisfeitas as seguintes condições:

(1) para todo $a \in P$ não ocorra $a<a$ (irreflexiva);

(2) para todo $a \in P$, para todo $b \in P$ e para todo $c \in P$ se $a<b$ e $b<c$ então $a<c$ (transitiva).

Observe que a partir de uma ordem podemos obter uma ordem estrita e vice e versa.

Proposição 1.3. Se $\leq$ é uma ordem sobre $P$, então $<$ dada por, dados $a, b \in P, a<b$ se, e somente se, $a \leq b$ e $a \neq b$, é uma ordem estrita sobre $P$.

Demonstração. Vamos mostrar que $<$ assim definida satisfaz os dois axiomas de ordem estrita.

(1) Seja $a \in A$. Como não é verdade que $a \neq a$, não vale " $a \leq a$ e $a \neq a$ ". Logo, temos que não vale $a<a$.

(2) Sejam $a, b, c \in P$. Suponha que $a<b$ e $b<c$. Mostremos que $a<c$. Note que, como $a<b$ e $b<c$ temos que $(a \leq b$ e $a \neq b)$ e $(b \leq c$ e $b \neq c)$ pela definição de $<$. Pela transitividade de $\leq$, temos que $a \leq c$. Para terminar, vamos mostrar que $a \neq c$. Suponha que $a=c$. Como $a<b$, temos $c<b$. por outro lado, temos que $b<c$. Pela definição de $<$, temos então $b \leq c$ e $c \leq b$. Assim, pela propriedade de antissimetria de $\leq$, temos que $b=c$. Contradição com o fato que $b \neq c$.

Note que, se $<$ é uma ordem estrita sobre $P$, então $\leq$ dada por: dados $a, b \in P, a \leq b$ se, e somente se, $a<b$ ou $a=b$, é uma ordem sobre $P$.

Em determinados conjuntos observamos que existe um elemento que é "menor" que os demais, como por exemplo o 0 em $(\mathbb{N}, \leq)$. Ou um elemento que seja maior que o restante, por exemplo o $1 \mathrm{em}([0,1], \leq)$. 
Definição 1.4. Seja $(P, \leq)$ um conjunto ordenado e seja $A \subset P$ não vazio e $m \in P$. Então:

Dizemos que $m$ é o elemento minimo de $A$ se $m \in A$ e para todo $x \in A$ temos que $m \leq x$.

Dizemos que $m$ é um elemento minimal de $A$ se $m \in A$ e não existe $x \in A$ tal que $x \leq m$.

Dizemos que $m$ é o elemento máximo de $A$ se $m \in A$ e para todo $x \in A$ temos que $x \leq m$.

Dizemos que $m$ é o elemento maximal de $A$ se $m \in A$ e não existe $x \in A$ tal que $m \leq x$.

Dizemos que $m$ é um limitante inferior de A se para todo $x \in A$ temos que $m \leq x$.

Dizemos que $m$ é um limitante superior de $A$ se para todo $x \in A$ temos que $x \leq m$.

Se A possui um limitante superior dizemos que A é limitado superiormente. Se A possui um limitante inferior dizemos que A é limitado inferiormente.

Geralmente o primeiro contato que temos com o conceito de boa ordem é quando estamos estudando a construção dos números naturais. A definição de boa ordem por si é de extrema importância para a teoria de conjuntos. Além disso, em $Z F C$ todo conjunto pode ser bem ordenado ${ }^{1}$.

Definição 1.5. Seja $(P, \leq)$ um conjunto ordenado. Dizemos que $\leq$ é uma boa ordem se, para qualquer $A \subset X$ não vazio, A possui elemento minimo. Dizemos que $(P, \leq)$ é um conjunto bem ordenado se $\leq$ for uma boa ordem.

Observe que, um conjunto bem ordenado $(P, \leq)$ é totalmente ordenado. De fato, sejam $a, b \in P$ e considere o subconjunto $\{a, b\}$, como $P$ é bem ordenado então $\{a, b\}$ possui um elemento mínimo. Logo $a \leq b$ ou $b \leq a$

\footnotetext{
${ }^{1}$ Também conhecido como o Teorema de Zermelo, tal resultado é equivalente ao Axioma da Escolha em $Z F$.
} 
Definição 1.6. Seja $(P, \leq)$ um conjunto ordenado. Dizemos que $D \subset P$ é denso em $P$ se, para todo $p \in P$ existe $d \in D$ tal que $d \leq p$.

Definição 1.7. Seja $(P, \leq)$ um conjunto ordenado. Um subconjunto $C \subset P$ onde $(C, \leq)$ é totalmente ordenado é chamado de cadeia. Dizemos que $A \subset P$ é uma anticadeia se seus elementos são dois a dois incompativeis, isto é, dados a, $b \in A$ distintos, não existe $c \in P$ tal que $c \leq a$ e $c \leq b$.

Com isso podemos enunciar o Lema de Zorn, que assim como o Teorema de Zermelo também é equivalente ao Axioma da Escolha.

Lema 1.8 (Lema de Zorn). Seja $(P, \preceq)$ um conjunto não vazio e ordenado. Se toda cadeia possui um limitante superior então o conjunto $P$ possui elemento maximal.

Definição 1.9. Seja $(P, \leq)$ um conjunto ordenado. Seja $A \subset P . \quad O$ supremo de $A$ $(\sup A)$ é o mínimo, caso exista, do conjunto dos limitantes superiores de $A$, i.e., $\sup A=$ $\min \{x \in P: a \leq x$ para todo $a \in A\}$. O ínfimo de $A$ (inf $A$ ) é o máximo, caso exista, do conjunto dos limitantes inferiores de $A$, i.e., $\inf A=\max \{x \in P: x \leq$ a para todo $a \in A\}$.

\subsection{Ordinais e Cardinais}

O números ordinais, intuitivamente, são uma "generalização" dos números naturais. Para os ordinais também temos um processo de "adição" e isso se dá por meio da Definição 1.13Ordinais e Cardinaisteorema.1.13 onde temos a definição de ordinal sucessor. Muito mais do que isso, os ordinais são os representantes "canônicos" dos conjuntos bem ordenados, tendo em vista que todo conjunto bem ordenado é isomorfo a um único ordinal ( ou seja, existe uma bijeção que preserva a ordem $)^{2}$.

Agora, faremos os pré requisitos necessários para a definição de um número ordinal.

Definição 1.10. Seja $X$ um conjunto. Seja $\in_{X}=\{(x, y) \in X \times X: x \in y\}$.

Definição 1.11. Seja $X$ um conjunto. Dizemos que $X$ é transitivo se para todo $x \in X$ e para todo $y \in x$ tivermos que $y \in X$.

\footnotetext{
${ }^{2}$ Veja [11].
} 
Definição 1.12. Seja $\alpha$ um conjunto. Dizemos que $\alpha$ é um número ordinal se $\alpha$ é transitivo $e\left(\alpha, \in_{\alpha}\right)$ é bem ordenado. Estamos considerando $\alpha:=\{\beta: \beta \in \alpha\}$

Observe que " $\in$ " é uma ordem restrita e a definição de conjuntos bem ordenados é feita para ordens não estrita.

Definição 1.13. Seja a um ordinal. Definimos o ordinal sucessor de a como sendo $\alpha+1=\alpha \cup\{\alpha\}$.

Definição 1.14. Um ordinal $\alpha$ é um ordinal sucessor se $\alpha=\beta+1$, para algum ordinal $\beta$. Caso contrário, $\alpha$ é um ordinal limite.

Definição 1.15. Sejam $\alpha$ e $\beta$ números ordinais. Denotamos:

(1) $\alpha<\beta$ se, e somente se, $\alpha \in \beta$;

(2) $\alpha>\beta$ se, e somente se, $\beta<\alpha$;

(3) $\alpha \leq \beta$ se, e somente se, $\alpha<\beta$ ou $\alpha=\beta$;

(4) $\alpha \geq \beta$ se, e somente se, $\beta<\alpha$ ou $\alpha=\beta$.

Observe que se $X$ é um conjunto de ordinais, então $\bigcup X$ é ordinal ${ }^{3}$.

Com isso podemos caracterizar o supremo e o ínfimo de um conjunto de ordinais.

Proposição 1.16. Se $X$ é um conjunto de ordinais, então o sup $X=\bigcup X$ (supremo de $X$ ) e se $X \neq \emptyset$ temos que $\inf X=\bigcap X$ (infimo de $X$ ).

De maneira parecida com os números naturais, temos o princípio de indução para números ordinais. Somente enunciaremos o teorema de maneira informal, para acompanhar os detalhes de sua demonstração, veja [8].

Teorema 1.17 (Indução Transfinita). Seja X uma coleção de ordinais e assuma que:

(1) $0 \in X$;

(2) se $\alpha \in X$, então $\alpha+1 \in X$;

\footnotetext{
${ }^{3}$ Veja [8] ou [11]
} 
(3) se $\alpha$ é um ordinal limite $e \beta \in X$ para todo $\beta<\alpha$, então $\alpha \in X$.

Então $X$ é a coleção de todos os ordinais ${ }^{4}$.

Vamos definir o que é um número cardinal.

Definição 1.18. Seja $\gamma$ um ordinal. Então $\gamma$ é um número cardinal se não existe bijeção $f: \gamma \rightarrow \beta \operatorname{com} \beta<\gamma$.

Definição 1.19. Seja $\kappa$ um cardinal. Definimos o cardinal sucessor de $\kappa$ como sendo $\kappa^{+}=\min \{\beta: \beta$ é cardinal e $\kappa<\beta\}$.

Dado um ordinal $\alpha$ como saber em quantos passos ele é atingido? Seguindo essa idéia intuitiva definiremos o que é a cofinalidade de um ordinal. Mas antes disso:

Definição 1.20. Seja $f: \beta \rightarrow \alpha$. Dizemos que $f$ é ilimitada se para cada $\zeta<\alpha$, existe $\xi<\beta$ tal que $\zeta<f(\xi)<\alpha$.

Definição 1.21. Seja $\alpha$ um ordinal. A cofinalidade de $\alpha(c f(\alpha))$ é o menor ordinal $\beta$, tal que existe uma função $f: \beta \rightarrow \alpha$ crescente e ilimitada em $\alpha$.

A cofinalidade só será interessante nos casos em que $\alpha$ for ordinal limite. Observe que a função identidade $f: \alpha \rightarrow \alpha$, para $\alpha$, é crescente e ilimitada. Por isso $c f(\alpha) \leq \alpha$.

Note não é necessário que $f: \beta \rightarrow \alpha$ seja crescente, pois sempre podemos definir uma função auxiliar $\tilde{f}(\xi)=\sup _{\eta<\xi} f(\eta)$ que será crescente e ilimitada.

Proposição 1.22. Seja $\alpha$ um ordinal e $c f(\alpha)=\beta$. Então $c f(\beta)=\beta$.

Demonstração. Seja $\gamma=c f(\beta)$, logo, pela observação acima $\gamma \leq \beta$.

Vamos mostrar que $\beta \leq \gamma$.

Temos que:

- $\beta$ é o menor ordinal tal que exista $f: \beta \rightarrow \alpha$ crescente e ilimitada.

- $\gamma$ é o menor ordinal tal que exista $g: \gamma \rightarrow \beta$ crescente e ilimitada.

Seja $h=f o g: \gamma \rightarrow \alpha$.

\footnotetext{
${ }^{4}$ Neste caso $X$ não é formalmente um conjunto, $X$ seria a classe de todos os ordinais. Veja [8].
} 
(1) $h$ é crescente. Sejam $x<y<\gamma$, como $g$ é crescente segue que $g(x)<g(y)$ e como $f$ é crescente temos que $f(g(x))<f(g(y))$.

(2) $h$ é ilimitada. Seja $\zeta<\alpha$, por $f$ ser ilimitada existe $\xi<\beta$ tal que $\zeta<f(\xi)<\alpha$. Por $g$ ser ilimitada implica que existe $\eta<\gamma$ tal que $\xi<g(\eta)<\gamma$. Como $f$ é crecente temos que $\zeta<f(\xi)<f(g(\eta))<\alpha$. Logo, dado $\zeta<\alpha$ implica que existe $\eta<\gamma$ tal que $\zeta<h(\eta)<\alpha$.

Como $\beta$ é o menor ordinal tal que existe $f: \beta \rightarrow \alpha$ crescente e ilimitada segue que $\beta \leq \gamma$. Portanto $\gamma=\beta$.

Definição 1.23. Seja $\kappa$ um cardinal. Dizemos que $\kappa$ é um cardinal regular se $c f(\kappa)=$ $\kappa$.

\section{3 Árvores}

Podemos ver uma árvore como um exemplo de conjunto parcialmente ordenado. A vantagem de se trabalhar com árvores é o seu recurso visual, pois podemos imaginar que começamos com um ponto (chamado de raíz ${ }^{5}$ ) e o restante dos pontos "sobem" em várias ramificações, tendo em mente que essas ramificações não se cruzam.

Definição 1.24. Seja $(T, \leq)$ um conjunto ordenado. Dizemos que $(T, \leq)$ é uma árvore se, para qualquer $t \in T$, o conjunto $\{s \in T: s \leq t\}$ é bem ordenado por $\leq$.

Definição 1.25. Dizemos que $R \subset T$ é um ramo se for uma cadeia maximal. Dizemos que $r \in T$ é raiz de $T$ se $r=\min T$ (note que $T$ pode não ter raiz). Caso $T$ não tenha uma raíz podemos definir em $T$ um elemento $r$ menor que todos os minimais de $T$.

Proposição 1.26. Se $(T, \leq)$ é uma árvore e $p, q \in T$ são incomparáveis, então não existe $t \in T$ tal que $p, q \leq t$.

Demonstração. Suponha que exista $t \in T$ tal que $p, q \leq t$. Então como $T$ é árvore, por hipótese temos que o conjunto $A=\{s \in T: s \leq t\}$ é bem ordenado. Note que

\footnotetext{
${ }^{5} \mathrm{Na}$ verdade, pode não existir raíz.
} 
$p, q \in A$. Daí o subconjunto $\{p, q\}$ possui um menor elemento e disso segue que $p$ e $q$ são comparáveis o que é uma contradição.

Considere o seguinte conjunto:

Definição 1.27. Seja $\omega^{<\omega}=\bigcup_{n \in \omega} \omega^{n}$. Onde $\omega^{n}=\{f \mid f: n=\{0, \ldots, n-1\} \rightarrow \omega\}$.

Com isso, temos um exemplo de árvore.

Proposição 1.28. $\left(\omega^{<\omega}, \leq\right)$ é uma árvore. Onde $f \leq g$ se, e somente se, $f \subset g$ (g estende $f)^{6}$.

Demonstração. Seja $f \in \omega^{<\omega}$ e considere o conjunto $A_{f}=\left\{s \in \omega^{<\omega}: s \leq f\right\}$. Note que $A_{f} \neq \emptyset$. Seja $B \subset A_{f} \operatorname{com} B \neq \emptyset$. Como $B$ é não vazio, basta tomar a restrição de $f$ com menor domínio que pertença a $B$.

Note que $\omega^{<\omega}$ é enumerável. Para isso, basta notar que $\omega^{<\omega}=\bigcup_{n \in \omega} \omega^{n}$ e, por sua vez, que cada $\omega^{n}=\{f \mid f: n \rightarrow \omega\}$ é enumerável.

Proposição 1.29. Se $R \subset \omega^{<\omega}$ é um ramo, então $\bigcup R$ é uma função de $\omega$ em $\omega$.

Demonstração.

(1) $U R$ é uma função. De fato, sejam $(a, b),(a, c) \in \bigcup R$. Então existem $s, t \in R$ tal que $(a, b) \in s$ e $(a, c) \in t$. Sem perda de generalidade, suponha que $s \subset t^{7}$ então $(a, b) \in t$ e, como $t$ é função, segue que $(a, b)=(a, c)$.

(2) $\operatorname{dom} \bigcup R=\omega$. De fato, suponha que não. Seja $a \in \omega$ tal que $a$ seja o menor elemento que não esteja no domínio da função $\bigcup R$. Note que toda $f \in R$ é tal que $\operatorname{dom} f \subset a$. Tome $f: a \rightarrow \omega$ tal que $f \in R$. Note que tal $f$ existe, pois $R$ é um ramo. Defina $g: a+1 \rightarrow \omega$ tal que $g(n)=f(n)$ se $n<a$ e $g(n)=7$ se $n=a$, por exemplo. Daí para todo $t \in R, t \subset g$ e $g \notin R$. Assim, $R \cup\{g\}$ é uma cadeia que contém $R$ o que é uma contradição com a maximalidade de $R$. Portanto, o domínio de $\bigcup R$ é todo o $\omega$.

\footnotetext{
${ }^{6}$ Para essa proposição e resultados futuros pensamos em funções como conjuntos de pares ordenados.

${ }^{7} R$ é um conjunto totalmente ordenado por $\subset$.
} 
Portanto $\bigcup_{s \in R} s$ é uma função de $\omega$ em $\omega$ para cada $R$ ramo. Além disso, tomando-se ramos diferentes, temos funções diferentes.

Proposição 1.30. Considere $\mathfrak{R}$ o conjunto dos ramos de $\omega^{<\omega}$. Então $F: \mathfrak{R} \rightarrow \omega^{\omega}$ dada por $F(R)=\bigcup_{s \in R} s$ é uma bijeção.

Demonstração. A função $F$ está bem definida em vitude da Proposição 1.29Árvoresteorema.1.29.

Para mostrar que $F$ é sobrejetora, tome $s \in \omega^{\omega}$. Para cada $n \in \omega$, seja $s_{n}: n \rightarrow \omega$ dada por $s_{n}=s \mid n$. Seja $R=\left\{s_{n}: n<\omega\right\}$. Observe que $R$ é um ramo, pois todos os seus elementos são comparáveis e $R$ é maximal. Note que $F(R)=s$.

Sejam $R_{1}$ e $R_{2}$ dois ramos distintos de $\mathfrak{R}$. Então existe $r_{1} \in R_{1}$ e $r_{2} \in R_{2}$ que não são comparáveis, caso contrário, conseguimos $R_{1} \subset R_{2}$ e $R_{2} \subset R_{1}^{8}$ contradizendo a maximamilidade. Portanto $F$ é injetora.

Definição 1.31. Dado $t \in T$, dizemos que $s \in T$ é um sucessor de $t$ se $t<s$, dado $r \in T$ tal que $t \leq r \leq s$, então $r=t$ ou $r=s$.

Proposição 1.32. Se $(T, \leq)$ é uma árvore infinita com uma raíz tal que o conjunto sucessor de cada elemento é finito, então $T$ tem um ramo infinito.

Demonstração. Seja $r$ a raiz de $T$ e para cada $p \in T$ defina os seguintes conjuntos:

$$
\begin{gathered}
\operatorname{suc}(p)=\{t \in T: t \text { é sucessor de } p\} \\
A_{p}=\{t \in T: p<t\}
\end{gathered}
$$

Vamos fazer a prova por indução. Chame $p_{0}=r$. Por hipótese $\operatorname{suc}\left(p_{0}\right)$ é finito, então escolha $p_{1} \in \operatorname{suc}\left(p_{0}\right)$ tal que $A_{p_{1}}$ seja infinito. Note que isso é possível, pois, $T$ é infinita. Suponha feita a mesma construção até $p_{n}$, então escolha $p_{n+1} \in \operatorname{suc}\left(p_{n}\right)$ tal que $A_{p_{n+1}}$ seja infinito. Então $R=\left\{p_{n}: n \in \omega\right\}$ é um ramo infinito.

Definição 1.33. Dada uma árvore $(T, \leq)$, dizemos que $A \subset T$ é uma anticadeia no sentido de árvore se para todo $a, b \in A$ distintos, temos que a e b são incomparáveis.

\footnotetext{
$R_{2}$.

${ }^{8}$ pois $R_{1} \cup R_{2}$ seria uma cadeia. Como $R_{1}, R_{2} \subset R_{1} \cup R_{2}$ e ambos são ramos, segue que $R_{1}=R_{1} \cup R_{2}=$
} 
Definição 1.34. Seja $(T, \leq)$ uma árvore. Chamamos de altura de $t \in T$ o (único) ordinal a que $\{s \in T: s<t\}$ é isomorfo (notação $h(t)$ ). Dado um ordinal $\alpha$, denotamos por Lev $\operatorname{Le}_{\alpha}(T)$ o conjunto $\{t \in T: h(t)=\alpha\}$ e chamamos de nível $\alpha$ da árvore. Chamamos de altura de $T$ (notação $h(T)$ ) o primeiro ordinal $\alpha$ tal que $\operatorname{Lev}_{\alpha}(T)=\emptyset$.

Proposição 1.35. $\omega^{<\omega}$ é uma árvore de altura $\omega$ e que possui ramos enumeráveis.

Demonstração. Pela Proposição 1.28Árvoresteorema.1.28 temos que $\omega^{<\omega}$ é uma árvore. Mostremos que $h\left(\omega^{<\omega}\right)=\omega$. Para isso, suponha que exista $t \in \omega^{<\omega}$ tal que $h(t)=\omega$, ou seja, o conjunto $\left\{s \in \omega^{<\omega}: s \leq t\right\}$ é isomorfo a $\omega$. Porém, $|t|=n$, para algum $n \in \omega$, e com isso, $\left\{s \in \omega^{<\omega}: s \leq t\right\}$ é finito. Portanto, $\left\{t \in \omega^{<\omega}: h(t)=\omega\right\}=\emptyset$.

Agora, suponha que exista $k<\omega$ tal que $\left\{t \in \omega^{<\omega}: h(t)=k\right\}=\emptyset$. Considere $s=\{(0,0), \ldots,(k-1,0)\}$ e note que $h(s)=k$. Portanto, $\omega$ é o primeiro ordinal tal que $\operatorname{Lev}_{\omega}\left(\omega^{<\omega}\right)=\emptyset$.

Finalmente, note que $R=\{\{(0,0)\},\{(0,0),(1,0)\},\{(0,0),(1,0),(2,0)\}, \ldots\}$ é um ramo e que $|R|=\omega$.

Proposição 1.36. Dada uma árvore $(T, \leq)$. Então $\preceq d a d a$ por $a \preceq b$ se $b \leq a$ é uma ordem. Vamos chamar esta de ordem reversa.

Demonstração.

(1) $a \preceq a$, pois $a \leq a$ para todo $a \in T$;

(2) $a \preceq b$ e $b \preceq a$ implica que $a=b$, pois $b \leq a$ e $a \leq b$ implicam que $a=b$.

(3) $a \preceq b$ e $b \preceq c$ implica que $a \preceq c$, pois $b \leq a$ e $c \leq b$ implicam que $c \leq a$.

Proposição 1.37. Seja $(T, \leq)$ uma árvore e seja $A \subset T$. Então $A$ é anticadeia no sentido de árvore se, e somente se, A é anticadeia no sentido de ordem, mas usando a ordem reversa. 
Demonstração. Seja $A$ uma anticadeia no sentido de árvore e sejam $a, b \in A$. Então pela Proposição 1.26Árvoresteorema.1.26 não existe $t \in T$ tal que $a, b \leq t$. Em termos de ordem reversa, isso implica que não existe $t \in T$ tal que $t \preceq a, b$. Portanto $A$ é uma anticadeia no sentido de ordem reversa. Reciprocamente, seja $A$ uma anticadeia no sentido de ordem reversa. Suponha que $A$ não seja uma anticadeia no sentido de árvore. Então existe $a, b \in A$ tal que $a \leq b$, então $a, b \leq b$ que implica $b \preceq a, b$ o que é um absurdo.

Corolário 1.38. Seja $(T, \leq)$ uma árvore. Dados $a, b \in T$ distintos. Então $a, b \in(T, \leq)$ são incomparáveis se, e somente se, $a, b \in(T, \preceq)$ são incompativeis.

Definição 1.39. Seja $(T, \leq)$ uma árvore. Uma sub-árvore de $T$ é um subconjunto $T^{\prime} \subset T$ com a ordem induzida tal que para todo $x \in T^{\prime}$ e para todo $y \in T$ com $y \leq x$ temos que $y \in T^{\prime}$.

Definição 1.40. Uma árvore de Aronszajn é uma árvore de altura $\omega_{1}$ onde todos os seus ramos são enumeráveis e todo nível é enumerável.

Assumindo $Z F C$, existe uma árvore de Aronszajn.

Teorema 1.41. Existe uma árvore de Aronszajn.

Demonstração. Seja $T=\left\{s \in \omega^{<\omega_{1}}: s\right.$ é injetora $\}$ ordenado pela inclusão, lembrando que $\omega^{<\omega_{1}}=\bigcup_{\alpha<\omega_{1}} \omega^{\alpha}$. Observe que:

- $\omega^{<\omega_{1}}$ é árvore. Seja $s \in T$ e seja $\{t \in T: t \leq s\}$. Tome $A \subset\{t \in T: t \leq s\}$ tal que $A \neq \emptyset$. Seja $B=\{\operatorname{dom} t: t \in A\}$ e note que $B \neq \emptyset$ e $B \subset \omega_{1}$. Como $\omega_{1}$ é bem ordenado, segue que existe o elemento mínimo de $B$. Seja tal elemento $\kappa$. Agora, observe que $\kappa$ é o domínio de $t^{\prime}$ onde $t^{\prime}=\min A$.

- $h(T)=\omega_{1}$. Observe que $\operatorname{Lev}_{\alpha}(T)$ possui todas as funções injetoras de $\omega^{\alpha}$ para $\alpha<\omega_{1}$. Logo $\operatorname{Lev}_{\alpha}(T) \neq \emptyset$ para $\alpha<\omega_{1}$. Agora, como não existe uma $f: \omega_{1} \rightarrow \omega$ injetora temos que $\operatorname{Lev}_{\omega_{1}}(T)=\emptyset$.

- $T$ somente possui ramos enumeráveis. Suponha que exista uma ramo não enumerável. Denotemos por $C$ tal ramo. Vamos mostrar que $\bigcup C: \omega_{1} \rightarrow \omega$ é uma 
função injetora. Facilmente vemos que $\bigcup C$ é uma função injetora (basta proceder de maneira análoga a que foi feito na Proposição 1.29Árvoresteorema.1.29). Agora, para ver que $\operatorname{dom} \bigcup C=\omega_{1}$, temos que $\operatorname{dom} \bigcup C \subset \omega_{1}$ e, caso a inclusão contrária não valesse teríamos que $C$ seria enumerável.

Note $T$ não é Aronszajn, pois existem níveis não enumeráveis. Para ver isso, basta notar que $\operatorname{Lev}_{\alpha}(T)$ é não enumerável para $\omega \leq \alpha<\omega_{1}$, pois $\left|\operatorname{Lev}_{\alpha}(T)\right|=\omega^{\alpha} \geq \omega^{\omega} \geq 2^{\omega} \geq$ $\omega_{1}$.

Porém, vamos definir uma sub-árvore de $T$ que seja Aronszajn.

Se $s, t \in \omega^{\alpha}$, defina $s \sim t$ se, e somente se, $\{\xi<\alpha: s(\xi) \neq t(\xi)\}$ é finito. Vamos encontrar $s_{\alpha}$ para $\alpha<\omega_{1}$ tal que:

(i) $s_{\alpha} \in \omega^{\alpha}$ e $s_{\alpha}$ é injetora,

(ii) se $\alpha<\beta$ então $s_{\alpha} \sim s_{\beta} \mid \alpha$, e

(iii) $\omega \backslash \operatorname{Im}\left(s_{\alpha}\right)$ é infinito.

Assumindo que tais $s_{\alpha}$ 's possam ser encontrados, seja

$$
T^{*}=\bigcup_{\alpha<\omega_{1}}\left\{t \in \operatorname{Lev}_{\alpha}(T): t \sim s_{\alpha}\right\}
$$

- $T^{*}$ é uma sub-árvore de $T$. Seja $t \in T$ e seja $s \in T^{*}$ tal que $t \leq s$. Então existe $\alpha<\omega_{1}$ tal que $s \sim s_{\alpha}$ e existe $\beta<\alpha$ tal que $h(t)=\beta$. Por $(i i)$ temos que $s_{\beta}=s_{\alpha} \mid \beta$ e $t \sim s_{\alpha} \mid \beta$ então $t \sim s_{\beta}$. Portanto $t \in T^{*}$.

- Por $(i)$, temos que $s_{\alpha} \in T^{*}$. Note também que $h^{*}(t)=h(t)$ para todo $t \in T^{*}$ (onde $h^{*}(t)$ é a altura de $t$ em relação a árvore $\left.T^{*}\right)$ pelo fato de $T^{*}$ ser uma sub-árvore de $T$. Com isso temos que $\operatorname{Lev}_{\alpha}\left(T^{*}\right) \subset \operatorname{Lev}_{\alpha}(T)$. Em outras palavras, $\operatorname{Lev}_{\alpha}\left(T^{*}\right)=$ $\left\{t \in \operatorname{Lev}_{\alpha}(T): t \sim s_{\alpha}\right\}$.

- $\left\{t \in \operatorname{Lev}_{\alpha}(T): t \sim s_{\alpha}\right\}$ é enumerável. Para ver isso, seja $\mathcal{B}=\{B \subset \alpha: B$ é finito $\}$. Para cada $B \in \mathcal{B}$ defina $T_{B}=\left\{t \in \operatorname{Lev}_{\alpha}(T): t(\xi) \neq s_{\alpha}(\xi)\right.$ para $\xi \in B$ e $t(\xi)=s_{\alpha}(\xi)$ para $\xi \notin B\}$. Note que $T_{B}$ é enumerável, pois o conjunto das funções $\{f \mid f: B \rightarrow \omega\}$ 
é enumerável. Temos também que $\bigcup_{B \in \mathcal{B}} T_{B}$ é enumerável (pois $\mathcal{B}$ é enumerável). E, como $\left\{t \in \operatorname{Lev}_{\alpha}(T): t \sim s_{\alpha}\right\} \subset \bigcup_{B \in \mathcal{B}} T_{B}$, segue que $\left\{t \in \operatorname{Lev}_{\alpha}(T): t \sim s_{\alpha}\right\}$ é enumerável.

Portanto, $T^{*}$ é uma árvore de Aronszajn.

Agora, vamos construir os $s_{\alpha}$ 's por indução. Suponha que $s_{\alpha}$ esteja definido para $\alpha<\gamma$.

- Se $\gamma$ é sucessor (i.e., $\gamma=\beta+1$ para algum $\beta$ ). Tome qualquer $n \in \omega \backslash \operatorname{Im}\left(s_{\beta}\right)$ e seja $s_{\gamma}=s_{\beta} \cup\{(\gamma, n)\}$. Observe que $s_{\gamma} \operatorname{satisfaz}(i)$ e $(i i)$ e $\omega \backslash \operatorname{Im}\left(s_{\gamma}\right)=\left(\omega \backslash \operatorname{Im}\left(s_{\beta}\right)\right) \backslash\{n\}$ que é infinito e portanto também satisfaz (iii).

- Se $\gamma$ for limite. Seja $\left(\alpha_{n}\right)_{n \in \omega}$ uma sequência crescente e cofinal em $\gamma$. A primeira vista poderíamos pensar em tomar $s_{\gamma}=\bigcup_{n \in \omega} s_{\alpha_{n}}$, porém não há garantias que sequer essa união seja uma função (já que $s_{\alpha_{n}} \nsubseteq s_{\alpha_{n}}$ ). Para contornar esse "problema" construiremos funções injetoras auxiliares $t_{0} \leq t_{1} \leq \cdots$ tais que $t_{n} \sim s_{\alpha_{n}}$ e $t_{n+1} \mid \alpha_{n}=t_{n}$. Seja $t_{0}=s_{\alpha_{0}}$. Observe que $A_{1}=\left\{\kappa \in \alpha_{0}: t_{0}(\kappa) \neq s_{\alpha_{1}}(\kappa)\right\}$ é finito (a imagem de $s_{\alpha_{0}}$ nesses pontos podem coincidir com a imagem de $s_{\alpha_{1}}$ para pontos maiores que $\alpha_{0}$ ), ou seja, $A_{1}=\left\{n_{0}, \ldots, n_{k}\right\}$ (sem perda de generalidade, suponha $\left.n_{0}<\cdots<n_{k}\right)$. Defina $t_{1}$ como:

$$
t_{1}(\kappa)= \begin{cases}s_{\alpha_{1}}(\kappa) \quad & \text { se } \alpha_{0} \leq \kappa, \\ s_{\alpha_{0}}(\kappa) \quad & \text { se } n<\alpha_{0} \text { e } \kappa \notin A_{1}, \\ a_{0} & \text { se } \kappa=n_{0}, \text { onde } a_{0} \in\left[\omega \backslash \operatorname{Im}\left(t_{0}\right)\right] \backslash\left\{t_{0}\left(n_{0}\right), \ldots, t_{0}\left(n_{k}\right)\right\} \\ a_{1} & \text { se } \kappa=n_{1}, \text { onde } a_{1} \in\left[\omega \backslash \operatorname{Im}\left(t_{0}\right)\right] \backslash\left\{t_{0}\left(n_{0}\right), \ldots, t_{0}\left(n_{k}\right), a_{0}\right\} \\ \vdots & \text { se } \kappa=n_{k}, \text { onde } a_{k} \in\left[\omega \backslash \operatorname{Im}\left(t_{0}\right)\right] \backslash\left\{t_{0}\left(n_{0}\right), \ldots, t_{0}\left(n_{k}\right), a_{0}, \ldots, a_{k-1}\right\} \\ a_{k} & \end{cases}
$$

observe que $t_{1}$ é injetora e $t_{1} \sim s_{\alpha_{1}}$, pois $t_{1} \mid \alpha_{0} \sim s_{\alpha_{0}}$. Além disso $\omega \backslash \operatorname{Im}\left(t_{1}\right)$ é infinito. Indutivamente defina $t_{n}: \alpha_{n} \rightarrow \omega$ tal que $t_{n}$ seja injetora, $t_{n} \sim s_{\alpha_{n}}$, e $t_{n+1} \mid \alpha_{n}=t_{n}$ 
Seja $t=\bigcup_{n<\omega} t_{n}$ então $t \in \omega^{\gamma}$ e $t$ é injetora. Se colocarmos que $s_{\gamma}=t$, então $(i)$ e (ii) valem, mas (iii) pode falhar. Para consertar isso, defina $s_{\gamma}\left(\alpha_{n}\right)=t\left(\alpha_{2 n}\right)$ e $s_{\gamma}(\xi)=t(\xi)$ para $\xi \notin\left\{\alpha_{n}: n \in \omega\right\}$. Então

$$
\left\{t\left(\alpha_{2 n+1}\right): n \in \omega\right\} \subset\left(\omega \backslash \operatorname{Im}\left(s_{\gamma}\right)\right)
$$

Portanto $\omega \backslash \operatorname{Im}\left(s_{\gamma}\right)$ é infinito.

Definição 1.42. Uma árvore bem podada $(T, \leq)$ é uma árvore tal que $\left|\operatorname{Lev}_{0}(T)\right|=1$ (ou seja, tem raiz) e, para todo $x \in T$ e para todo $\alpha$ tal que $h(x)<\alpha<h(T)$ existe $y \in \operatorname{Lev}_{\alpha}(T)$ tal que $x<y$.

Proposição 1.43. Seja $(T, \leq)$ é uma árvore de altura $\omega_{1}$ tal que para todo $\alpha<\omega_{1}$ temos $\left|\operatorname{Lev}_{\alpha}(T)\right|<\omega_{1}$. Então $T$ possui uma sub-árvore $T^{\prime}$ bem podada de altura $\omega_{1}$.

Demonstração. Seja $T^{\prime}=\left\{x \in T:|\{z \in T: x<z\}|=\omega_{1}\right\}$.

(1) $T^{\prime}$ é uma sub-árvore de $T$.

Sejam $x \in T^{\prime}$ e $y \in T$ com $y<x$, então $\{z \in T: y<z\} \supset\{z \in T: x<z\} \operatorname{logo}$ $|\{z \in T: y<z\}| \geq|\{z \in T: x<z\}|$. Como $|\{z \in T: x<z\}|=\omega_{1}$, então $|\{z \in T: y<z\}| \geq \omega_{1}$. Portanto $y \in T^{\prime}$.

(2) $T^{\prime}$ satisfaz é bem podada.

Sejam $x \in T^{\prime}$ fixo e $\alpha$ tal que $h(x)<\alpha<\omega_{1}$. Seja $Y=\left\{y \in \operatorname{Lev}_{\alpha}(T): x<y\right\}$. Pela definição de $T^{\prime}$ e pelo fato de que cada $\left|\operatorname{Lev}_{\beta}(T)\right|<\omega_{1}$ e $\mid\{z \in T: x<z$ e $\alpha<h(z)\} \mid=\omega_{1}$. De fato, seja $\beta=h(x)$, observe que, para cada $\beta<\gamma<\alpha$ o conjunto $\left|\left\{y \in \operatorname{Lev}_{\gamma}(T): x<y\right\}\right|<\omega_{1}$ e $\operatorname{assim}\left|\bigcup_{\beta<\gamma<\alpha}\left\{y \in \operatorname{Lev}_{\gamma}(T): x<y\right\}\right|<\omega_{1}$. Como $|\{z \in T: x<z\}|=\omega_{1} \mathrm{e}$

$$
\{z \in T: x<z\}=\bigcup_{\beta<\gamma<\alpha}\left\{y \in \operatorname{Lev}_{\gamma}(T): x<y\right\} \cup\{z \in T: x<z, \alpha<h(z)\}
$$


segue que $\mid\{z \in T: x<z$ e $\alpha<h(z)\} \mid=\omega_{1}$. Como $|Y|<\omega_{1}$, existe um $y \in Y$, tal que $|\{z \in T: z>y\}|=\omega_{1}$.

Portanto $y \in T^{\prime}$.

Definição 1.44. Dizemos que uma árvore $(T, \leq)$ é uma árvore de Suslin se $T$ tem altura $\omega_{1}$ e não posui anticadeias e ramos não enumeráveis.

No Capítulo 5A Hipótese de Suslinchapter.5 estudaremos esse tipo de árvore com mais detalhes. Como consequência da Proposição anterior temos uma resultado que será útil futuramente.

Corolário 1.45. Se existe uma árvore de Suslin então existe $(T, \leq)$ que é árvore de Suslin bem podada.

\section{4 Álgebra de Boole}

A álgebra booleana foi criada como uma tentativa de utilizar técnicas algébricas para lidar com expressões lógicas do cálculo proposicional.

Veremos que, assim como árvores, uma álgebra boolena é um exemplo de um conjunto parcialmente ordenado.

Definição 1.46. $(A,+, \cdot,-, 0,1)$ chama-se álgebra de Boole se

- "十,." são operações binárias em A;

- "-" é uma operação unária em A;

- $0,1 \in A$.

E para todo $x, y, z \in A$ valem:
(A1) $x+y=y+x$
$\left(A 1^{\prime}\right) x \cdot y=y \cdot x$
$(A 2) x+(y+z)=(x+y)+z$
$\left(A 2^{\prime}\right) x \cdot(y \cdot z)=(x \cdot y) \cdot z$
$(A 3) x \cdot(y+z)=(x \cdot y)+(x \cdot z)$
$\left(A 3^{\prime}\right) x+(y \cdot z)=(x+y) \cdot(x+z)$
(A4) $x+(x \cdot y)=x$
$\left(A 4^{\prime}\right) x \cdot(x+y)=x$
$(A 5) x+(-x)=1$
$\left(A 5^{\prime}\right) x \cdot(-x)=0$ 
Vejamos o seguinte exemplo de álgebra de Boole:

Seja $(\{0,1\},+, \cdot,-, 0,1)$ onde as operações de $+\mathrm{e} \cdot$ são dadas pelas tabelas:

\begin{tabular}{|c|c|c|}
\hline+ & 0 & 1 \\
\hline 0 & 0 & 1 \\
\hline 1 & 1 & 1 \\
\hline
\end{tabular}

\begin{tabular}{|l|l|l|}
\hline$\cdot$ & 0 & 1 \\
\hline 0 & 0 & 0 \\
\hline 1 & 0 & 1 \\
\hline
\end{tabular}

Além disso, temos que $-0=1$ e $-1=0$.

Lema 1.47. Seja A uma álgebra de Boole. Dados $x, y, z \in A$, valem:

(1) $x+x=x, x \cdot x=x$;

(2) $x \cdot 0=0, x+1=1$;

(3) $x \cdot 1=x, x+0=x$;

(4) $-0=1,-1=0$.

Demonstração. Demostraremos apenas o primeiro resultado de cada ítem. Os segundos são análogos.

(1) $x \stackrel{(A 4)}{=} x+x x \stackrel{\left(A 3^{\prime}\right)}{=}(x+x) \cdot(x+x) \stackrel{(A 3)}{=}(x+x) x+(x+x) x \stackrel{\left(A 1^{\prime}\right)}{=}$ $x(x+x)+x(x+x) \stackrel{\left(A 4^{\prime}\right)}{=} x+x ;$

(2) $x \cdot 0 \stackrel{\left(A 5^{\prime}\right)}{=} x \cdot(x \cdot(-x)) \stackrel{\left(A 2^{\prime}\right)}{=}(x \cdot x)(-x) \stackrel{(1)}{=} x \cdot(-x) \stackrel{\left(A 5^{\prime}\right)}{=} 0$;

(3) $x \cdot 1 \stackrel{(2)}{=} x(x+1) \stackrel{\left(A 4^{\prime}\right)}{=} x ;$

(4) $-0 \stackrel{(3),(A 1)}{=} 0+(-0) \stackrel{(A 5)}{=} 1$.

Corolário 1.48. Existe apenas uma única álgebra de Boole com dois elementos.

Demonstração. Se $0 \neq 1$, temos todas as operações definidas acima. Vamos agora mostrar que se $0=1$ então a álgebra só tem um elemento. Suponha que não, então seja $a \neq 0$. Pelo lema anterior,temos que $a \cdot 1=a$ e $a \cdot 0=0$ e então $a=0=1$. 
Definição 1.49. Seja A uma Álgebra de Boole. Definimos $a \leq b$ se $a \cdot b=a$.

Proposição 1.50. s é uma ordem parcial.

Demonstração. Sejam $x, y$ e $z \in A$.

- $x \leq y$ e $y \leq z$ então $x \leq z:$ Temos $x=x \cdot y$ e $y=y \cdot z$. Então $x=x \cdot y=x \cdot(y \cdot z)=$ $(x \cdot y) \cdot z=x \cdot z$, isto é, $x \leq z$.

- $x \leq x$ : Consequencia imediata do Lema 1.47lgebradeBooleteorema.1.47.

- $x \leq y$ e $y \leq x$ então $x=y: x=x \cdot y=y \cdot x=y$.

Observe que $(\wp(X), \cup, \cap, \backslash, \emptyset, X)$ é uma álgebra de Boole. Nesse caso $A \leq B$ se $A \cap B=A$, i.e., $A \subset B$.

\subsection{Filtros e Ultrafiltros}

Podemos pensar em um filtro com sendo o dual de um ideal em álgebra. Enquanto os ideias são conjuntos que "fecham para baixo" (pela operação em questão) os filtros são conjuntos "fechados para cima". No Capítulo 80 espaço de Stone de uma álgebra de Boolechapter.8 será feita uma demostração do Teorema de Tychonoff usando fortemente tal conceito. Além disso, o filtro está presente no enunciado do Axioma de Martin, que veremos no Capítulo 3Dois Axiomas Extras à ZFCchapter.3.

Definição 1.51. Seja $(P, \leq)$ um conjunto ordenando não vazio. Dizemos que $F \subset P e ́$ um filtro sobre $P$ se:

(1) $F \neq \emptyset$ e $F \neq P$;

(2) Se $a, b \in F$, existe $c \in F$ tal que $c \leq a, b$;

(3) Se $p \in P$ e $a \in F$ são tais que $a \leq p$, então $p \in F$.

Proposição 1.52. Seja $X$ um conjunto não vazio e uma coleção $F$ de subconjuntos de $X$ tal que: 
(1) $X \in F$ e $\notin F$;

(2) $S e A \in F$ e $B \in F$, então $A \cap B \in F$;

(3) Se $A, B \subset X A \in F$ e $A \subset B$ então $B \in F$.

Então F é um filtro.

Demonstração. Considere a ordem parcial da inclusão $(\wp(X), \subset) . F \neq \emptyset$, pois $\emptyset \notin F$, os demais ítens seguem da definição.

Vejamos os seguintes exemplos de filtros:

Seja $X$ um conjunto não vazio.

(1) Filtro trivial: $F=\{X\}$;

(2) Seja $X_{0}$ um subconjunto não vazio de $X$. Seja $F=\left\{A \subset X: X_{0} \subset A\right\}$;

(3) Sejam $X$ um espaço topológico e $x \in X$ um ponto qualquer. Então $\{V \subset X: V$ é vizinhança de $x\}$ é um filtro sobre $X$.

Quando falamos de vizinhança não se trata de vizinhança aberta, mas de um subconjunto de $X$ que contenha um aberto contendo o ponto $x$. Se considerarmos somente vizinhanças abertas a terceira condição da definição de filtro pode não valer.

Definição 1.53. Seja $(P, \leq)$ um conjunto ordenado. $P$ possui a propriedade da intersecção finita (p.i.f.) se para qualquer subconjunto finito $G \subset P$ existir $p_{G} \in P$ tal que $p_{G} \leq g$, para todo $g \in G$.

Observação 1.54. Dado $(P, \leq)$ um conjunto parcialmente ordenado e um subconjunto com p.i.f. $E \subset P$ que satisfaça a seguinte propriedade: para todos $a, b \in E$ existe $c \in E$ tal que $c \leq a, b$. Com isso podemos gerar um filtro que contenha $E$, basta definir $F=$ $\{r \in P: p \leq r$ para algum $p \in E\}$. Observe que $F$ satisfaz as condições da Definição 1.51Filtros e Ultrafiltrosteorema.1.51.

Note que se $F$ é um filtro em $X$ então $F$ possui p.i.f. pelo segundo ítem da Definição 1.51Filtros e Ultrafiltrosteorema.1.51. 
Definição 1.55. Dizemos que F é um ultrafiltro se $F$ é filtro e se, para qualquer G filtro tal que $G \supset F$ temos $G=F$.

Agora, um exemplo de ultrafiltro:

Seja $X$ um conjunto não vazio qualquer e $x \in X$ um ponto qualquer. Então $\{Y \subset$ $X: x \in Y\}$ é um ultrafiltro sobre $X$. De fato, seja $F=\{Y \subset X: x \in Y\}$ e suponha que exista $G \supseteqq F$, tal que $G$ seja filtro, então existe $A \in G$ tal que $x \notin A$, como $\{x\} \in F \subset G$ segue que $A \cap\{x\}=\emptyset \in G$ o que é um absurdo.

Proposição 1.56. Sejam $X$ um conjunto e $F$ um filtro sobre $X$. Então são equivalentes:

(1) F é ultrafiltro;

(2) Para todo $Y \subset X$, temos que ou $Y \in F$ ou $X \backslash Y \in F$.

Demonstração. Suponha que valha a condição (2) e que $F$ seja um filtro mas não seja um ultrafiltro, i.e., existe $G$ filtro tal que $F \varsubsetneqq G$. Seja $Y \in G \backslash F$, então $X \backslash Y \in F$ e daí $Y$ e $X \backslash Y \in F$ o que é um absurdo.

Reciprocamente, seja $F$ um filtro que não satisfaça (2), vamos mostrar que $F$ não é um ultrafiltro. Seja $Y \subset X$ tal que nem $Y$ e $X \backslash Y$ pertençam a $F$. Seja $G=F \cup\{Y\}$.

Afirmamos que $G$ tem p.i.f.. Se $A \in F$, então $A \cap Y \neq \emptyset$, caso contrário $A \subset X \backslash Y$ e $X \backslash Y \in F$. Assim, se $X_{1}, \ldots, X_{n} \in F$ implica que $X_{1} \cap \cdots \cap X_{n} \in F$ e portanto $Y \cap X_{1} \cap \cdots \cap X_{n} \neq \emptyset$. Portanto $G$ tem p.i.f.. Daí existe um filtro $F^{\prime} \supset G$. Como $Y \in F^{\prime} \backslash F$ então $F$ não é maximal, ou seja, $F$ não é ultrafiltro.

Dado um filtro, sempre existirá um ultrafiltro que o conterá.

Proposição 1.57. Seja $F$ um filtro em $X$. Então existe $G \supset F$ ultrafiltro sobre $X$.

Demonstração. Basta observar que dada uma cadeia de filtros $\left\{F_{\lambda}\right\}_{\lambda \in \Lambda}$ sobre $X$ com $F \subset F_{\lambda}$ a união $F^{\prime}=\bigcup_{\lambda \in \Lambda} F_{\lambda}$ é um filtro. Como $F^{\prime}$ é um limitante superior para a cadeia segue do Lema de Zorn que existe um filtro maximal $G \supset F$. 


\section{Capítulo}

\section{Noções Básicas de Topologia}

Este capítulo tem como função introduzir conceitos básicos de topologia geral que serão utilização ao longo da dissertação.

Definição 2.1. Dizemos que $(X, \tau)$ é um espaço topológico se $X$ é um conjunto $e$ $\tau \subset \wp(X)$ é tal que satisfaz:

(a) $X, \emptyset \in \tau$;

(b) se $A, B \in \tau$, então $A \cap B \in \tau$;

(c) se $\mathscr{A} \subset \tau$, então $\bigcup \mathscr{A} \in \tau$.

Cada elemento de $\tau$ é chamado de aberto.

Alguns problemas envolvendo a topologia podem ser difíceis de trabalhar ou simplesmente intratáveis. Algo que seria mais "tratável" é tentar obter os mesmos resultados usando menos abertos e isso refletir em toda topologia. Felizmente, existem tais objetos e são conhecidos como base de um espaço topológico.

Definição 2.2. Seja $(X, \tau)$ um espaço topológico. Dizemos que $\mathcal{B} \subset \tau$ é uma base para $(X, \tau)$ se para todo $x \in X$ e para todo $A \in \tau$ com $x \in A$ existir $B \in \mathcal{B}$ tal que $x \in B \subset A$.

Proposição 2.3. Uma família $\mathcal{B}$ de subconjuntos de $\tau$ é uma base para $(X, \tau)$, se, e somente se, para todo $A \in \tau \backslash\{\emptyset\}$ existir $\mathcal{B}^{\prime} \subset \mathcal{B}$ tal que $A=\bigcup \mathcal{B}^{\prime}$. 
Demonstração. Suponha que $\mathcal{B}$ seja uma base de $\tau$ e seja $A \in \tau \backslash\{\emptyset\}$. Para cada elemento $x \in A$, existe um conjunto $B_{x} \in \mathcal{B}$ tal que $x \in B_{x} \subset A$. Segue, então, que $A=\bigcup_{x \in A} B_{x}$.

Reciprocamente, seja $x \in X$ e $A \in \tau \operatorname{com} x \in A$. Como podemos escrever $A=$ $\cup \mathcal{B}^{\prime}$,tomamos $B \in \mathcal{B}^{\prime}$ tal que $x \in B$. Além disso, temos que $B \subset A$.

Definição 2.4. Dizemos que $(X, \tau)$ é $T_{1}$ se para $x, y \in X$ distintos existir um aberto $U$ tal que $x \in U$ e $y \notin U$.

Consideraremos todos os espaços em que trabalharemos $T_{1}$, caso contrário mencionaremos isso.

O conjunto $\mathbb{R}$ é um espaço topológico, com a topologia $\tau=\{A \subset \mathbb{R}:$ para todo $x \in A$ existe $\varepsilon>0$ tal que $] x-\varepsilon, x+\varepsilon[\subset A\}$. Esta é chamada de topologia usual em $\mathbb{R}$.

Definição 2.5. A reta $\mathbb{R}$ munido da topologia $\tau=\{A \subset \mathbb{R}:$ para todo $x \in A$, existe $r>0,\left[x, x+r[\subset A\}\right.$ é chamado de reta de Sorgenfrey e denotamos por $\mathbb{R}_{s}$.

Definição 2.6. Seja $(X, \leq)$ um conjunto totalmente ordenado. Chamamos de topologia da ordem sobre $(X, \leq)$ a topologia gerada pelos seguintes conjuntos (para todo $a, b \in X)$ :

(a) $] a, b[=\{x \in X: a<x<b\}$;

(b) $[a, b[=\{x \in X: a \leq x<b\}$, caso $a=\min X$;

(c) $] a, b]=\{x \in X: a<x \leq b\}$, caso $b=\max X$.

As topologias usuais sobre $\mathbb{R}, \mathbb{Q}, \mathbb{N}$ e $[0,1]$ são topologias induzidas pelas ordens usuais dos respectivos conjuntos.

Definição 2.7. Seja $(X, \tau)$ espaço topológico. Dizemos que X é conexo se, dados quaisquer abertos $A$ e $B$ de $X$ disjuntos tais que $A \cup B=X$ implicar que $A=\emptyset$ ou $B=\emptyset$.

Observe que a reta de Sorgenfrey não é conexa. Basta notar que $\{x \in \mathbb{R}: x<0\} \cup\{x \in$ $\mathbb{R}: 0 \leq x\}=\mathbb{R}$, com $\{x \in \mathbb{R}: x<0\},\{x \in \mathbb{R}: 0 \leq x\}$ abertos disjuntos e não vazios.

Proposição 2.8. Seja $(X, \leq)$ totalmente ordenado, conexo na topologia da ordem. Então qualquer $A \subset X$ não vazio e limitado possui supremo. 
Demonstração. Seja $A \subset X$ não vazio e limitado. Seja $B$ o conjunto dos limitantes superiores de $A$, i.e., para cada $b \in B$ temos que $a \leq b$ para todo $a \in A$.

Observe que :

- Se existir $a^{\prime} \in A$ tal que $a^{\prime}$ seja o máximo de $A$, então $a^{\prime}=\sup A$.

- Se existir $b^{\prime} \in B$ tal que $b^{\prime}$ seja o mínimo de $B$, então $b^{\prime}=\sup A$.

Agora, suponha que $A$ não tenha elemento máximo e que $B$ não tenha elemento mínimo. Note que $B \neq \emptyset$, pois caso contrário teríamos que $A=X$ e como $A$ é limitado (digamos por $b$ ) e $b \in X$ segue que $b \in A \operatorname{logo} A$ possui elemento máximo. Seja $\tilde{A}=\{x \in$ $X$ : existe $a \in A$ tal que $x \leq a\}$, note que $\tilde{A} \neq \emptyset$, pois $A \subset \tilde{A}, B \neq \emptyset, X=A \cup B$ e $A \cap B=\emptyset$. Afirmamos que $\tilde{A}$ e $B$ são abertos.

Para mostrar que $\tilde{A}$ é aberto. Seja $c \in \tilde{A}$, como $\tilde{A}$ não é limitado inferiormente existe $\xi^{\prime \prime} \in \tilde{A}$ tal que $\xi^{\prime \prime}<c$. Como $A$ não possui elemento máximo e $A \subset \tilde{A}$ então existe $\xi^{\prime} \in \tilde{A}$ tal que $c<\xi^{\prime}$. Assim $\left.c \in\right] \xi^{\prime \prime}, \xi^{\prime}[\subset \tilde{A}$. Portanto $\tilde{A}$ é aberto. De maneira análoga $B$ é aberto. Logo, exibimos uma cisão não trivial de $X$ o que é um absurdo tendo em vista que $X$ é conexo. Portanto existe $\sup A$.

Definição 2.9. Seja $\mathcal{F}$ uma família de funções da forma $f_{\alpha}: X \rightarrow Y_{\alpha}, \alpha \in A$, em que $X$ é um conjunto e cada $\left(Y_{\alpha}, \tau_{\alpha}\right)$ é um espaço topológico. Chamamos de topologia fraca induzida por $\mathcal{F}$ a topologia sobre $X$ gerada pelos conjuntos da forma $f_{\alpha}^{-1}[V]$, onde $\alpha \in A$ $e V \in \tau_{\alpha}$.

Definição 2.10. Seja $\left(\left(X_{\alpha}, \tau_{\alpha}\right)\right)_{\alpha \in A}$ um família de espaços topológicos. Defina o produto $\operatorname{dos}\left(\left(X_{\alpha}, \tau_{\alpha}\right)\right)_{\alpha \in A}$ como

$$
\prod_{\alpha \in A} X_{\alpha}=\left\{x: A \rightarrow \bigcup_{x \in A} X_{\alpha}: x(\alpha) \in X_{\alpha} \text { para todo } \alpha \in A\right\}
$$

com a topologia fraca induzida pelas funções $\left(\pi_{\alpha}\right)_{\alpha \in A}$ onde cada $\pi_{\alpha}: \prod_{\beta \in A} X_{\beta} \rightarrow X_{\alpha}$ é dada por $\pi_{\alpha}(x)=x(\alpha)$.

Esta topologia é chamada de topologia produto sobre $\prod_{\alpha \in A} X_{\alpha}$ (ou topologia de Tychonoff). 
Definição 2.11. Sejam $(X, \tau)$ um espaço topológico. Dizemos que $F \subset X$ é um conjunto fechado se $X \backslash F$ é aberto.

Em qualquer espaço topológico $(X, \tau), X$ e $\emptyset$ são fechados, pois seus complementares são abertos (em particular, $X$ e $\emptyset$ são abertos e fechados).

Em $\mathbb{R}$ o intervalo fechado $[0,1]$ é fechado na topologia usual.

Proposição 2.12. Seja $s \in 2^{<\omega}$ e seja $[s]=\left\{f \in 2^{\omega}: s \subset f\right\}$. Então $\left\{[s]: s \in 2^{<\omega}\right\}$ é uma base para a topologia produto de $2^{\omega}$

Demonstração. Seja $f \in 2^{\omega}$ e seja $V$ aberto da topologia produto tal que $f \in V$. Por $V$ ser um aberto da topologia produto, podemos escrevê-lo como $V=\prod_{n \in \omega} V_{n}$, onde temos que $V_{n}=\{0\}$ ou $V_{n}=\{1\}$ para $n \in\left\{n_{1}, \ldots, n_{k}\right\}$ e $V_{n}=2$ para $n \notin\left\{n_{1}, \ldots, n_{k}\right\}$. Sem perda de generalidade, suponha que $n_{1}<\cdots<n_{k}$ e tome $s: n_{k} \rightarrow 2\left(s \in 2^{<\omega}\right)$ tal que $s(n)=f(n)$. Portanto $f \in[s] \subset V$.

Definição 2.13. Seja $(X, \tau)$ um espaço topológico. Dizemos que $\mathcal{A}$ é uma cobertura (ou recobrimento) de $X$ se $\bigcup \mathcal{A}=X$. Chamamos de cobertura aberta se os elemento de $\mathcal{A}$ são abertos.

Definição 2.14. Dizemos que o espaço topológico $(X, \tau)$ é um espaço compacto se para toda cobertura aberta $\mathcal{A}$ existe uma subcobertura $\mathcal{A}^{\prime}$ (i.e., $\mathcal{A}^{\prime} \subset \mathcal{A}$ e $\cup \mathcal{A}^{\prime}=X$ ) finita.

Definição 2.15. Dizemos que o espaço topológico $(X, \tau)$ é um espaço localmente compacto se para todo ponto $x$ e todo aberto contento esse ponto $V$ existe um compacto $C$ tal que $x \in C \subset V$.

Definição 2.16. Seja $(X, \tau)$ um espaço topológico. Uma coleção $\mathcal{C} \subset X$ é dita ter a propriedade da intersecção finita (p.i.f) se para qualquer $C \subset \mathcal{C}$ finito, tivermos que $\bigcap C \neq \emptyset$.

Teorema 2.17. Seja X um espaço topológico. Então X é compacto, se, e somente se, para toda coleção $\mathcal{C}$ de fechados em $X$ com p.i.f. a intersecção $\bigcap \mathcal{C} \neq \emptyset$. 
Demonstração. Dada uma coleção $\mathcal{A}$ de subconjuntos de $X$, seja $\mathcal{C}=\{X \backslash A: A \in \mathcal{A}\}$. Observe que:

(1) $\mathcal{A}$ é uma coleção de abertos, se, e somente se, $\mathcal{C}$ é uma coleção de fechados;

(2) $\mathcal{A}$ cobre $X$, se, e somente se, $\bigcap \mathcal{C}=\emptyset$, pois $\bigcap_{A \in \mathcal{A}}(X \backslash A)=X \backslash\left(\bigcup_{A \in \mathcal{A}} A\right)=X \backslash X=\emptyset$;

(3) $\left\{A_{1}, \ldots, A_{n}\right\} \subset \mathcal{A}$ cobre $X$, se, e somente se, $\bigcap_{i=1}^{n}\left(X \backslash A_{i}\right)=\emptyset$.

O espaço $X$ é compacto se: "Dada qualquer coleção de abertos $\mathcal{A}$ de $X$, se $\mathcal{A}$ cobre $X$, então existe uma subcobertura finita de $\mathcal{A}$ que cobre $X$ ". Essa afirmação é equivalente a seguinte: "Dada qualquer coleção de abertos $\mathcal{A}$ de $X$, se não existe uma subcoleção finita de $\mathcal{A}$ que cobre $X$, então $\mathcal{A}$ não cobre $X$ ". Por sua vez, é equivalente a seguinte afirmação: "Dada qualquer coleção de fechados $\mathcal{C}$ de $X$, se $\mathcal{C}$ possui a p.i.f., então $\bigcap \mathcal{C} \neq \emptyset$. Com isso, segue o resultado do teorema.

Definição 2.18. Um espaço topológico $X$ é de Lindelöf se satisfaz a seguinte propriedade: toda cobertura aberta possui uma subcobertura enumerável.

Note que todo espaço compacto é de Lindelöf.

Veremos adiante que $\omega^{\omega}=\{f \mid f: \omega \rightarrow \omega\}$ também é de Lindelöf.

Definição 2.19. Seja $(X, \tau)$ um espaço topológico e $A \subset X$. Definimos o fecho de $A$ como sendo $\bar{A}=\bigcap\{F \subset X: F$ é fechado e $A \subset F\}$

Definição 2.20. Seja $(X, \tau)$ um espaço topológico e $A \subset X$. Dizemos que $x \in X$ é um ponto aderente à $A$ se para todo aberto $V$ tal que $x \in V$ tivermos $V \cap A \neq \emptyset$.

Lema 2.21. Seja $(X, \tau)$ um espaço topológico e $A \subset X$. Então $A \subset\{x \in X: x$ é ponto aderente $\grave{a} A\}$.

Demonstração. Naturalmente, se $x \in A$, qualquer que seja o aberto $U$ tal que $x \in U$, temos que $U \cap A \neq \emptyset$, pois $x \in U \cap A$.

Proposição 2.22. Seja $(X, \tau)$ um espaço topológico e $A \subset X$. Então o fecho de $A(\bar{A})$ é igual a $\{x \in X: x$ é ponto aderente à $A\}$. 
Demonstração. Chame de $D$ o conjunto dos pontos aderentes à $A$. Vamos mostrar que $\bar{A} \subset D$. Sejam $x \in \bar{A}$ e $V$ aberto tal que $x \in V$. Suponha que $V \cap A=\emptyset$, i.e., $A \subset X \backslash V$, que é fechado, logo, pela definição de $\bar{A}$, segue que $\bar{A} \subset X \backslash V$, contradição com o fato de que $x \in \bar{A}$ e $x \in V$.

Mostremos que $D \subset \bar{A}$. Seja $x \in D$. Suponha que $x \notin \bar{A}$, $\log 0 x \in X \backslash \bar{A}$ que é aberto, como $x \in D$, então $(X \backslash \bar{A}) \cap A \neq \emptyset$, o que é uma contradição, pois $A \subset \bar{A}$.

Definição 2.23. Seja $(X, \tau)$ um espaço topológico. Dizemos que $X$ é separável, se existe um subconjunto enumerável $D \subset X$ e denso em $X$, i.e., $\bar{D}=X$.

Lema 2.24. Seja $X$ um espaço topológico separável. Seja $A \subset X$ subespaço aberto, então A é separável.

Demonstração. Seja $D \subset X$ denso enumerável. Vamos mostrar que $A \cap D$ é denso em $A$. Seja $U$ um aberto em $X$ tal que $U \cap A \neq \emptyset$ (com isso $U \neq \emptyset$ ). Como $U \cap A$ é aberto e não vazio em $X$ (pois $A$ é aberto em $X$ ) segue que $(U \cap A) \cap(A \cap D)=(U \cap A) \cap D \neq \emptyset$.

Definição 2.25. Dizemos que $(X, \tau)$ é um espaço regular se para quaisquer $x \in X$ e $F \subset X$ fechado tais que $x \notin F$, existirem $A, B$ abertos tais que $x \in A, F \subset B$ e $A \cap B \neq \emptyset$.

Num espaço topológico regular, os abertos "separam" pontos de fechados.

Proposição 2.26. Sejam $(X, \tau)$ um espaço topológico. $O$ espaço $(X, \tau)$ é regular se, e somente se, para todo $x \in X$ e para todo aberto $V$ tal que $x \in V$ existir um aberto $A$ tal que $x \in A \subset \bar{A} \subset V$.

Demonstração. Suponha que $(X, \tau)$ seja regular. Sejam $x \in V$ e $V \in \tau$ tal que $x \in V$. Note que $X \backslash V$ é um fechado e $x \notin X \backslash V$. Então existem $A, B$ abertos disjuntos tais que $x \in A$ e $X \backslash V \subset B$.

Mostremos que $\bar{A} \subset V$ : De fato, $X \backslash B \subset V$ e $A \subset X \backslash B$ (pois $A \cap B=\emptyset$ ). Como $X \backslash B$ é fechado, temos $\bar{A} \subset X \backslash B \subset V$, e assim $x \in A \subset \bar{A} \subset V$.

Reciprocamente, mostremos que $(X, \tau)$ é regular. Sejam $x \in X$ e $F \subset X$ fechado tal que $x \notin F$. Então $X \backslash F$ é aberto e contém $x$. Logo, existe $A$ aberto tal que $x \in A \subset \bar{A} \subset X \backslash F$. Note que $x \in A, F \subset X \backslash \bar{A}$ e $A \cap(X \backslash \bar{A})=\emptyset$. 
Agora, vejamos o Teorema de Baire:

Teorema 2.27 (de Baire). A interseção de qualquer família enumerável de abertos densos em um espaço compacto e Hausdorff é densa.

Demonstração. Sejam $\left(A_{n}\right)_{n \in \omega}$ uma família de abertos densos e $V \neq \emptyset$ um aberto. Vamos mostrar que $V \cap\left(\bigcap_{n \in \omega} A_{n}\right) \neq \emptyset$. Para tanto, vamos construir uma sequência $\left(V_{n}\right)_{n \in \omega}$ de abertos não vazios tais que:

(1) $\overline{V_{0}} \subset V$;

(2) $\overline{V_{n}} \subset A_{n}$, para todo $n \in \omega$;

(3) $\overline{V_{n+1}} \subset V_{n}$, para todo $n \in \omega$.

Como $A_{0} \cap V \neq \emptyset$ é aberto, existe $x_{0} \in A_{0} \cap V$. Por $X$ ser regular (pois é compacto e Hausdorff), existe $V_{0}$ tal que $x_{0} \in V_{0} \subset \overline{V_{0}} \subset A_{0} \cap V$. Note que foram satisfeitos os ítens (1), (2) e (3) acima (o último por vacuidade).

Suponha definidos $V_{n}$, para $n \in \omega$, satisfazendo as condições acima. Definamos $V_{n+1}$. Como $V_{n} \cap A_{n+1} \neq \emptyset$ é aberto, existe $x_{n+1} \in V_{n} \cap A_{n+1}$. Novamente, pela regularidade de $X$, existe $V_{n+1}$ aberto tal que $x_{n+1} \in V_{n+1} \subset \overline{V_{n+1}} \subset V_{n} \cap A_{n+1}$, que claramente satisfaz (2) e (3). Isto encerra a indução.

Note que a família $\left(\overline{V_{n}}\right)_{n \in \omega}$ é uma família de fechados com p.i.f. (devido a condição (3)), em um espaço compacto. Logo, existe $x \in \bigcap_{n \in \omega} \overline{V_{n}}$. Por (1) e (2), $x \in V \cap\left(\bigcap_{n \in \omega} A_{n}\right)$.

Corolário 2.28. A interseção de qualquer familia enumerável de abertos densos em um espaço localmente compacto e Hausdorff é densa.

Demonstração. Basta repetir o que foi feito no teorema anterior para uma vizinhança compacta.

Definição 2.29. Dizemos que $(X, \tau)$ é um espaço de Baire se para toda familia $\left(A_{n}\right)_{n \in \omega}$ de abertos densos em $X$ tivermos que $\bigcap_{n \in \omega} A_{n}$ é denso em $X$. 


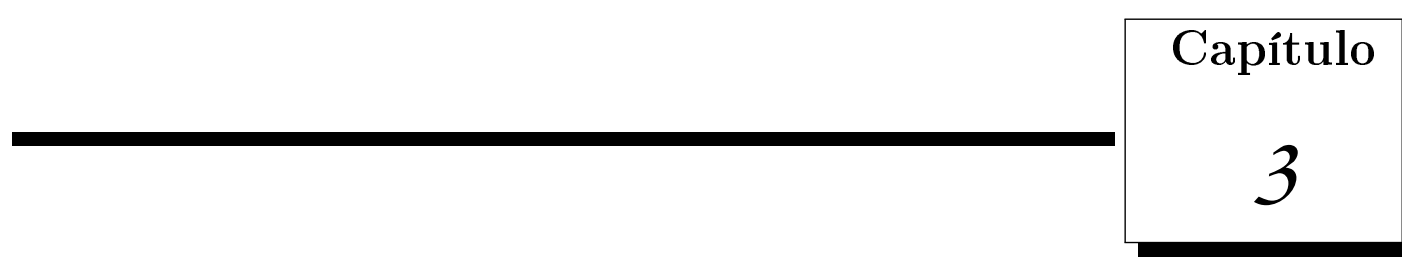

\section{Dois Axiomas Extras à ZFC}

Nesse capítulo introduziremos dois novos axiomas, o axioma de Martin $(M A)$ e o Princípio diamante $(\diamond)$. Esses axiomas além de serem importantes para os mais variados campos da matemática, como teoria de conjuntos e topologia geral, serão muito úteis no capítulo 5A Hipótese de Suslinchapter.5 para provar a independência da Hipótese de Suslin $(S H)^{1}$.

\subsection{O Axioma de Martin}

Em $Z F C$ temos o seguinte resultado para ordens:

Proposição 3.1. Se $(P, \preceq)$ é um conjunto ordenado não vazio e para cada $n \in \omega, D_{n} \subset P$ é denso em $P$, então existe $F \subset P$ filtro tal que $F \cap D_{n} \neq \emptyset$ para todo $n \in \omega$.

Demonstração. Seja $d_{1} \in D_{1} \subset P$. Como $D_{2}$ é denso em $P$ então existe $d_{2} \in D_{2}$ tal que $d_{2} \preceq d_{1}$ e daí, como $D_{3}$ é denso em $P$, então existe $d_{3} \in D_{3}$ com $d_{3} \preceq d_{2}$. Continuando esse processo teremos que existe $d_{n} \in D_{n}$ tal que $d_{n} \preceq d_{n-1}$, com $d_{n-1} \in D_{n-1}$.

Seja $G$ o conjunto formado por esses termos, ou seja, $G=\left\{d_{1}, d_{2}, d_{3}, \ldots, d_{n}, \ldots\right\}$. Daí por $G$ ser uma cadeia, isto é, todos os elementos são comparáveis, então $G$ possui p.i.f..

\footnotetext{
${ }^{1}$ I.e. se assumirmos $S H$ ou $\neg S H$ não temos uma contradição com $Z F C$.
} 
Assim, pela Observação 1.54Filtros e Ultrafiltrosteorema.1.54 existe um filtro $F$ tal que $G \subset F$ e ainda $F \cap D_{n} \neq \emptyset$ para todo $n \in \omega$.

O que acontece se quisermos que o mesmo aconteça trocando $\omega$ por $\omega_{1}$, por exemplo?

Definiremos uma condição necessária para o Axioma de Martin.

Definição 3.2. Seja $(P, \leq)$ um conjunto ordenado. Dizemos que é c.c.c. se toda anticadeia for enumerável.

A c.c.c. possui propriedades interessantes. Veremos algumas propriedades e aplicações no Capítulo 7Produto de espaços c.c.cchapter.7.

Definição $3.3(M A)$. Chamamos de axioma de Martin a seguinte afirmação:

"Seja $(P, \leq)$ é um conjunto ordenado não vazio c.c.c.. Se $\kappa<2^{\omega}$ e, para cada $\varepsilon<\kappa$, $D_{\varepsilon}$ for denso em $P$, então existe $F \subset P$ filtro tal que, para todo $\varepsilon<\kappa, F \cap D_{\varepsilon} \neq \emptyset$."

O axioma de Martin foi concebido por Donald A. Martin e Robert M. Solovay em 1970. $\mathrm{Na}$ tentativa de provar a consistência ${ }^{2}$ dos três seguintes problemas quando assumimos $\neg C H$ :

(1) Se $\kappa<2^{\omega}$ então toda família quase disjunta (q.d.) $\mathscr{A} \subset \wp(\omega)^{3}$ de cardinalidade $\kappa$ não é maximal?

(2) Se $\kappa<2^{\omega}$, então a união de $\kappa$ subconjuntos de $\mathbb{R}$ de medida de Lebesgue nula tem medida de Lebesgue nula?

(3) Se $\kappa<2^{\omega}$, então a união de $\kappa$ subconjuntos de $\mathbb{R}$ de $1^{\text {a }}$ categoria é de $1^{\text {a }}$ categoria ${ }^{4}$ ?

Por meio da técnica de "Forcing Iterado" 5 desenvolvida por Solovay e Tennenbaum notou-se que que a resposta dessas três questões seriam positivas. O matemático Donald A. Martin notou que a técnica usada para gerar essas respostas positivas poderia ser reduzida em um axioma. Tal axioma recebeu o seu nome Axioma de Martin $(M A)$.

\footnotetext{
${ }^{2}$ I.e. se não gera contradições em $Z F C+\neg C H$.

${ }^{3}$ Seja $\kappa$ um cardinal regular (veja a Definição 1.23Ordinais e Cardinaisteorema.1.23). Se $x, y \subset \kappa$, então $x$ e $y$ são quase disjuntos (q.d.) se, e somente se, $|x \cap y|<\kappa$. Uma família $\mathscr{A} \subset \wp(\kappa)$ é q.d. se para todo $x \in \mathscr{A}$ implicar que $|x|=\kappa$ e se para quaisquer dois elementos distintos de $\mathscr{A}$ eles são q.d..

${ }^{4}$ Veja a definição na página 110Espaços de Blumbergteorema.9.2.

${ }^{5}$ Para entender como funciona a técnica veja [11].
} 
Como $M A$ pode ser compreendido sem o conhecimento do método de Forcing ele se tornou um axioma importante na prova de consistência de questões que envolvam cardinais $\kappa$ onde $\omega<\kappa<2^{\omega}$. Atráves do método de forcing iterado provou-se que $M A$ é independente dos axiomas usuais de $Z F C$.

Agora, daremos uma aplicação de $M A$ em um teorema que é uma generalização do Teorema de Baire.

Definição 3.4. Dizemos que um espaço topológico $(X, \tau)$ satisfaz c.c.c. se $\left(\tau^{*}, \subset\right)^{6}$ satisfaz c.c.c.

Teorema $3.5(M A)$. Seja X um espaço topológico compacto Hausdorff c.c.c.. Se $\kappa<2^{\omega}$ e, para cada $\varepsilon<\kappa, D_{\varepsilon}$ é aberto denso em $X$, então $\bigcap_{\varepsilon<\kappa} D_{\varepsilon}$ é denso em $X$.

Faremos a demostração deste Teorema com o auxílio do seguinte lema:

Lema 3.6. Seja $(X, \tau)$ um espaço topológico regular e $\left(\tau^{*}, \subset\right)$ o conjunto formado pelos abertos não vazios de $X$ ordenado pela inclusão. Se $A$ é aberto denso em X, então $A^{\prime}=\left\{V \in \tau^{*}: \bar{V} \subset A\right\}$ é denso em $\left(\tau^{*}, \subset\right)$.

Demonstração. Vamos mostrar que para todo $U \in \tau^{*}$ existe $V \in A^{\prime}$ tal que $V \subset U$.

Note que, $X$ ser regular é equivalente à, dado um ponto $x \in X$ e uma vizinhança aberta $U$ de $x$, existir uma vizinhança aberta $V$ tal que $x \in V \subset \bar{V} \subset U$.

Como $\bar{A}=X$, então $U \cap A \neq \emptyset$ para todo $U \in \tau^{*}$. Por $U \cap A$ ser aberto não vazio segue que existe $V \in \tau^{*}$ tal que $V \subset \bar{V} \subset U \cap A \subset U$.

Demostração do Teorema 3.50 Axioma de Martinteorema.3.5. Sejam $\left(D_{\xi}\right)_{\xi<\kappa}$ uma família de abertos densos em $X$. Então cada $D_{\xi}^{\prime}=\left\{V \in \tau^{*}: \bar{V} \subset D_{\xi}\right\}$ é denso em $\left(\tau^{*}, \subset\right)$. Pelo axioma de Martin, existe um filtro $F$ tal que $F \cap D_{\xi}^{\prime} \neq \emptyset$ para cada $\xi<\kappa$. Como $F$ é filtro, então $F$ tem p.i.f.. Assim, considere $G=\{\bar{V}: V \in F\}$ e note que, por sua vez, $G$ também tem p.i.f.. Como $X$ é compacto, então $\bigcap_{V \in G} \bar{V} \neq \emptyset$. Seja $x \in \bigcap_{V \in G} \bar{V}$. Observe que $\bigcap_{V \in G} \bar{V} \subset \bigcap_{\xi<\kappa} V_{\xi}$ onde, para cada $\xi<\kappa, V_{\xi} \in \tau^{*}$ é tal que $V_{\xi} \in F \cap D_{\xi}^{\prime}$. Logo $x \in \bigcap_{\xi<\kappa} D_{\xi}$, e portanto, $\bigcap_{\xi<\kappa} D_{\xi} \neq \emptyset$.

$$
{ }^{6} \tau^{*}=\tau \backslash\{\emptyset\} .
$$


Feitas todas essas considerações, agora provemos de fato o teorema. Seja $U \in \tau^{*}$, então tome $x \in U$. Daí, como $X$ é compacto segue que existe uma vizinhança compacta $W$ tal que $W \subset U$. Observe que cada $D_{\xi} \cap W$ para $\xi<\kappa$ é denso em $W$.

Analogamente, temos que $\bigcap_{\xi<\kappa}\left(W \cap D_{\xi}\right) \neq \emptyset$, como $\bigcap_{\xi<\kappa}\left(W \cap D_{\xi}\right)=W \cap\left(\bigcap_{\xi<\kappa} D_{\xi}\right) \subset$ $U \cap\left(\bigcap_{\xi<\kappa} D_{\xi}\right)$, segue que $U \cap\left(\bigcap_{\xi<\kappa} D_{\xi}\right) \neq \emptyset$. Portanto $\bigcap_{\xi<\kappa} D_{\xi}$ é denso em $X$.

Corolário 3.7 (MA). Seja X um espaço topológico localmente compacto, Hausdorff c.c.c.. Se $\kappa<2^{\omega}$ e, para cada $\varepsilon<\kappa, D_{\varepsilon}$ é aberto denso em $X$, entãa $\bigcap_{\varepsilon<\kappa} D_{\varepsilon}$ é denso em $X$.

Observe que o Axioma de Martin somente será interessante junto com a negação da Hipótese do Contínuo $(M A+\neg C H)$, pois, caso contrário, o único $\kappa<2^{\omega}$ é o próprio $\omega$ e, portanto já temos o resultado em 3.10 Axioma de Martinteorema.3.1. Além disso, não podemos mostrar o análogo para $\kappa=2^{\omega}$, pois considere $\mathbb{R}$ com a topologia usual e a seguinte família de abertos densos $\mathscr{D}=\{\mathbb{R} \backslash\{x\}: x \in \mathbb{R}\}$, porém $\bigcap \mathscr{D}=\emptyset$ contradizendo o Corolário 3.7O Axioma de Martinteorema.3.7.

\subsection{Clubs e Conjuntos Estacionários}

Nessa seção estudaremos os pré-requisitos necessários para entender o Princípio $\diamond$ e ao final daremos uma aplicação desses conceitos, o Lema do "Pressing Down".

Adotaremos nos ordinais a topologia da ordem.

Proposição 3.8. Para qualquer ordinal limite $\mu$, um conjunto $C \subset \mu$ é fechado se, $e$ somente se, para todo limite $\delta<\mu$, se $C \cap \delta$ é ilimitado em $\delta$, então $\delta \in C$.

Demonstração. Seja $C \subset \mu$ fechado na topologia da ordem e seja $\delta<\mu$ tal que $C \cap \delta$ é ilimitado em $\delta$, i.e. para todo $\gamma<\delta$ existe $\eta \in C \cap \delta$ tal que $\gamma<\eta$.

Mostremos que $\delta \in \bar{C}$, pois $\bar{C}=C$ já que $C$ é fechado. Para qualquer vizinhança aberta $V$ de $\delta$ podemos tomar $] \xi, \delta]$ aberto tal que $\delta \in] \xi, \delta] \subset V$. Por $C \cap \delta$ ser ilimitado em $\delta$ (para todo $\gamma$ existe $\eta \in C \cap \delta$ tal que $\gamma<\eta$ ), em particular $\xi<\delta$, existe $\zeta \in C \cap \delta$ tal que $\xi<\zeta$. Assim, $] \xi, \delta] \cap C \neq \emptyset$ e portanto $\delta \in \bar{C}=C$. 
Reciprocamente, seja $C \subset \mu$. Suponha que exista $\gamma \in \bar{C} \backslash C$. Então $\gamma$ não poderia ser sucessor, pois se $\gamma=\alpha+1$ bastaria tomar o aberto $] \alpha, \gamma]$ e assim $] \alpha, \gamma] \cap C=\emptyset$ e então $\gamma \notin \bar{C}$. Logo $\gamma$ é limite e então $C \cap \gamma$ é ilimitado em $\gamma$. De fato, suponha que não, então existe $\kappa<\gamma$ tal que $\alpha<\kappa$ para todo $\alpha \in C \cap \gamma$. Assim $] \kappa, \gamma] \cap C=\emptyset$ e portanto $\gamma \notin \bar{C}$. Logo, pela definição de $C$, temos $\gamma \in C$, o que também é um absurdo. Portanto $C$ é fechado na topologia da ordem.

Definição 3.9. Seja $\alpha$ é um ordinal limite. Dado $C \subset \alpha$ dizemos que $C$ é um club (closed unbonded) se, e somente se, $C$ é fechado e ilimitado.

Alguns exemplos de conjuntos clubs:

Seja $\mu$ um ordinal.

(1) Seja $C=\{\gamma<\mu: \gamma$ é ordinal limite $\}$. Seja $\gamma \in \bar{C}$. Suponha que $\gamma$ seja sucessor, digamos que $\gamma=\alpha+1$, então $] \alpha, \gamma] \cap C=\emptyset, \log o \gamma \notin \bar{C}$ o que é um absurdo. Portanto $C$ é fechado.

(2) $D=\{\gamma<\mu: \gamma$ é limite de ordinais limites $\}$. $D$ é fechado. De fato, seja $\delta<\mu$ tal que $C \cap \delta$ seja ilimitado em $\delta$. Observe que o limite de ordinais limite é um ordinal limite. Como para cada $\kappa<\delta$ existe $\xi \in C \cap \delta$ tal que $\kappa<\xi<\delta$ segue que $\delta$ é um limite de ordinais limite e portanto $\delta \in C$. Assim, pela Proposição 3.8Clubs e Conjuntos Estacionáriosteorema.3.8 temos que $C$ é fechado.

Se $\mu$ é um cardinal maior que $\omega$ então (1) e (2) são clubs. Mostremos que $C$ é ilimitado. De fato, seja $\xi<\mu$ e suponha que $\xi=\alpha+1$, i.e., $\xi$ é um ordinal sucessor. Seja $\xi^{\prime}=\sup _{n \in \omega} \alpha+n$. Agora, como $\mu$ é um cardinal e é maior que $\omega$ teremos que pelo menos $\omega_{1} \leq \mu$. Como $\xi^{\prime}<\omega_{1}$ segue que $\xi^{\prime}<\mu$ e portanto $C$ é ilimitado. Para mostrar que o conjunto $D$ é ilimitado é análogo à $C$. Observe que se $\mu<\omega$ não garantiríamos que tais conjuntos fossem ilimitados.

Se $\mu$ é um cardinal limite, então $E=\{\gamma<\mu: \gamma$ é um cardinal $\}$ é um club em $\mu$. Note que $0,1,2,3, \ldots, \omega, \omega_{1}, \omega_{2}, \ldots, \omega_{n}, \ldots$ são elementos de $E$ para, digamos, $\mu=\omega_{\omega}$. Para ver que $E$ é fechado, seja $\gamma \in \bar{E} \backslash E$, então para todo $\xi<\gamma$ temos que $] \xi, \gamma] \cap E \neq \emptyset$. Observe que: 
- se $\gamma$ for sucessor $(\gamma=\alpha+1)$ então $] \alpha, \gamma] \cap E \neq \emptyset$ e então $\gamma \in E$,

- se $\gamma$ for limite a prova é a mesma feita para o conjunto $C$.

Para mostrar que $E$ é ilimitado seja $\alpha<\mu$, como a princípio $\alpha$ é somente um ordinal (não necessariamente um cardinal) temos que $|\alpha|=\kappa$, onde $\kappa \leq \alpha$. Tome $\kappa^{+}$e observe que $\alpha<\kappa^{+}$, pois $\kappa<\kappa^{+}$e $\kappa^{+}$não tem bijeção com $\kappa$. Para finalizar, temos que $\kappa^{+}<\mu$, caso contrário, se $\mu<\kappa^{+}$então $\mu \leq \kappa$ o que é um absurdo e também não podemos ter que $\mu=\kappa^{+}$, pois $\mu$ é ordinal limite (lembrando que $\kappa^{+}=\min \{\beta: \beta$ é cardinal e $\kappa<\beta\}$ ).

Definição 3.10. Seja $\mu$ um ordinal tal que $\operatorname{cf}(\mu)>\omega$. O filtro club em $\mu, C l u b(\mu) e ́$ o conjunto

$$
\{X \subset \mu \text { : existe } C \subset X \text { tal que } C \text { é club em } \mu\}
$$

Note que os elementos de $C l u b(\mu)$ não necessariamente são $c l u b s$. De fato, seja $C=$ $\left\{\gamma<\omega_{1}: \gamma\right.$ é limite e $\left.\gamma>\omega\right\}$. Seja $X=C \cup \omega$, então $X \in C l u b\left(\omega_{1}\right)$, pois $C$ é um $c l u b$ em $\omega_{1}$. De fato,

- $C$ é fechado em $\omega_{1}$. Seja $\alpha \in \bar{C}$, para cada $\left.\left.\xi<\alpha\right] \xi, \alpha\right] \cap C \neq \emptyset$. Tome $\left.\left.\gamma_{\xi} \in\right] \xi, \alpha\right] \cap C$ para cada $\xi$, então $\alpha$ é limite e $\alpha>\omega$, pois $\gamma_{\xi}<\alpha$ e $\omega<\gamma_{\xi}$. Portanto $\alpha \in C$. Portanto $C$ é fechado.

- $C$ é ilimitado em $\omega_{1}$. Seja $\alpha<\omega_{1}$ tal que $\omega<\alpha<\omega_{1}$. Seja $\kappa=\min \{\gamma: \gamma$ é limite e $\left.\alpha<\gamma<\omega_{1}\right\}$. Tal $\kappa$ de fato existe, pois caso contrário, existiriam somente ordinais sucessores de $\alpha$ até $\omega_{1}$.

Porém $X$ não é $c l u b$ em $\omega_{1}$. Pois o ponto $\omega \in \bar{X} \backslash X$.

O $C l u b(\mu)$ tem pelo menos uma chance de ser filtro, uma vez que as condições $(a)$ e (c) da Definição 1.51Filtros e Ultrafiltrosteorema.1.51 são facilmente verificadas. De fato:

- $\emptyset \notin C l u b(\mu)$, pois $\emptyset$ não é ilimitado.

- Sejam $A \in C l u b(\mu)$ e $B \subset \mu$ tal que $A \subset B$. Como $A \in C l u b(\mu)$ então existe $C \subset A$ tal que $C$ é $c l u b$ em $\mu$. Como $C \subset A \subset B$ então $B \in C l u b(\mu)$. 
Agora mostraremos que $C l u b(\mu)$ satisfaz a condição $(b)$ da Definição 1.51Filtros e Ultrafiltrosteorema.1.51 e ainda mais:

Definição 3.11. Seja $\kappa$ um cardinal. Um filtro $F$ é dito ser $\kappa$-completo se, e somente se para todo $\mathscr{A} \subset F$ com $|\mathscr{A}|<\kappa$ tivermos $\bigcap \mathscr{A} \in F$.

Lema 3.12. Seja $\mu$ um ordinal limite. Se $\operatorname{cf}(\mu)>\omega$, então:

(1) a intersecção de qualquer família de menor que $c f(\mu)$ de subconjuntos clubs de $\mu$ é um club;

(2) $\operatorname{Club}(\mu)$ é um $\mathrm{cf}(\mu)$-filtro completo.

Demonstração. Para o ítem $(a)$ seja $C_{\alpha}$ um $c l u b$ em $\mu$ para $\alpha<\lambda$, onde $\lambda<c f(\mu)$ e seja $D=\bigcap_{\alpha<\lambda} C_{\alpha}$

(i) $D$ é fechado, pois é a intersecção de fechados.

(ii) Para mostrar que $D$ é ilimitado, em primeiro lugar, para cada $\xi \in C_{\alpha}$ seja $f_{\alpha}: \mu \rightarrow \mu$ onde $f_{\alpha}(\xi)$ é o menor elemento de $C_{\alpha}$ maior que $\xi$. Seja $g(\xi)=\sup \left\{f_{\alpha}(\xi): \alpha<\lambda\right\}$, então $\xi<g(\xi)<\mu$, pois $\lambda<c f(\mu)$ e em virtude disso $g$ não atinge $\mu$. Seja $g^{0}(\xi)=\xi, g^{n+1}(\xi)=g\left(g^{n}(\xi)\right)$ e $g^{\omega}(\xi)=\sup \left\{g^{n}(\xi): n<\omega\right\}$, então $\xi<g^{\omega}(\xi)<\mu$. Para cada $\alpha<\lambda, C_{\alpha}$ é ilimitado em $g^{\omega}(\xi)$. De fato, seja $\gamma<\mu \operatorname{com} \gamma<g^{\omega}(\xi)$, então existe algum $n$ tal que $\gamma<g^{n}(\xi):=\xi^{\prime}<g^{\omega}(\xi)$. Se $\xi^{\prime} \in C_{\alpha}$ acabou. Senão, temos que $f_{\alpha}\left(\xi^{\prime}\right) \in C_{\alpha}$ e $\gamma<g^{n}(\xi) \leq f_{\alpha}\left(\xi^{\prime}\right) \leq g\left(\xi^{\prime}\right) \leq g^{\omega}(\xi)<\mu$. Então $g^{\omega}(\xi) \in C_{\alpha}$, logo $g^{\omega}(\xi) \in D$. Portanto, para cada $\xi<\mu$ temos que $\xi<g^{\omega}(\xi)<\mu$.

Agora, para mostrar o ítem (b), seja $X_{\alpha} \in C l u b(\mu)$ para cada $\alpha<\lambda$, onde $\lambda<c f(\mu)$. Tome club $C_{\alpha} \subset X_{\alpha}$, então por $\left.a\right) \bigcap_{\alpha} C_{\alpha}$ é club e $\bigcap_{\alpha} C_{\alpha} \subset \bigcap_{\alpha} X_{\alpha}$ e portanto $\bigcap_{\alpha} X_{\alpha} \in$ $C l u b(\mu)$.

Veremos o que é um conjunto estacionário e alguns lemas para nos preparar para a demonstração do Lema do "Pressing Down".

Definição 3.13. Seja $\mu$ um ordinal. Se $c f(\mu)>\omega, X \subset \mu$ é estacionário se, e somente se, $X \cap C \neq \emptyset$ para todo $C \subset \mu$ club. 
Observação 3.14. Se $X \subset \mu$ é estacionário então $X$ é ilimitado. Caso contrário existiria $\alpha<\mu$ onde $\kappa<\alpha$ para todo $\kappa \in X$ e sendo assim defina $C=\{\gamma<\mu: \gamma$ é limite $e$ $\gamma>\alpha\}$. Então $C$ é um club e $X \cap C=\emptyset$.

Vejamos um exemplo de conjunto estacionário. Se $\operatorname{cf}(\mu)>\lambda$, onde $\lambda$ é regular, então $\{\gamma<\mu: c f(\gamma)=\lambda\}$ é estacionário em $\mu$.

De fato, seja $C$ um $c l u b$ em $\mu$ e seja $\gamma \in C$ o seu $\lambda$-ésimo elemento (observe que tal elemento existe, pois $C$ é ilimitado). Vamos mostrar que $c f(\gamma)=\lambda$. A princípio, sabemos que $c f(\gamma) \leq \lambda^{7}$. Suponha, por absurdo, que $\beta=c f(\gamma)<\lambda$. Seja $f: \beta \rightarrow \gamma$ crescente e ilimitada e seja $g: \beta \rightarrow \lambda$ dada por $g(b)=\min \left\{\xi: x_{\xi}>f(b)\right\}$, onde $\left\{x_{\xi} \in C: \xi<\lambda\right\}$.

- $g$ está bem definida. Seja $b \in \beta$, $\log 0 f(b)<\gamma$ então existe $x_{\xi} \in C \operatorname{com} \xi<\lambda$ tal que $x_{\xi}>f(b)$.

- $g$ é ilimitada. Seja $\alpha<\lambda$ e tome $x_{\alpha}<\gamma$. Como $f$ é ilimitada existe $\eta<\beta$ tal que $x_{\alpha}<f(\eta)<\gamma$. Mas $g(\eta)=\min \left\{\xi: x_{\xi}>f(\eta)\right\}$ e daí seja $\eta^{\prime}<\lambda$. tal que $x_{\alpha}<f(\eta)<x_{\eta^{\prime}}$, onde $g(\eta)=\eta^{\prime}$. Então $\alpha<\eta^{\prime}=g(\eta)<\lambda$.

$\operatorname{Logo} \lambda=c f(\lambda) \leq \beta$ o que é um absurdo. Portanto $c f(\gamma)=\lambda$

Definição 3.15. Uma função n-ária em $A$ é uma $f: A^{n} \rightarrow A$ se $n>0$, ou um elemento de $A$ se $n=0$. Uma função finita é uma função n-ária para algum $n$. Se $B \subset A, B$ é fechado sob $f$ se, e somente se, $f\left(B^{n}\right) \subset B$. Se $\mathscr{J}$ é um conjunto de funções finitas e $B \subset A$, o fecho de $B$ sob $\mathscr{J}$ é o "menor" $C \subset A$ tal que $B \subset C$ e $C$ é fechado sob todas as funções em $\mathscr{J}$. Note que existe pelo menos um $C$, a saber

$$
C=\bigcap\{D: B \subset D \subset A \text { e D é fechado em } \mathscr{J}\} .
$$

Lema 3.16. Seja $\kappa>\omega$ regular e seja $\mathscr{A}$ um conjunto de funções finitas em $\kappa$ tal que $|\mathscr{A}|<\kappa ;$ então $C=\{\gamma<\kappa: \gamma$ é fechado sob $\mathscr{A}\}$ é club em $\kappa$.

Demonstração.

\footnotetext{
${ }^{7}$ Por $\gamma$ ser o $\lambda$-ésimo elemento de $C$.
} 
- $C$ é fechado. Seja $\gamma \in \bar{C}$, então para todo $\xi<\gamma] \xi, \gamma] \cap C \neq \emptyset$. Note que para cada $\eta \in] \xi, \gamma] \cap C$ temos que $f(s)<\eta$ para todo $s \in \operatorname{dom} f \mid \eta$ e para todo $f \in \mathscr{A}$. Seja $f \in \mathscr{A}$ e seja $D=\operatorname{dom} f 1 \gamma$ que por sua vez é finito, então considere $D=\left\{a_{j}: j=1, \ldots, n\right\}$ onde $a_{i}<a_{j}$ se $i<j$. Observe que:

1. Se $a_{n}<\gamma$ tome $\left.\left.\eta \in\right] a_{n}, \gamma\right] \cap C$ e então $f(s)<\eta$ para todo $s \in \operatorname{dom} f|\eta=\operatorname{dom} f| \gamma$ e portanto $\gamma$ é fechado sob $f$.

2. Se $a_{n}=\gamma$ suponha por absurdo que $f\left(a_{n}\right)>\gamma$ e considere $\left[\gamma, f\left(a_{n}\right)[\cap C\right.$ que é diferente de vazio. Então para todo $\eta \in\left[\gamma, f\left(a_{n}\right)\left[\cap C\right.\right.$ temos que $\eta<f\left(a_{n}\right)$ o que é um absurdo. $\operatorname{Logo} f\left(a_{n}\right)<\gamma$. Portanto $\gamma \in C$.

- $C$ é ilimitado. Seja $\xi<\kappa$ e tome $G(\xi)$ o fecho de $\xi$ sob $\mathscr{A}$, ou seja $f(G(\xi) \cap \operatorname{dom} f) \subset$ $G(\xi)$ para todo $f \in \mathscr{A}$, então $\xi \subset G(\xi) \subset \kappa$, e $|G(\xi)|<\kappa$ por (I 10. 23) de [11]. Como $\kappa$ é regular, podemos tomar $g(\xi)$ tal que $\xi<g(\xi)<\kappa$ e $G(\xi) \subset g(\xi)$. Como na prova do Lema 3.12Clubs e Conjuntos Estacionáriosteorema.3.12, seja $g^{n}$ o $n$ ésimo iterado de $g$ e $g^{\omega}(\xi)=\sup _{n<\omega} g^{n}(\xi)$. Então $g^{\omega}(\xi)$ é um elemento de $C$ maior que $\xi$.

Lema 3.17. Seja $\kappa>\omega$ regular e seja $C_{\alpha}$ club em $\kappa$ para todo $\alpha<\kappa$, então

$$
D=\left\{\gamma \text { :para todo } \alpha<\gamma \text { implica que } \gamma \in C_{\alpha}\right\}
$$

é um club.

Demonstração.

- $D$ é fechado. Seja $\gamma \in \bar{D}$. Seja $\alpha<\gamma$ e considere $] \alpha, \gamma]$, então $] \alpha, \gamma] \cap D \neq \emptyset$, $\log o$ existe $\eta \in D$ tal que $\alpha<\eta<\gamma$ e assim $\eta \in C_{\alpha}$, então $\left.] \alpha, \gamma\right] \cap C_{\alpha} \neq \emptyset \Rightarrow \gamma \in \overline{C_{\alpha}}=C_{\alpha}$. Portanto $\gamma \in D$.

- $D$ é ilimitado. Seja $g(\xi) \in \bigcap_{\alpha<\xi} C_{\alpha}$ maior que $\xi\left(\bigcap_{\alpha<\xi} C_{\alpha}\right.$ é ilimitado pelo Lema 3.12Clubs e Conjuntos Estacionáriosteorema.3.12). Então o elemento $g^{\omega}(\xi)$ (definido pelo Lema 3.16Clubs e Conjuntos Estacionáriosteorema.3.16) pertence a $D$ e $\xi<$ $g^{\omega}(\xi)$. 
Em teoria da medida temos (por exemplo em $[0,1]$ ) três tipos de conjuntos: os pequenos que consistem de conjuntos de medida nula, os médios que são os de medida positiva e os grandes que são os conjuntos que possuem medida unitária (total). Temos o seguinte resultado: Um conjuntos grande intercepta todos os conjuntos médios. Podemos usar essa analogia no contexto dessa seção, os conjuntos médios corresponderão aos clubs e os grandes serão os conjuntos estacionários.

Definição 3.18. Seja $\kappa$ um ordinal e seja $f: S \rightarrow \kappa$. Dizemos que $f$ é regressiva se para todo $\gamma \in S$ implica que $f(\gamma)<\gamma$.

Lema 3.19 (Lema do "Pressing Down"). Seja $\kappa>\omega$ regular, $S$ um subconjunto estacionário de $\kappa$ e $f: S \rightarrow \kappa$ regressiva. Então existe $\alpha<\kappa$ tal que $f^{-1}\{\alpha\}$ é estacionário.

Demonstração. Suponha que não exista $\alpha<\kappa$ tal que $f^{-1}\{\alpha\}$ seja estacionário. Então para cada $\alpha$ tome $c l u b C_{\alpha}$ tal que $C_{\alpha} \cap f^{-1}\{\alpha\}=\emptyset$. Seja $D=\{\gamma$ : para todo $\alpha<\gamma$ implica que $\gamma \in C_{\alpha}$ \} e note que $D$ é um club pelo Lema anterior, mas $D \cap S=\emptyset$, pois se existir $\gamma \in D \cap S, f(\gamma) \neq \alpha$ para cada $\alpha<\gamma^{8}$ contradizendo $f(\gamma)<\gamma$. Logo, $S$ não seria estacionário. Portanto existe $\alpha<\kappa$ tal que $f^{-1}\{\alpha\}$ é estacionário.

Podemos ler tal lema como: se uma função sempre aponta para trás, ela é constante num conjunto grande.

\subsection{O Princípio}

O princípio $\diamond$ foi introduzido por Ronald Jensen em 1972. Jensen extraiu o Princípio $\diamond$ de sua demonstração que $V=L$ implica na existência de uma árvore de Suslin ${ }^{9}$. Por sinal, uma aplicação que faremos no Capítulo 5A Hipótese de Suslinchapter.5 será que se assumirmos $\diamond$ existirá uma árvore de Suslin.

\footnotetext{
${ }^{8}$ Se $\gamma \in D \cap S$ então para todo $\alpha<\gamma$ implica que $\gamma \in C_{\alpha}$ e então $f(\gamma) \neq \alpha$ pois $C_{\alpha} \cap f^{-1}\{\alpha\} \neq \emptyset$. ${ }^{9}$ Veja [8].
} 
Definição 3.20. $\diamond$ é a seguinte afirmação: Existem conjuntos $A_{\alpha} \subset \alpha$ para $\alpha<\omega_{1}$ tais que: para todo $A \subset \omega_{1}$ temos que o conjunto $\left\{\alpha<\omega_{1}: A \cap \alpha=A_{\alpha}\right\}$ é estacionário. $A$ sequência $\left(A_{\alpha}\right)_{\alpha<\omega_{1}}$ é chamada uma $\diamond$-sequência.

Observe que se tomarmos um $\kappa<\omega_{1}$ então certamente para $\alpha>\kappa$ teremos que $\kappa=\kappa \cap \alpha=A_{\alpha}$. O que devemos observar é que o princípio vale para todo subconjunto $A \subset \omega_{1}$ podendo ter o caso em que $\operatorname{c} f(A)=\omega_{1}$. Mas o conjunto dos $\alpha<\omega_{1}$ onde "o corte $A$ em $\alpha "$ é igual ao termo $A_{\alpha}$ da sequência $\left(A_{\alpha}\right)_{\alpha<\omega_{1}}$ é bem grande (estacionário).

Vamos fechar este capítulo mostrando que o Princípio $\diamond$ é mais forte do que a Hipótese do Contínuo.

Proposição 3.21. $\diamond$ implica $C H$

Demonstração. Se $\left(A_{\alpha}\right)_{\alpha<\omega_{1}}$ é uma $\diamond$-sequência, então para todo $A \subset \omega$ o conjunto $\{\alpha<$ $\left.\omega_{1}: A \cap \alpha=A_{\alpha}\right\}$ é estacionário, e em particular é ilimitado. Assim, existe $\alpha>\omega$ tal que $A=A \cap \alpha=A_{\alpha}$. Portanto $\left\{A_{\alpha}: A_{\alpha} \subset \omega\right\}=\wp(\omega)$. 


\section{Parte II}

Aplicações 



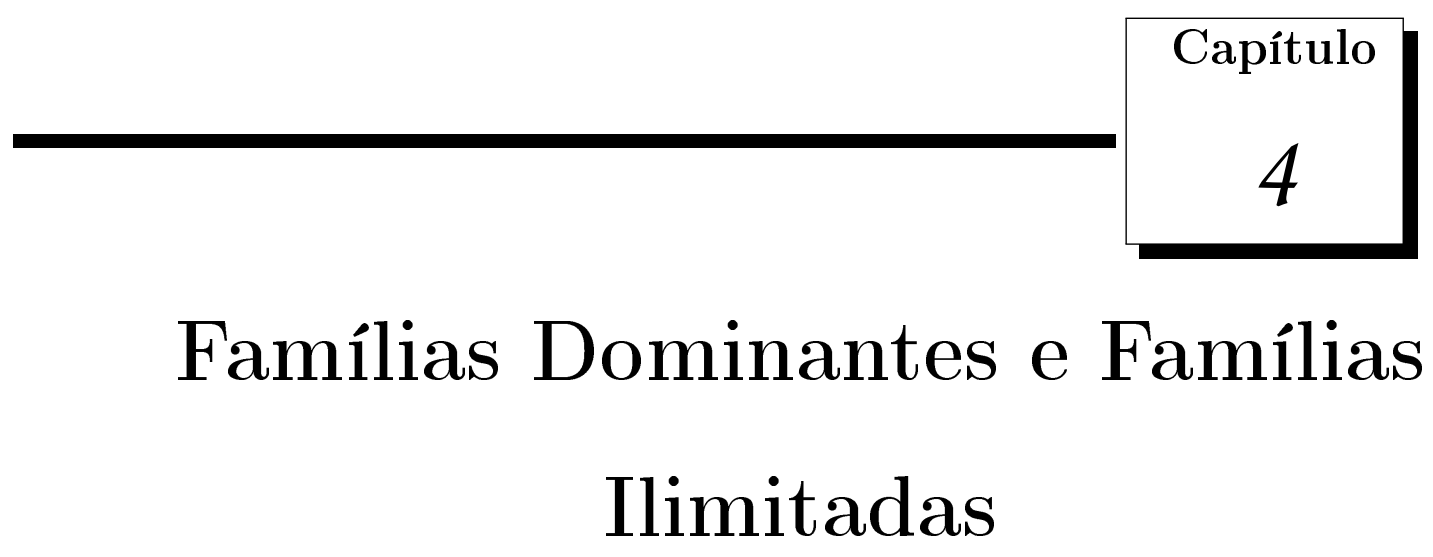

Intuitivamente, podemos entender que uma família $A \subset \omega^{\omega}$ é dominante se para toda $g \in \omega^{\omega}$, existe $f \in A$ tal que $f$ está "acima" de $g$ (onde o "acima" será definido porteriormente). Já uma família $A \subset \omega^{\omega}$ é ilimitada se não existe $g \in \omega^{\omega}$ tal que, para toda $f \in A, g$ está "acima" de $f$. Estudaremos os menores tamanhos possíveis para tais famílias. Nas próximas seções veremos algumas relações entre esses tamanhos, tanto em $Z F C$ como assumindo $M A$.

Esses cardinais tem aplicações interessantes, por exemplo, no Problema de Michael ${ }^{1}$. Ver, por exemplo, [12].

\subsection{Sob $Z F C$}

Nessa seção definiremos tais famílias. Além disso, veremos algumas relações entre os mesmos tamanhos, sem a necessidade de axiomas extras.

Definição 4.1. Sejam $f, g \in \omega^{\omega}$. Dizemos que $f \leq^{*} g$ se $\{n \in \omega: f(n)>g(n)\}$ é finito. Em outras palavras, $f \leq^{*} g$ se existe $k \in \omega$ tal que $f(n) \leq g(n)$ para todo $n>k$.

\footnotetext{
${ }^{1}$ Isto é, se existe um espaço de Lindelöf regular cujo produto com o espaço dos irracionais não seja de Lindelöf.
} 
Mostremos que $\leq *$ é uma pré-ordem (isto é, igual a ordem parcial mas sem a hipótese de que $a \leq b$ e $b \leq a$ implica $a=b$ ).

(1) Seja $f \in \omega^{\omega}$. Como $\{n \in \omega: f(n)>f(n)\}=\emptyset$ então $f \leq^{*} f$ para todo $f \in \omega^{\omega}$.

(2) Sejam $f, g, h \in \omega^{\omega}$ tais que $f \leq^{*} g$ e $g \leq^{*} h$. Então os conjuntos $A=\{n \in$ $\omega: f(n)>g(n)\}$ e $B=\{n \in \omega: g(n)>h(n)\}$ são finitos. Suponha que exista $k \in\{n \in \omega: f(n)>h(n)\}$ tal que $k \notin A$ e $k \notin B$. Então $f(k) \leq g(k)$ e $g(k) \leq h(k)$ que implica $f(k) \leq h(k)$ o que é um absurdo. Logo $\{n \in \omega: f(n)>h(n)\} \subset A \cup B$ que é finito. Portanto $f \leq^{*} h$.

Definição 4.2. Dizemos que uma familia $A \subset \omega^{\omega}$ é ilimitada se não existe $g \in \omega^{\omega}$ tal que, para todo $f \in A, f \leq^{*} g$. Dizemos que uma familia $A \subset \omega^{\omega}$ é dominante se para todo $g \in \omega^{\omega}$, existe $f \in A$ tal que $g \leq^{*} f$.

Com isso temos:

Proposição 4.3. Toda família dominante é ilimitada.

Demonstração. Suponha por absurdo que $A$ seja uma família dominante e limitada, isto é, existe $g \in \omega^{\omega}$ tal que $f \leq^{*} g$ para toda $f \in A$. Defina $\tilde{g}$ como $\tilde{g}(n)=g(n)+1$. Como $A$ é dominante, existe $h \in A$ tal que $\tilde{g} \leq^{*} h$. Como $h \leq^{*} g$ e $\tilde{g} \leq^{*} h$, existe $a \in \omega$ tal que $h(a) \leq g(a)$ e $\tilde{g}(a) \leq h(a)^{2}$. Mas note que, para todo $n \in \omega$, temos que $g(n)<\tilde{g}(n)$. Portanto $h(a)<h(a)$ o que é um absurdo.

Com a próxima proposição, podemos concluir que toda família dominante é não enumerável.

Proposição 4.4. Não existe uma família ilimitada que seja enumerável.

Demonstração. Suponha que exista uma família ilimitada que seja enumerável. Seja $A=$

\footnotetext{
${ }^{2}$ Isso se dá pela definição de $\leq *$.
} 
$\left\{f_{i}\right\}_{i \in \omega}$ tal família. Defina $f$ como:

$$
\begin{aligned}
& f(0)=1+f_{0}(0) \\
& f(1)=1+f_{0}(1)+f_{1}(1) \\
& f(2)=1+f_{0}(2)+f_{1}(2)+f_{2}(2) \\
& \quad \vdots \\
& f(n)=1+\sum_{i=0}^{n} f_{i}(n) .
\end{aligned}
$$

Note que até pode occorrer que $f_{1}(0)>f(0)$, mas $f_{1}(n)<f(n)$ para todo $n \geq 1$. Logo $\left|\left\{n \in \omega: f_{1}(n)>f(n)\right\}\right| \leq 1$. Assim como pode ocorrer que $f_{2}(0)>f(0)$ e $f_{2}(1)>f(1)$, porém $f_{2}(n)<f(n)$ para $n \geq 2$. Então $\left|\left\{n \in \omega: f_{2}(n)>f(n)\right\}\right| \leq 2$. Assim, para $k \in \omega$ :

$$
\left|\left\{n \in \omega: f_{k}(n)>f(n)\right\}\right| \leq k .
$$

$\operatorname{Logo} f_{k} \leq^{*} f$. Portanto $A$ é limitada.

Agora, definiremos os números cardinais $\mathfrak{d}$ e $\mathfrak{b}$ e veremos algumas relações, entre eles sob $Z F C$. Na próxima seção, veremos o que acontece sob $M A$.

Definição 4.5. Chamamos de $\mathfrak{d}$ o menor cardinal tal que exista uma família dominante de tal cardinalidade. Chamamos de $\mathfrak{b}$ o menor cardinal tal que exista uma família ilimitada de tal cardinalidade.

Então, temos uma relação entre $\mathfrak{d}$ e $\mathfrak{b}$ em ZFC:

Teorema 4.6. $\omega<\mathfrak{b} \leq \mathfrak{d} \leq 2^{\omega}$.

Demonstração. Note que $\omega^{\omega}$ é tanto uma uma família dominante como uma família ilimitada e, por $\left|\omega^{\omega}\right|=2^{\omega}$, segue que $\mathfrak{b} \leq 2^{\omega}$ e $\mathfrak{d} \leq 2^{\omega}$. Como toda família dominante é ilimitada não podemos ter que $\mathfrak{d}<\mathfrak{b}$, então $\mathfrak{b} \leq \mathfrak{d}$. Como não existe família ilimitada enumerável segue que $\omega<\mathfrak{b}$. Portanto $\omega<\mathfrak{b} \leq \mathfrak{d} \leq 2^{\omega}$.

Observe que se assumirmos $C H$ então $\mathfrak{b}=\mathfrak{d}=2^{\omega}\left(=\omega_{1}\right)$. 


\subsection{Sob o Axioma de Martin}

Na seção anterior mostramos a seguinte estimativa $\omega<\mathfrak{b} \leq \mathfrak{d} \leq 2^{\omega}$. Assumindo $M A$ concluiremos que $\mathfrak{b}=\mathfrak{d}=2^{\omega}$. Para isso, considere a seguinte definição:

Definição 4.7. Seja $\left(f_{\xi}\right)_{\xi<\kappa}$ uma familia de funções de $\omega$ em $\omega$, onde $\kappa<2^{\omega}(\omega<\kappa)$. Considere o conjunto $\mathbb{P}=\left\{(s, F): s \in \omega^{<\omega}, F \subset \kappa\right.$ finito $\}$. Defina em $\mathbb{P}$ a ordem

$$
\begin{gathered}
(s, F) \leq(t, G) \text { se, e somente se, } s \supset t \text { e } F \supset G \text { e, para todo } \alpha \in G \text { e para todo } \\
n \in d o m s \backslash d o m t \text {, temos } s(n)>f_{\alpha}(n) .
\end{gathered}
$$

Com isso, temos que:

(1) $\leq$ é uma ordem sobre $\mathbb{P}$. De fato:

(i) $(s, F) \leq(s, F):$ Seja $(s, F) \in \mathbb{P}$ então $s \supset s$ e $F \supset F$ e $\operatorname{dom} s \backslash \operatorname{dom} s=\emptyset$;

(ii) Se $(s, F) \leq(t, G)$ e $(t, G) \leq(s, F)$, então $(s, F)=(t, G)$ : Como $s \supset t, F \supset G$ e $t \supset s, G \supset F$, então $s=t$ e $F=G$. Logo, $(s, F)=(t, G)$;

(iii) Se $(s, F) \leq(t, G)$ e $(t, G) \leq(f, H)$ então $(s, F) \leq(f, H)$ : Temos que $s \supset t$, $F \supset G, t \supset s$ e $G \supset H$. Então $s \supset f$ e $F \supset H$. Note que $\operatorname{dom} s \backslash \operatorname{dom} f=$ $(\operatorname{dom} s \backslash \operatorname{dom} t) \cup(\operatorname{dom} t \backslash \operatorname{dom} f)$. Se $n \in \operatorname{dom} s \backslash \operatorname{dom} t$, então $s(n)>f_{\alpha}(n)$ para todo $\alpha \in G$. Em particular, para todo $\alpha \in H$, pois $H \subset G$. Se $n \in \operatorname{dom} t \backslash \operatorname{dom} f$, então $t(n)>f_{\alpha}(n)$ para $\alpha \in H$ e então $s(n)>f_{\alpha}(n)$, pois $s \supset t$. Portanto $(s, F) \leq(f, H)$.

(2) $(\mathbb{P}, \leq)$ satisfaz c.c.c.

Lembrando que: $A \subset \mathbb{P}$ é anticadeia se dados $(s, F),(t, G) \in A$ distintos não existe $(f, H) \in \mathbb{P}$ tal que $(f, H) \leq(s, F),(t, G)$.

Seja $A \subset \mathbb{P}$ uma anticadeia não-enumerável. Note que $\omega^{<\omega}=\bigcup_{n \in \omega} \omega^{n}$ é enumerável, então pelo menos uma das funções $s$ dos pares $(s, F)$ em $A$ vai se repetir. Seja $s^{\prime}$ uma função que se repete. Considere $\left(s^{\prime}, F^{\prime}\right),\left(s^{\prime}, F^{\prime \prime}\right) \in A$ e defina $F=F^{\prime} \cup F^{\prime \prime}$. Tome $\left(s^{\prime}, F\right) \in \mathbb{P}$ e note que $F \supset F^{\prime}, F^{\prime \prime}$. Portanto $\left(s^{\prime}, F\right) \leq\left(s^{\prime}, F^{\prime}\right),\left(s^{\prime}, F^{\prime \prime}\right)$. Com isso contrariamos o fato de $A$ ser anticadeia. 
(3) Para cada $\xi<\kappa, D_{\xi}=\{(s, F) \in \mathbb{P}: \xi \in F\}$ é denso em $(\mathbb{P}, \leq)$.

De fato, seja $(s, F) \in \mathbb{P}$ e seja $F^{\prime}=F \cup\{\xi\}$ então $\left(s, F^{\prime}\right) \in D_{\xi}$ e $\left(s, F^{\prime}\right) \leq(s, F)$.

(4) Para cada $n<\omega, A_{n}=\{(s, F) \in \mathbb{P}: n \in \operatorname{dom} s\}$ é denso em $(\mathbb{P}, \leq)$.

De fato, seja $(s, F) \in \mathbb{P}$. Se $n \in \operatorname{dom} s$ então $(s, F) \in A_{n}$.

Se $n \notin$ dom $s$ consideremos o caso particular em que $\operatorname{dom} s=n-1$ e considere $k_{n}=$ $\max _{\alpha \in F}\left\{f_{\alpha}(n)\right\}+1$. Então, defina $t(a)=s(a)$ se $a<n$ e $t(n)=k_{n}$. Assim, para todo $\alpha \in F$ temos que $t(n)>f_{\alpha}(n)$ e então $(t, F) \leq(s, F)$. Agora, se $\operatorname{dom} s=m<n$, basta construir $k_{j}:=\max _{\alpha \in F}\left\{f_{\alpha}(j)\right\}+1$ para $m<j \leq n$ e definir

$$
t(a)= \begin{cases}s(a) & \text { se } a<m, \\ k_{m} & \text { se } a=m, \\ k_{m+1} & \text { se } a=m+1, \\ \vdots & \\ k_{n} & \text { se } a=n .\end{cases}
$$

Portanto $(t, F) \leq(s, F)$.

(5) O Axioma de Martin implica que $\left(f_{\xi}\right)_{\xi<\kappa}$ acima fixada não é ilimitada.

Em primeiro lugar, observe que a família $\left\{A_{n}: n \in \omega\right\} \cup\left\{D_{\xi}: \xi<\kappa\right\}$ é formada por conjuntos densos em $\mathbb{P} \operatorname{com} \xi<\kappa$ e $n<\omega$ e que possui $\kappa$ elementos ${ }^{3}$. Portanto, pelo Axioma de Martin, existe um filtro $G \subset \mathbb{P}$ tal que, para todo $\xi<\kappa, G \cap D_{\xi} \neq \emptyset$ e para todo $n<\omega, G \cap A_{n} \neq \emptyset$.

Seja $f=\bigcup_{(s, F) \in G} s$ e observe que:

(i) $f$ é função: Suponha que existam $(n, k),\left(n, k^{\prime}\right) \in f$ com $k \neq k^{\prime}$. Então existem $(s, F),(t, H) \in G$ tal que $(n, k) \in s$ e $\left(n, k^{\prime}\right) \in t$. Como $G$ é filtro existe $(p, A) \leq$ $(s, F),(t, H) \operatorname{com}(p, A) \in G$ e, assim, $p \supset s$ e $p \supset t$. Então $(n, k),\left(n, k^{\prime}\right) \in p$ e por $p$ ser função segue que $k=k^{\prime}$.

(ii) $\operatorname{dom} f=\omega$ : Isso se dá pelo fato de que para todo $n<\omega$ termos $G \cap A_{n} \neq \emptyset$.

\footnotetext{
${ }^{3}$ Pois $\kappa$ é não enumerável enquanto $\omega$ é enumerável.
} 
(iii) $\left\{n \in \omega: f_{\xi}(n)>f(n)\right\}$ é finito para todo $\xi<\kappa$. Para isso vamos mostrar que existe $(s, F) \in G \cap D_{\xi}$ tal que o conjunto $\left\{n \in \omega: f_{\xi}(n)>f(n)\right\} \subset F \cup \operatorname{dom} s$. Seja $(s, F) \in G \cap D_{\xi}$ e suponha que exista $k \in\left\{n \in \omega: f_{\xi}(n)>f(n)\right\}$ tal que $k \notin F \cup$ doms. Seja $(t, H) \in G \cap A_{k+1}$, como $G$ é filtro existe $(g, A) \in G$ tal que $(g, A) \leq(s, F),(t, H)$. Então, para todo $\alpha \in F$ e para todo $n \in \operatorname{dom} g \backslash \operatorname{dom} s$, temos que $g(n)>f_{\alpha}(n)$ e, em particular, $f(k)=g(k)>f_{\xi}(k)$. O que é um absurdo.

Acabamos de ver que, para uma família de funções $\left(f_{\xi}\right)_{\xi<\kappa}$ onde $\kappa<2^{\omega}$ se assurmirmos $M A$ tal família não poderá ser ilimitada. Tendo isso em mente, temos:

Teorema 4.8. O Axioma de Martin implica que $\mathfrak{b}=\mathfrak{d}=2^{\omega}$.

Demonstração. Note que $\omega^{\omega}$ é tanto uma uma família dominante como uma família ilimitada e $\left|\omega^{\omega}\right|=2^{\omega}$. Como não existe família ilimitada de cardinalidade $\kappa<2^{\omega}$ e toda família dominante é ilimitada o resultado segue. 


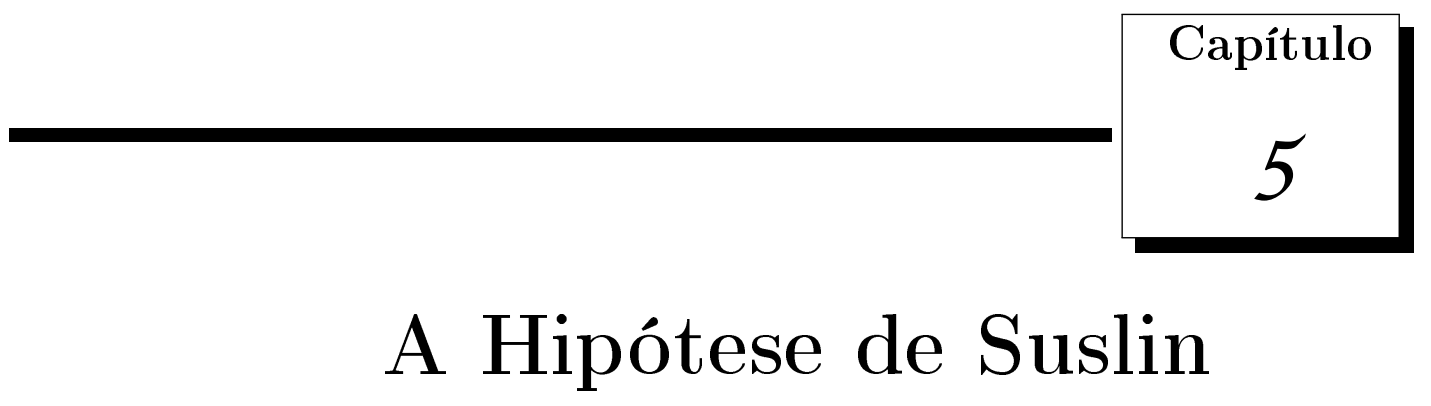

Consideremos a reta $(\mathbb{R},<)$ e listemos algumas propriedades:

(1) $(\mathbb{R},<)$ é totalmente ordenado,

(2) $(\mathbb{R},<)$ não possui elemento minimal e maximal,

(3) $(\mathbb{R},<)$ é conexo e é separável na topologia da ordem.

Existe algum outro conjunto $(X, \prec)$ diferente de $(\mathbb{R},<)$ que compartilha das mesmas propriedades que acabamos de dizer? Respondendo a pergunta (e estragando a surpresa!) na primeira seção mostraremos que um conjunto $(X, \prec)$ com tais propriedades precisa ser isomorfo $^{1}$ à $(\mathbb{R},<)$.

Agora, o que acontece se substituirmos a condição de separabilidade por satisfazer a c.c.c.? Será que isso também caracteriza $(\mathbb{R},<)^{2}$ ? O Teorema 7.1 Produto de espaços c.c.cteorema.7.1 nos diz que se um espaço topológico é separável então satisfaz a c.c.c., mas, em geral, a recíproca não é verdadeira. Será que existe um conjunto $(X, \prec)$ totalmente ordenado, sem elemento maximal e minimal, conexo na topologia da ordem, c.c.c. e que não seja separável? Ou será que isso também é outro tipo de caracterização para $(\mathbb{R},<)$ ?

\footnotetext{
${ }^{1}$ Neste capítulo o isomorfiso é no sentido de ordem, i.e. existe uma função bijetora que preserva a ordem.

${ }^{2}$ Lembrando que $(\mathbb{R},<)$ satisfaz a c.c.c..
} 
Para isso temos uma resposta curiosa, a existência de tal conjunto é independente de $Z F C$ ! Esse conjunto é conhecido como reta de Suslin. Formalmente chamamos de Reta de Suslin um conjunto totalmente ordenado $(X,<)$ com a topologia da ordem, onde:

(1) $X$ não possui elemento maximal nem minimal;

(2) $X$ é conexo na topologia da ordem;

(3) X satisfaz c.c.c, mas não é separável.

Além disso, chamamos de Hipótese de Suslin $(S H)$ a afirmação "não existem retas de Suslin".

Provaremos a independência de $S H$ mostrando na segunda seção que $\diamond$ implica a negação de $S H$ e, na seção seguinte, mostraremos que $M A$ implica $S H$ (mostraremos também que $M A$ implica $S H$ no capítulo 7 Produto de espaços c.c.cchapter.7 de uma maneira diferente).

Então, respire fundo e se prepare, pois o capítulo é um pouco extenso e bem técnico.

\subsection{Motivação}

Mostremos que qualquer conjunto totalmente ordenado $(X,<)$ satisfazendo:

(1) $X$ não possui elemento maximal nem minimal;

(2) $X$ é conexo na topologia da ordem;

(3) X é separável na topologia da ordem.

é isomorfo (no sentido de ordem) à $(\mathbb{R},<)$.

Para isso considere o seguinte Lema:

Lema 5.1. Seja $(D,<)$ um conjunto enumerável, totalmente ordenado, que não possua elemento minimal e nem maximal e que seja denso sobre si mesmo, i.e., para todo $x, y$ com $x<y$ temos que existe z tal que $x<z<y$. Então $D$ é isomorfo aos racionais.

Demonstração. Seja $D=\left\{d_{n}: n \in \omega\right\}$ e $\mathbb{Q}=\left\{q_{n}: n \in \omega\right\}$. Vamos definir $f: D \rightarrow \mathbb{Q}$ por indução da seguinte maneira: 
- $f\left(d_{0}\right)=q_{0}$;

- Seja $d_{1} \in D$, então $d_{0}<d_{1}$ ou $d_{1}<d_{0}$.

Se $d_{0}<d_{1}$ considere $A_{1}=\left\{n \in \omega: q_{0}<q_{n}\right\}$. Note que $A_{1} \neq \emptyset$, pois $\mathbb{Q}$ não possui elemento maximal. Como $\omega$ é bem ordenado, $A_{1}$ possui um elemento mínimo e seja $n_{1}$ tal elemento. Defina $f\left(d_{1}\right)=q_{n_{1}}$.

Se $d_{1}<d_{0}$ considere $B_{1}=\left\{n \in \omega: q_{n}<q_{0}\right\}$ e, de maneira análoga, obtenha $n_{1} \mathrm{e}$ defina $f\left(d_{1}\right)=q_{n_{1}}$.

Seja $d_{2} \in D$. Digamos que $d_{0}<d_{1}$ (para $d_{1}<d_{0}$ a construção é análoga) então:

- Se $d_{1}<d_{2}$, considere $A_{2}=\left\{n \in \omega: q_{n_{1}}<q_{n}\right\}$. Novamente $A_{2} \neq \emptyset$, seja $n_{2}$ o elemento mínimo de $A_{2}$. Defina $f\left(d_{2}\right)=q_{n_{2}}$.

- Se $d_{2}<d_{0}$, considere $B_{2}=\left\{n \in \omega: q_{n}<q_{0}\right\}$ e de maneira análoga obtenha $n_{2}$ e defina $f\left(d_{2}\right)=q_{n_{2}}$.

- Se $d_{0}<d_{2}<d_{1}$, considere $C_{2}=\left\{n \in \omega: q_{0}<q_{n}<q_{n_{1}}\right\}$. Temos que $C_{2} \neq \emptyset$, pois $\mathbb{Q}$ é denso em si mesmo. Então, seja $n_{2}$ o elemento mínimo de $C_{2}$ e defina $f\left(d_{2}\right)=q_{n_{2}}$.

Agora, suponha que já tenhamos determinado $f\left(d_{s}\right)=q_{n_{s}}$ para todo $s<k$. Determinaremos $q_{k}$ para $d_{k} \in D$. Vamos analisar os três casos possíveis:

(1) Se $\max _{s<k}\left\{d_{s}\right\}<d_{k}$. Considere $A_{k}=\left\{n \in \omega: \max _{s<k}\left\{q_{n_{s}}\right\}<q_{n}\right\}$. Temos que $A_{k} \neq \emptyset$, pois $\mathbb{Q}$ não tem elemento maximal. Seja $n_{k}$ o mínimo do conjunto $A_{k} \mathrm{e}$ defina $f\left(d_{k}\right)=q_{n_{k}}$.

(2) Se $d_{k}<\min _{s<k}\left\{d_{s}\right\}$. Considere $B_{k}=\left\{n \in \omega: q_{n}<\min _{s<k}\left\{q_{n_{s}}\right\}\right.$, de maneira análoga obtenha $n_{k}$ e defina $f\left(d_{k}\right)=q_{n_{k}}$.

(3) Se $d_{a}<d_{k}<d_{b}$, onde $a, b<k$. Sejam $q_{n_{a}}=f\left(d_{a}\right)$ e $q_{n_{b}}=f\left(d_{b}\right)$. Considere $C_{k}=\left\{n \in \omega: q_{n_{a}}<q_{n}<q_{n_{b}}\right\}$. Novamente $C_{k} \neq \emptyset$ pois $\mathbb{Q}$ é denso em si mesmo. Seja $n_{k}$ o mínimo de $C_{k}$ e defina $f\left(d_{k}\right)=q_{n_{k}}$.

Portanto $f: D \rightarrow \mathbb{Q}$ está bem definida por indução e:

- $f$ preserva a ordem; 
- $f$ é injetora;

- $f$ é sobrejetora.

De fato, suponha que $f$ não seja sobrejetora. Seja $Q=\left\{n \in \omega: q_{n} \neq f(d)\right.$, para todo $d \in$ $D\}$. Então existe $k$, tal que $k$ é o mínimo de $Q$. Observe que, para todo $s<k, q_{s}=f\left(d_{m_{s}}\right)$ para algum $d_{m_{s}} \in D$. A idéia, agora, é "completar" o domínio para analisar a distribuição das imagens desses pontos. Como $\left\{d_{m_{s}}: s<k\right\}$ é finito seja $d_{m}^{\prime}=\max _{s<k}\left\{d_{m_{s}}\right\}$ e seja $d_{m}^{\prime \prime}=\min _{s<k}\left\{d_{m_{s}}\right\}$ e considere $Z=\left\{q_{m_{s}}=f\left(d_{m_{s}}\right): d_{m}^{\prime \prime}<d_{m_{s}}<d_{m}^{\prime}\right\}$.

Considere os seguintes casos:

(i) $q_{m_{s}}<q_{k}$ para todo $q_{m_{s}} \in Z$. Seja $\ell$ o menor índice tal que exista $d_{\ell} \in D$ com $d_{m}^{\prime}<d_{\ell}$. Então $f\left(d_{\ell}\right)=q_{k}$, pois $k$ é o menor índice tal que $q_{m_{s}}<q_{k}$ para todo $q_{m_{s}} \in Z$.

(ii) $q_{k}<q_{m_{s}}$ para todo $q_{m_{s}} \in Z$. De maneira análoga existe $d_{\ell} \in D$ tal que $f\left(d_{\ell}\right)=q_{k}$.

(iii) $q_{k}$ está entre dois elementos de $Z$ Sejam $q_{a}, q_{b} \in Z$ tal que $q_{a}<q_{k}<q_{b}$. Logo existem $d_{m_{a}}, d_{m_{b}} \in D$ tal que $f\left(d_{m_{a}}\right)=q_{a}$ e $f\left(d_{m_{b}}\right)=q_{b}$. Seja $W=\left\{n \in \omega: d_{m_{a}}<\right.$ $\left.d_{n}<d_{m_{b}}\right\}$. Como $D$ é denso nele mesmo então $W \neq \emptyset$ e $W \subset \omega$. Seja $\ell$ o mínimo de $W$, então $f\left(d_{\ell}\right)=q_{k}$.

Com isso, agora estamos preparados para mostrar o que foi proposto no início da seção.

Teorema 5.2. Seja $(X,<)$ totalmente ordenado satisfazendo:

(1) X não possui elemento maximal e nem minimal;

(2) X é conexo na topologia da ordem;

(3) X é separável na topologia da ordem.

Então $(X,<)$ é isomorfo a $(\mathbb{R},<)$ 
Demonstração. Seja $D$ um subconjunto denso e enumerável em $X$ (na topologia da ordem $)^{3}$. Considere $(D,<)$ onde " $<$ " é a ordem induzida por $(X,<)$. Logo $(D,<)$ é totalmente ordenado. Observe que:

- $D$ não possui elemento maximal e minimal. Suponha que exista $d \in D$ maximal e por (1) podemos tomar $x_{1}, x_{2} \in X$ tal que $d<x_{1}<x_{2}$, disso concluimos que $x_{1}, x_{2} \in X \backslash D$. Logo $] d, x_{2}\left[\neq \emptyset\right.$, pois $\left.x_{1} \in\right] d, x_{2}\left[\right.$. Como $D$ é denso existe $d^{\prime} \in D$ tal que $\left.d^{\prime} \in\right] d, x_{2}\left[\right.$. Logo $d<d^{\prime}$ o que é uma contradição. Para mostrar que não existe elemento minimal o raciocício é análogo.

- $D$ é denso sobre si mesmo. De fato, sejam $d_{1}, d_{2} \in D$ com $d_{1}<d_{2}$. Se $] d_{1}, d_{2}[\neq \emptyset$ então como $D$ é denso em $X$ existe $z \in D$ tal que $z \in] d_{1}, d_{2}$ [ isto é, $d_{1}<z<d_{2}$. Agora, suponha que $] d_{1}, d_{2}\left[=\emptyset\right.$, então $\left\{x \in X: x<d_{1}\right\} \neq \emptyset,\left\{x \in X: d_{2}<x\right\} \neq \emptyset$ e $X=\left\{x \in X: x<d_{1}\right\} \cup\left\{x \in X: d_{2}<x\right\}$ o que contraria (2).

Logo $(D,<)$ está nas condições do lema anterior. Seja $g:(\mathbb{Q},<) \rightarrow(D,<)$ um isomorfismo (no sentido de ordem). Vamos definir $\tilde{g}:(\mathbb{R},<) \rightarrow(X,<)$ da seguinte maneira:

- Se $x \in \mathbb{Q}$, então $\tilde{g}(x)=g(x)$;

- Se $x \in \mathbb{R} \backslash \mathbb{Q}$. Seja $A_{x}=\{q \in \mathbb{Q}: q<x\}$ então $A_{x} \neq \emptyset$ e $\sup A_{x}=x$. Considere $B_{\tilde{g}(x)}=\{g(q): q \in \mathbb{Q}, q<x\}$. Note que $B_{\tilde{g}(x)} \neq \emptyset$. Defina $\tilde{g}(x)=\sup B_{\tilde{g}(x)}{ }^{4}$.

Observações:

(i) $\tilde{g}$ preserva ordem (e portanto $\tilde{g}$ é injetora). Sejam $x, y \in \mathbb{R}$ tal que $x<y$. Logo existe $z_{1} \in \mathbb{Q}$ tal que $x<z_{1}<y$ e disso existe $z_{2} \in \mathbb{Q}$ tal que $x<z_{1}<z_{2}<y$. Logo $\sup B_{\tilde{g}(x)} \leq g\left(z_{1}\right)<g\left(z_{2}\right) \leq \sup B_{\tilde{g}(y)}$ e então $\tilde{g}(x)<\tilde{g}(y)$. Portanto $\tilde{g}(x) \neq \tilde{g}(y)$.

(ii) $\tilde{g}$ é sobrejetora. Seja $a \in X \backslash D$. Seja $A=\{d \in D: d<a\}$, note que $\sup A=a$. Seja $B=\{q \in \mathbb{Q}: g(q)<a\}$, e note também que $B \neq \emptyset$ e limitado ${ }^{5}$. Então existe $\sup B$. Defina $b=\sup B$.

\footnotetext{
${ }^{3}$ Isso é possível por (3).

${ }^{4}$ Isso é possível pela Proposição 2.8Noções Básicas de Topologiateorema.2.8

${ }^{5}$ Pois existe $d \in D$ tal que $a<d$ e $q<g^{-1}(d)$ para todo $q \in B$.
} 
Afirmamos que $\tilde{g}(b)=a$. Vamos mostrar que $\sup A=\sup C_{\tilde{g}(b)}$, onde $C_{\tilde{g}(b)}=$ $\{g(q) \in D: q<b\}$. Suponha que $\sup A<\sup C_{\tilde{g}(b)}$. Então existe $\tilde{q} \in \mathbb{Q} \operatorname{com} \tilde{q}<b$ tal que $d<g(\tilde{q})$ para todo $d \in A$, mas $\tilde{q} \in B$, $\operatorname{logo} g(\tilde{q})<a$ e então $g(\tilde{q}) \in A$. Suponha que $\sup C_{\tilde{g}(b)}<\sup A$. Então existe $\tilde{d} \in D \operatorname{com} \tilde{d}<a$ tal que $g(q)<\tilde{d}$ para todo $g(q) \in C_{\tilde{g}(b)}$, mas $g^{-1}(\tilde{d})<b$ então $\tilde{d} \in C_{\tilde{g}(b)}$. Portanto $\sup A=\sup C_{\tilde{g}(b)}$.

\section{$5.2 M A$ implica $S H$}

Mostraremos que $M A$ implica $S H$ em duas etapas: na primeira, mostraremos que assumindo $M A$ não existe uma árvore de Suslin. Na segunda etapa, mostraremos, que a existência de uma reta de Suslin é equivalente à existência de uma árvore de Suslin.

Teorema $5.3(M A)$. Não existe uma árvore de Suslin.

Demonstração. Seja $(T, \leq)$ uma árvore satisfazendo as condições do Corolário 1.45Árvoresteorema.1.45 e seja $\left(A_{\alpha}\right)_{\alpha<\omega_{1}}$ onde $A_{\alpha}=\{s \in T: h(s) \geq \alpha\}$. Vamos mostrar que cada $A_{\alpha}$ é denso em $T$ na ordem reversa ${ }^{6}$. Seja $t \in T$. Então novamente pelo Corolário 1.45Árvoresteorema.1.45, existe $s \in T$ tal que $s>t \operatorname{com} h(s)=\beta$ onde $h(t)<\beta<\omega_{1}$. Se $\beta>\alpha$ então $s \in A_{\alpha}$, senão aplicamos novamente o corolário em $s$ até obter $s^{\prime}>s \operatorname{com} h\left(s^{\prime}\right) \geq \alpha$. Então $s \in A_{\alpha}$ e $t \leq s$. Como $T$ satisfaz c.c.c. e cada $A_{\alpha}$ é denso em $T$ segue do Axioma de Martin que existe $F \subset T$ filtro tal que $F \cap A_{\alpha} \neq \emptyset$. Como os elementos de $F$ são compatíveis na ordem reversa segue que são comparáveis na ordem usual, então temos um ramo de comprimento $\omega_{1}$ que contradiz a definição de árvore de Suslin.

Agora o nosso objetivo é mostrar que a existência de uma reta de Suslin é equivalente à existência de uma Árvore de Suslin.

Teorema 5.4. Dada uma reta de Suslin $\left(Y,<_{Y}\right)$, então existe uma reta de Suslin $X$ tal que:

(1) $X$ é denso em si mesmo ${ }^{7}$;

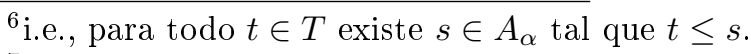

7 i.e., se $a<b$ então $] a, b[\neq \emptyset$
} 
(2) Nenhum subconjunto aberto de $X$ é separável.

Demonstração. Seja $Y$ uma reta de Suslin. Defina uma relação “ " em $Y$ dada por: $x \sim y$ se, e somente se, o intervalo entre esses pontos (]x, $y[$ se $x \leq y$ ou $] y, x[$ se $y \leq x)$ é separável.

Observe que " " é uma relação de equivalência. De fato:

(i) $x \sim x$, pois $\emptyset$ é separável;

(ii) $x \sim y$ se, e somente se, $y \sim x$ pela definição de “ ";

(iii) Se $x \sim y$ e $y \sim z$ então $y \sim z$. De fato, pois se $] x, y[$ e $] y, z[$ são separáveis, então ]$x, z[=] x, y[\cup\{y\} \cup] y, z[$ é separável.

Seja $X$ o conjunto das classes de equivalência.

Note que se $I \in X$ então $I$ é convexo em $Y^{8}$. De fato, suponha que existam $a, b \in I$ tais que que $] a, b[\nsubseteq I$. Ou seja existe $c \in] a, b[$ tal que $c \notin I$. Seja $J \in X$ tal que $J \neq I$ e $c \in J$. Como $a, b \in I$ então $a \sim b$ e assim $] a, b[$ é separável. Pelo Lema 2.24Noções Básicas de Topologiateorema.2.24 segue que ]a,c[é separável e, portanto, $a \sim c$ implica que $I=J$ o que é um absurdo. Portanto $] a, b[\subset I$.

Para $I, J \in X$, defina $I<J$ se, e somente se existem $x \in I$ e $y \in J$ tal que $x<y$. Vamos mostrar que < está bem definida e que é uma ordem total:

- Pela observação anterior, para $x, y \in I$ temos $] x, y[\subset I$, com isso vemos que $<$ está bem definido, ou seja que $I$ é convexo em $Y$.

- Seja $I \in X$. Então não vale $I<I$;

- A antissimetria e a transitividade seguem trivialmente;

- $<$ é ordem total. De fato, sejam $I \neq J \in X$ quaisquer, logo existem $x \in I$ e $y \in J$ tal que $x \nsim y$, em particular $x \neq y$. Como $Y$ é totalmente ordenado segue que ou $x<_{Y} y$ ou $y<_{Y} x$.

\footnotetext{
${ }^{8}$ i.e. se $x, y \in I$ e $x<y$ então $] x, y[\subset I$.
} 
Note que cada $I \in X$ é separável. Para ver isso, seja $\mathcal{M}$ uma coleção maximal de intervalos não vazios dois a dois disjuntos da forma $] x, y[\operatorname{com} x, y \in I$. Observe que $\mathcal{M}$ é enumerável, pois $Y$ é c.c.c.. Seja $\mathcal{M}=\{] x_{n}, y_{n}[: n \in \omega\}$. Como $x_{n} \sim y_{n}$, seja $D_{n}$ um subconjunto denso enumerável de $] x_{n}, y_{n}\left[\right.$. Seja $D=\bigcup_{n<\omega} D_{n}$, então $D$ é denso em $\left.\bigcup_{n<\omega}\right] x_{n}, y_{n}[$. De fato, seja $] x, y[\subset I$ não vazio, então $] x, y$ [intercepta algum $] x_{n}, y_{n}$ [ pela maximalidade de $\mathcal{M}$. Como $] x, y[\cap] x_{n}, y_{n}$ [ é aberto não vazio, $] x, y[\cap] x_{n}, y_{n}[$ intercepta $D_{n}$. Portanto $\bigcup_{n} D_{n}$ é denso em $I$.

Para ver que $X$ é denso em si mesmo, suponha que existam $I, J \in X$ tal que $I<J$ com $] I, J[=\emptyset$. Seja $x \in I$ e $y \in J$ daí $] x, y[\subset I \cup J$. Como $I, J$ são separáveis segue que $I \cup J$ é separável, assim $x \sim y, \operatorname{logo} I=J$ o que é um absurdo.

Para verificar (2) é suficiente ver que $] I, J[$ não é separável sempre que $I<J$, pois ]$I, J[$ é um aberto básico da topologia da ordem de $(X,<)$. Então, suponha que existam $I, J \in X \operatorname{com} I<J$ tal que $] I, J\left[\right.$ seja separável e seja $\left\{K_{n}: 2 \leq n<\omega\right\}$ um subconjunto denso e enumerável em $] I, J\left[\right.$, e seja $K_{0}=I$ e $K_{1}=J$. Em $Y$, seja $D_{n}$ um subconjunto enumerável e denso em $K_{n}$, então $\bigcup_{n} D_{n}$ é denso em $\bigcup_{I \leq L \leq J} L$ em relação à $\left(Y,<_{Y}\right)$. De fato, seja $] x, y[$ aberto não vazio tal que $] x, y\left[\subset \bigcup_{I \leq L \leq J} L\right.$. Então:

- Se $] x, y\left[\subset L\right.$ para algum $I \leq L \leq J$, então se $L=k_{n}$ para algum $n \in \omega$ teremos que ]$x, y\left[\cap D_{n} \neq \emptyset\right.$ e então $] x, y[\cap D \neq \emptyset$.

- Agora, seja $] x, y\left[\operatorname{com} x \in L_{x}\right.$ e $y \in L_{y}$ para alguns $\left.L_{x}, L_{y} \in\right] I, J[$ distintos. Observe que $] L_{x}, L_{y}[\neq \emptyset$, pois caso contrário, $] x, y\left[\subset L_{x} \cup L_{y}\right.$ e com isso $L_{x}=L_{y}$. Então ] $L_{x}, L_{y}\left[\right.$ intercepta $K_{m}$ para algum $m \in \omega$, ou seja, $L_{x}<K_{m}<L_{y}$ [. Seja $D_{m} \subset K_{m}$, então $] x, y\left[\cap D_{m} \neq \emptyset\right.$.

Com isso concluimos que $\bigcup_{I \leq L \leq J} L$ é separável. Logo, se $x \in I$ e $y \in J$ temos que ]$x, y\left[\subset \bigcup_{I \leq L \leq J} L\right.$. Pelo Lema 2.24Noções Básicas de Topologiateorema.2.24 temos que ] $x, y$ [ é separável e portanto $x \sim y$ (i.e., $I=J$ ) o que é uma contradição.

Finalmente, para mostrar que $X$ é c.c.c. suponha que \{]$I_{\alpha}, J_{\alpha}\left[: \alpha<\omega_{1}\right\}$ seja uma anticadeia. Tome $x_{\alpha} \in I_{\alpha}$ e $y_{\alpha} \in J_{\alpha}$, note que cada $] x_{\alpha}, y_{\alpha}[\neq \emptyset$, pois cada $] x_{\alpha}, y_{\alpha}[$ não é separável ${ }^{9}$. Para $\alpha<\beta<\omega_{1}$ temos que $] x_{\alpha}, y_{\alpha}[\cap] x_{\beta}, y_{\beta}[=\emptyset$, caso contrário,

\footnotetext{
${ }^{9} \emptyset$ é separável.
} 
tome $z \in] x_{\alpha}, y_{\alpha}[\cap] x_{\beta}, y_{\beta}\left[\right.$ e assim $z \in I$ para algum $I_{\alpha}<I<J_{\alpha}$ e $z \in I^{\prime}$ para algum $I_{\beta}<I^{\prime}<J_{\beta}$. Sejam $a \in I$ e $a^{\prime} \in I^{\prime} \operatorname{com} a<z<a^{\prime}$ e observe que $] a, z[\subset I$ é separável e $] z, a^{\prime}\left[\subset I^{\prime}\right.$ é separável. Portanto, $] a, z[\cup\{z\} \cup] z, a^{\prime}\left[\subset I \cup I^{\prime}\right.$ é separável e assim $I=I^{\prime}$. Logo $\left\{\left(x_{\alpha}, y_{\alpha}\right): \alpha<\omega_{1}\right\}$ é uma anticadeia em $Y$. Absurdo.

Portanto $X$ é c.c.c..

Vamos agora mostrar a equivalência da existência de uma reta de Suslin com a existência de uma árvore de Suslin. Para isso, considere o seguinte Lema auxiliar:

Lema 5.5. Seja $(T, \leq)$ uma árvore bem podada de altura $\omega_{1}$ tal que todo ramo tem comprimento enumerável. Para todo $x \in T$ vale a seguinte afirmação:

para todo $n<\omega$ existe $\alpha>h(x)$ tal que $\left|\left\{y \in \operatorname{Lev}_{\alpha}(T): y>x\right\}\right| \geq n$.

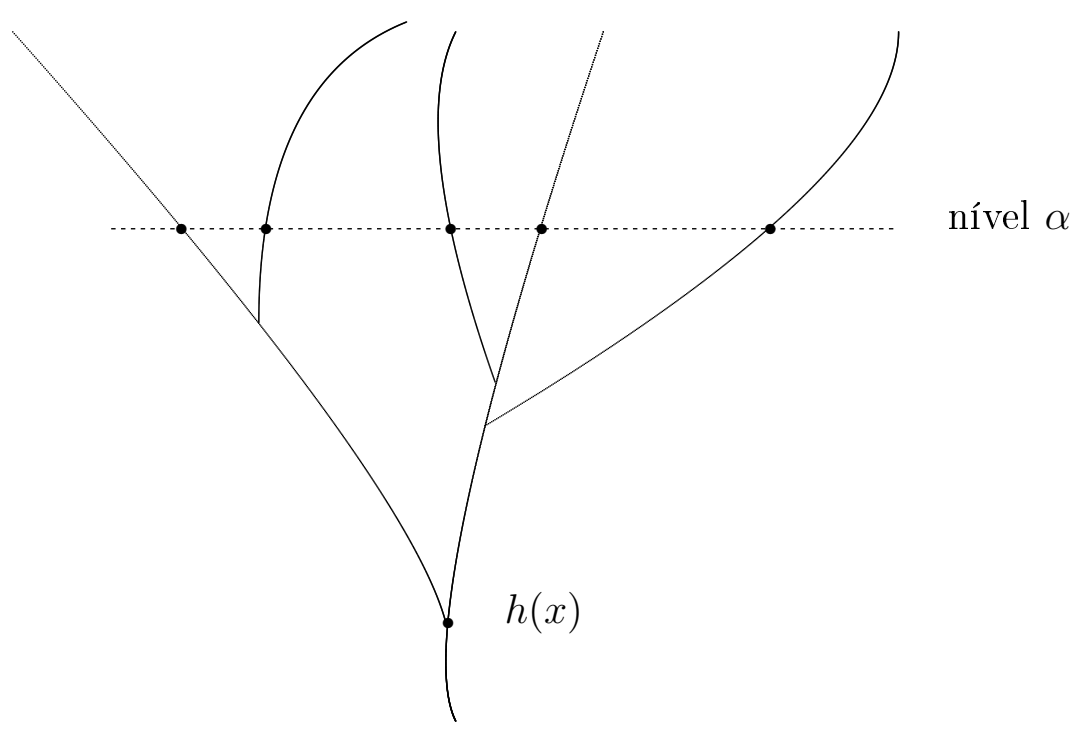

Figura 5.1: Lema 5.5MA implica $S H$ teorema.5.5.

Demonstração. Seja $x \in T$ fixado. Para $n=2$, isso segue do fato que o conjunto $\{y: y>$ $x\}$ "bifurca", pois, caso contrário tal conjunto seria um ramo de tamanho $\omega_{1}$.

Para $n>2$ vamos proceder por indução. Suponha que o lema seja válido para $n=k$. Fixe $\alpha>h(x)$ e $y_{1}, \ldots, y_{k} \in \operatorname{Lev}_{\alpha}(T)$ distintos, onde cada $y_{i}>x$. Agora, seja $\beta>\alpha$, 
tal que existem $z_{k}, z_{k+1} \in \operatorname{Lev}_{\beta}(T)$ distintos, com $z_{k}, z_{k+1}>y_{k}$. Como $T$ é bem podada, temos que existem $z_{i} \in \operatorname{Lev}_{\beta}(T) \operatorname{com} z_{i}>y_{i}$ para $i<k$. Então $\left\{z_{1}, \ldots, z_{k+1}\right\}$ satisfaz o Lema no caso $k+1$.

E agora:

Teorema 5.6. Existe uma árvore de Suslin se, e somente, se existe uma reta de Suslin.

Demonstração. Em primeiro lugar, seja $T$ uma árvore de Suslin. Podemos assumir que $T$ seja bem podada pelo Corolário 1.45Árvoresteorema.1.45.

Agora, seja $L=\{C \subset T: C$ é um ramo de $T\}$. Se $C \in L$, então existe um ordinal $h(C)$ tal que $C$ contém exatamente um elemento de $L e v_{\alpha}(T)$ para todo $\alpha<h(C)$ e para $\alpha \geq h(C)$ temos que $\operatorname{Lev}_{\alpha}(T) \cap C=\emptyset$. Note que, por $T$ ser bem podada $h(C)$ é um ordinal limite.

Para cada $\alpha<h(C)$, seja $C(\alpha)$ o elemento de $C$ no nível $\alpha$.

Vamos definir uma ordem estrita total em $L$.

Fixe uma ordem arbitrária total "ఒ" em $T$ (por exemplo uma boa ordem) ${ }^{10}$. Se $C, D \in L$ e $C \neq D$, seja $d(C, D)$ o menor $\alpha$ tal que $C(\alpha) \neq D(\alpha)$.

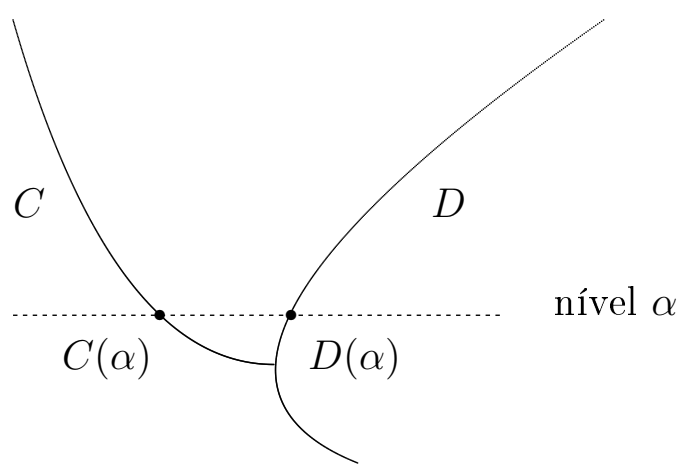

Figura 5.2: Ilustração referente à afirmação anterior.

Observe que $d(C, D)<\min \{h(C), h(D)\}$. Seja $C \triangleleft D$ se, e somente se, $C(d(C, D)) \prec$ $D(d(C, D))$. Afirmamos que " $\triangleleft$ " é uma ordem total.

\footnotetext{
${ }^{10}$ Lembre-se que todo conjunto pode ser bem ordenado.
} 


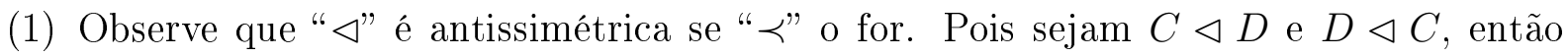
$C(d(C, D) \prec D(d(C, D))$ e $D(d(C, D)) \prec C(d(C, D))$ implicam que $C(d(C, D))=$ $D(d(C, D))$

(2) Sejam $C \triangleleft D$ e $D \triangleleft E$ então $C \triangleleft E$. Veja que $C \triangleleft D$ se, e somente se, $C(d(C, D)) \prec$ $D(d(C, D))$ e $D \triangleleft E \Leftrightarrow D(d(D, E)) \prec E(d(D, E))$. Sejam $d(C, D)=\alpha$ e $d(D, E)=\beta$.

(a) Se $\alpha=\beta$ então $C(\alpha)=C(\beta)$ e $D(\alpha)=D(\beta)$. Note que $d(C, E)=\alpha=\beta$, então $C \triangleleft E$.

(b) Se $\alpha<\beta$ observe que $D(\alpha)=E(\alpha)$ assim $C(\alpha) \prec D(\alpha)=E(\alpha)$. Como $E$ está acima de $D$ segue que $d(C, E)=\alpha$, então $C \triangleleft E$.

(c) Se $\alpha>\beta$ observe que $C(\beta)=D(\beta)$ assim $C(\beta)=D(\beta) \prec E(\beta)$. Como $C$ está acima de $D$ segue que $d(C, E)=\beta$, então $C \triangleleft E$.

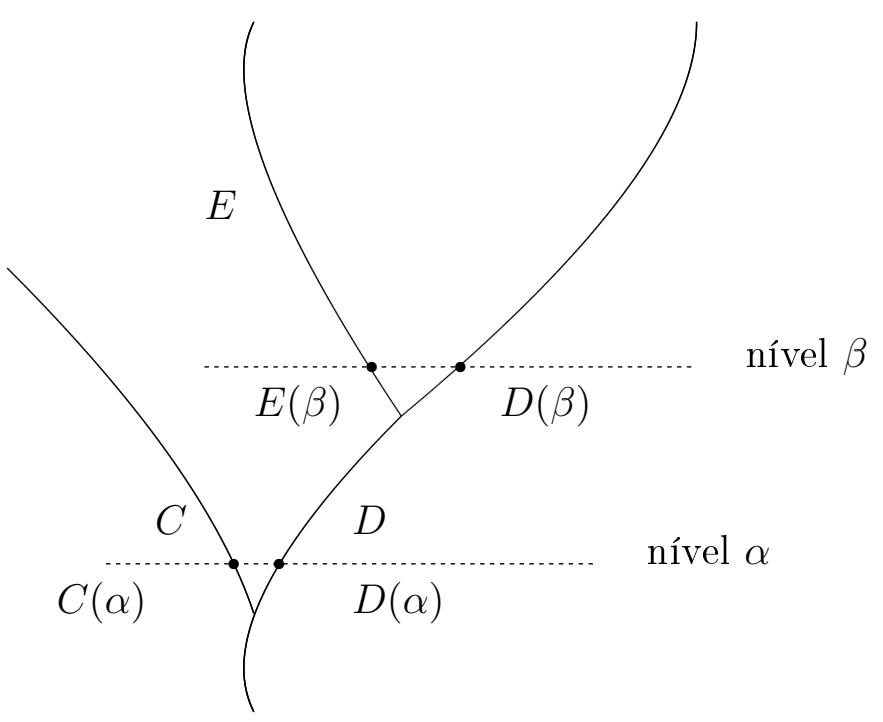

Figura 5.3: Ilustração referente ao caso (b). 


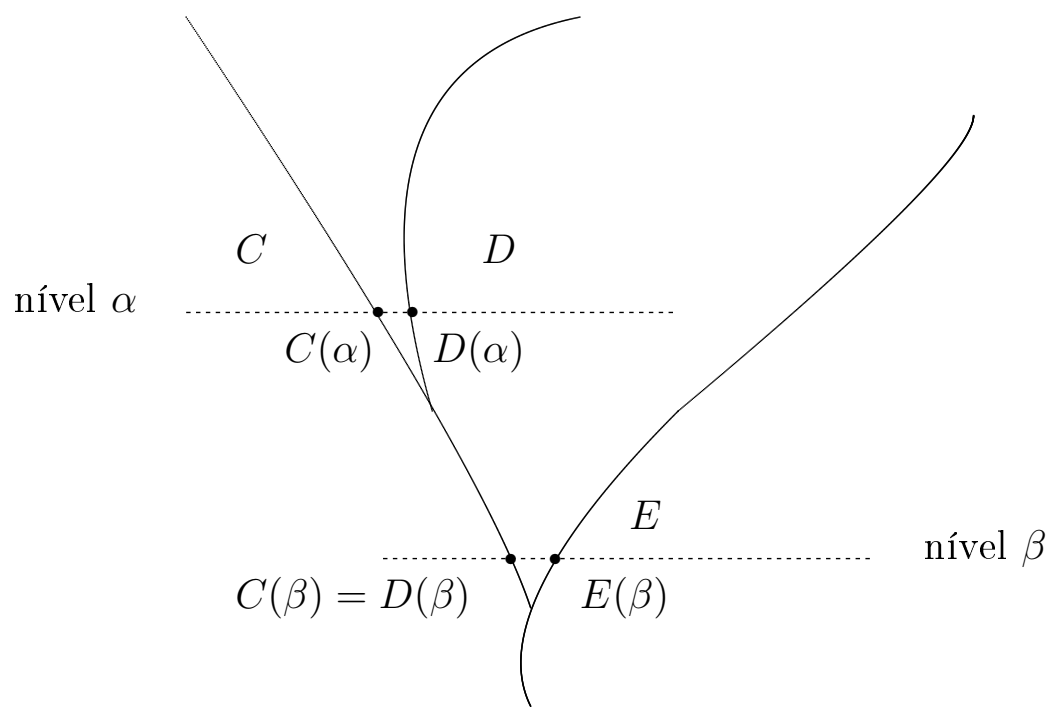

Figura 5.4: Ilustração referente ao caso $(c)$.

(3) “ $\triangleleft$ " é total. Sejam $C, D \in L \operatorname{com} C \neq D$. Então existe $\alpha<\min \{h(C), h(D)\}$ tal que $C(\alpha) \neq D(\alpha)$, pois caso contrário ou $C \varsubsetneqq D$ ou $D \varsubsetneqq C$ e isso contraria a maximalidade das cadeias.

Vamos mostrar $(L, \triangleleft)$ é uma a reta de Suslin.

Para mostrar que $L$ é c.c.c., suponha que $\left\{\left(C_{\xi}, D_{\xi}\right): \xi<\omega_{1}\right\}$ é uma família de intervalos abertos não vazios, dois a dois disjuntos. Tome $E_{\xi} \in\left(C_{\xi}, D_{\xi}\right)$ e tome $\alpha_{\xi}$ tal que $\max \left\{d\left(C_{\xi}, D_{\xi}\right), d\left(E_{\xi}, D_{\xi}\right)\right\}<\alpha_{\xi}<h\left(E_{\xi}\right)$.

Então $\left\{E_{\xi}\left(\alpha_{\xi}\right): \xi<\omega_{1}\right\}$ forma uma anticadeia em $T$. De fato, sejam $E_{\xi}\left(\alpha_{\xi}\right)$ e $E_{\eta}\left(\alpha_{\eta}\right)$ elementos distintos desse último conjunto. Suponha, sem perda de generalidade, que $E_{\xi}\left(\alpha_{\xi}\right) \prec E_{\eta}\left(\alpha_{\eta}\right)$. Então observe que $d\left(C_{\xi}, E_{\xi}\right)=d\left(C_{\xi}, E_{\eta}\right)$ e $\operatorname{assim} C_{\xi}\left(d\left(C_{\xi}, E_{\xi}\right)\right)=$ $C_{\xi}\left(d\left(C_{\xi}, E_{\eta}\right)\right)$ e $E_{\xi}\left(d\left(C_{\xi}, E_{\xi}\right)\right)=E_{\xi}\left(d\left(C_{\xi}, E_{\eta}\right)\right)=E_{\eta}\left(d\left(C_{\xi}, E_{\eta}\right)\right)$, logo $C_{\xi} \triangleleft E_{\eta}$. De maneira análoga, temos $d\left(E_{\xi}, D_{\xi}\right)=d\left(E_{\eta}, D_{\xi}\right)$, então $E_{\xi}\left(d\left(E_{\xi}, D_{\xi}\right)\right)=E_{\xi}\left(d\left(E_{\eta}, D_{\xi}\right)\right)=$ $E_{\eta}\left(d\left(E_{\eta}, D_{\xi}\right)\right)$ e $D_{\xi}\left(d\left(E_{\xi}, D_{\xi}\right)\right)=D_{\xi}\left(d\left(E_{\eta}, D_{\xi}\right)\right)$, logo $E_{\eta} \triangleleft D_{\xi}$. Portanto $] C_{\xi}, D_{\xi}[\cap] C_{\eta}, D_{\eta}[\neq$ 


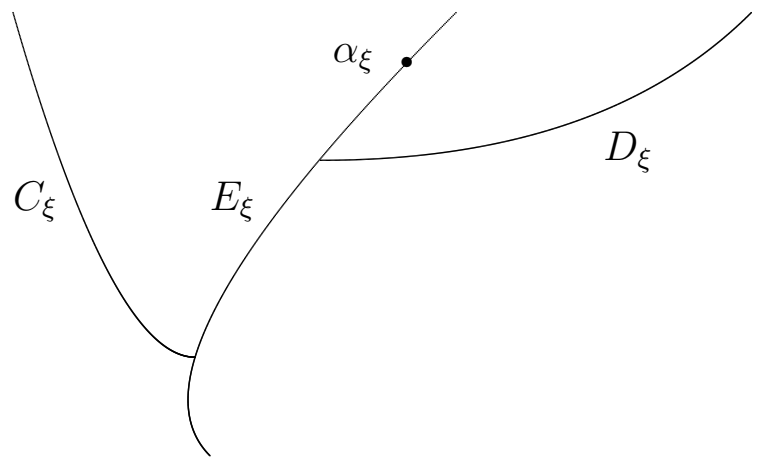

Figura 5.5: Ilustração referente à afirmação anterior.

$\emptyset$. Com isso concluimos que $\left\{E_{\xi}\left(\alpha_{\xi}\right): \xi<\omega_{1}\right\}$ forma uma anticadeia contradizendo o fato de $T$ ser uma árvore de Suslin.

Para mostrar que $L$ não é separável, é suficiente mostrar que para cada $\delta<\omega_{1},\{C \in$ $L: h(C)<\delta\}$ não é denso em $L^{11}$. Fixe $x \in \operatorname{Lev}_{\delta}(T)$. Pelo Lema anterior existe $\alpha>\delta$ com três elementos distintos $y, z, w \in \operatorname{Lev}_{\alpha}(T)$ acima de $x$. Sejam $D, E, F$ elementos de $L$ contendo $y, z, w$ respectivamente. Digamos que possuam a seguinte ordenação $D \triangleleft E \triangleleft F$, então $] D, F$ [ é um intervalo não vazio com $x \in D \cap F$. Porém, $] D, F[$ não contém $C \in L$ tal que $h(C)<\delta$.

Reciprocamente, suponha dada uma reta de Suslin $(L, \triangleleft)$. Pelo Teorema 5.4MA implica $S H$ teorema.5.4 podemos assumir que $L$ é densa sobre si mesma e que nenhum subconjunto aberto não vazio de $L$ é separável.

Seja $\mathcal{J}$ o conjunto de todos os intervalos abertos não vazios de $L$. Então, os elementos de $\mathcal{J}$ são da forma $] a, b[$ onde $a \triangleleft b$.

$\mathcal{J}$ é parcialmente ordenado pela inclusão reversa: $I \leq J$ se, e somente se, $J \subset I$. Vamos definir um subconjunto $T \subset \mathcal{J}$ tal que $(T, \leq)$ é uma árvore de Suslin.

Para construir $T$, primeiro buscaremos $\mathcal{I}_{\beta} \subset \mathcal{J}$ para $\beta<\omega_{1}$ tal que para cada $\beta$ :

(1) Os elementos de $\mathcal{I}_{\beta}$ são dois a dois disjuntos;

(2) $\bigcup_{J \in \mathcal{I}_{\beta}} J$ é denso em $L$;

(3) Se $\alpha<\beta, I \in \mathcal{I}_{\alpha}$ e $J \in \mathcal{I}_{\beta}$ então ou $I \cap J=\emptyset$ ou $(J \subset I$ e $I \backslash \bar{J} \neq \emptyset)$

\footnotetext{
${ }^{11}$ Pois, para cada $A \subset L$ enumerável vai existir um $\zeta<\omega_{1}$, onde $h(C)<\zeta$ para todo $C \in A$.
} 
Assumindo que isso possa ser feito, seja $T=\bigcup_{\beta} \mathcal{I}_{\beta}$. Por $(1)-(3)$, temos:

- $T$ é uma árvore. De fato, seja $I \in T$. Considere $T_{I}=\{J \in T: J \leq I\}$ e seja $A \subset T_{I}$. Seja $H=\bigcup A$, então $H \leq J$ para todo $J \in A$.

- Cada $\mathcal{I}_{\beta}=\operatorname{Lev}_{\beta}(T)$. Por (1) temos que os elementos de $\mathcal{I}_{\beta}$ são dois a dois disjuntos. Temos que, se $I \in \mathcal{I}_{\beta}$, então para cada $\alpha<\beta$ existe um único $J_{\alpha} \in \mathcal{I}_{\alpha}$ tal que $J_{\alpha} \leq I$. De fato, por (3), se $I \in \mathcal{I}_{\beta}$ e $J \in \mathcal{I}_{\alpha} \operatorname{com} \alpha<\beta$ então ou $I \cap J \neq \emptyset$ ou $(I \subset J$ e $J \backslash \bar{I} \neq \emptyset)$. Se para todo $J \in \mathcal{I}_{\alpha}$ implicar que $I \cap J \neq \emptyset$ temos que $\bigcup_{J \in \mathcal{I}_{\alpha}} J$ não é denso em $L$. Logo existe $J_{\alpha} \in \mathcal{I}_{\alpha}$ tal que $I \subset J_{\alpha}$, i.e. $J_{\alpha} \leq I$ para todo $\alpha<\beta$. A unicidade de $J_{\alpha}$ é dada por (1). Portanto $\{J: J \leq I\}$ é equipotênte a $\{\alpha: \alpha<\beta\}$ e $\operatorname{assim} \mathcal{I}_{\beta}=\operatorname{Lev}_{\beta}(T)$.

- Se $A \subset T$ é uma anticadeia, então os elementos de $A$ são dois a dois disjuntos e portanto $|A| \leq \omega$, pois $(L, \triangleleft)$ satisfaz a c.c.c..

- $T$ não possui ramos não enumeráveis. Pois, se $\left\{I_{\xi}: \xi<\omega_{1}\right\}$ fosse um ramo, onde $\xi<\eta$ implica que $I_{\xi}<I \eta$. Logo por $(3)$ se $\xi<\eta$ então $\left(I_{\eta} \subset I_{\xi}\right.$ e $\left.I_{\xi} \backslash \overline{I_{\eta}}\right) \neq \emptyset$. $\operatorname{Assim}\left\{I_{\xi} \backslash \overline{I_{\xi+1}}: \xi<\omega_{1}\right\}$ é uma anticadeia em $(L, \triangleleft)$, o que é uma contradição.

Portanto $T$ é uma árvore de Suslin.

Agora, vamos construir o conjunto $\mathcal{I}_{\beta}$ por indução. Seja $\mathcal{I}_{0}$ uma subfamília maximal disjunta de $\mathcal{J}$, em outras palavras, uma coleção de intervalos abertos dois a dois disjuntos e maximal. A maximalidade de $\mathcal{I}_{0}$ implica que $\bigcup_{I \in \mathcal{I}_{0}} I$ é denso em $T$. Observe que, por vacuidade, vale $(3)$.

Dada $\mathcal{I}_{\alpha}$, definimos $\mathcal{I}_{\alpha+1}$ da seguinte maneira:

Para cada $I \in \mathcal{I}_{\alpha}$, seja $\mathcal{H}_{I}$ uma subfamília maximal disjunta de $\{K \in \mathcal{J}: K \subset I$ e $I \backslash \bar{K} \neq \emptyset\}$. Seja $\mathcal{I}_{\alpha+1}=\bigcup_{I \in \mathcal{I}_{\alpha}} \mathcal{H}_{I}$. Observe que:

(1) Sejam $K_{1}, K_{2} \in \mathcal{I}_{\alpha+1}$ tal que $K_{1} \neq K_{2}$. Se $K_{1}, K_{2} \in \mathcal{H}_{I}$ então $K_{1}, K_{2}$ são disjuntos por definição. Agora, se $K_{1} \in \mathcal{H}_{I}$ e $K_{2} \in \mathcal{H}_{J}$, para $I, J \in \mathcal{I}_{\alpha}$ segue que $K_{1}, K_{2}$ são disjuntos, pois $K_{1} \subset I$ e $K_{2} \subset J \operatorname{com} I \cap J=\emptyset$. 
(2) $\bigcup_{I \in \mathcal{I} \alpha+1} I$ é denso em $L$. Suponha que não seja denso, então existe algum aberto não vazio $A \subset L$ que não intercepta nenhum ponto de $\bigcup_{I \in \mathcal{I} \alpha+1} I$. Como $\bigcup_{I \in \mathcal{I} \alpha} I$ é denso em $L$ implica que existe $J \in \mathcal{I}_{\alpha}$ tal que $J \cap A \neq \emptyset$. Logo $J \cap A$ é um aberto não vazio de $L$. Como nenhum subconjunto aberto de $L$ é separável podemos tomar um subconjunto aberto não vazio $\neq K \subset J \cap A$ tal que $J \cap A \backslash \bar{K} \neq \emptyset$ e então $J \backslash \bar{K} \neq \emptyset$ e portanto $K \in \mathcal{H}_{J}$ contradizendo a maximalidade de $\mathcal{H}_{J}$.

(3) Se $\gamma<\alpha+1, I \in \mathcal{I}_{\gamma}, J \in \mathcal{I}_{\alpha+1}$ ou $I \cap J=\emptyset$ ou $J \subset I$ e $I \backslash \bar{J} \neq \emptyset$. Suponha que $I \cap J \neq \emptyset$ e seja $J^{\prime} \in \mathcal{I}_{\alpha}$ tal que $J \in \mathcal{H}_{J^{\prime}}$ então $I \cap J^{\prime} \neq \emptyset$ o que implica que $J^{\prime} \subset I$ e $I \backslash \overline{J^{\prime}} \neq \emptyset$ e portanto $J \subset J^{\prime} \subset I$ e $I \backslash \bar{J} \neq \emptyset$.

Finalmente, assuma $\gamma$ como um ordinal limite e suponha que tenhamos definido $\mathcal{I}_{\beta}$ para $\beta<\gamma$ satisfazendo (1) $-(3)$. Seja

$$
\begin{gathered}
\mathcal{H}=\left\{K \in \mathcal{J}: \text { para todo } \alpha<\gamma \text { para todo } I \in \mathcal{I}_{\alpha} \text { temos que } I \cap K=\emptyset \text { ou }(K \subset I \text { e }\right. \\
I \backslash \bar{K} \neq \emptyset)\} .
\end{gathered}
$$

Seja $\mathcal{I}_{\gamma}$ uma família maximal de elementos dois a dois disjuntos de $\mathcal{H}$. Note que (1) e (3) são satisfeitas para $\alpha<\beta<\gamma$. Para verificar (2) ( $\operatorname{com} \beta=\gamma$ ) vamos mostrar que para qualquer $J \in \mathcal{J}$ existe $K \in \mathcal{H}$ tal que $K \subset J$. Seja $J \in \mathcal{J}$ e seja $E$ o conjunto de todos os pontos iniciais e finais de todos os intervalos em $\bigcup_{\alpha<\gamma} \mathcal{I}_{\alpha}$. Note que $E$ é enumerável ${ }^{12} \mathrm{e}$, para todo $J \subset L$ aberto não vazio, não é separável. Fixe $K_{1} \in \mathcal{J}$ com $K_{1} \subset J$ e $K_{1} \cap E \neq \emptyset$. Se $I \in \bigcup_{\alpha<\gamma} \mathcal{I}_{\gamma}$, então $K_{1}$ não contém os pontos finais de $I$ (por $J$ não ser separável), logo ou $I \cap K_{1} \neq \emptyset$ ou $K_{1} \subset I$. Agora, tome $K \in \mathcal{J}$ com $K \subset K_{1}$ e $K_{1} \backslash \bar{K} \neq \emptyset$, então $K \subset J$ e $K \in \mathcal{H}^{13}$. Com isso segue (2) da maximalidade de $\mathcal{I}_{\gamma}$.

\subsection{Princípio $\diamond$ implica $\neg S H$}

Finalmente, para terminar a demonstração da independência de $S H$, mostremos que $\diamond$ implica $\neg S H$.

\footnotetext{
${ }^{12}$ Pois $L$ é c.c.c.

${ }^{13} K_{1} \backslash \bar{K} \neq \emptyset \Rightarrow J \backslash \bar{K} \neq \emptyset$.
} 
Definição 5.7. Para qualquer árvore $T$, seja $T_{\alpha}=\bigcup_{\beta<\alpha}\left\{x \in T: x \in \operatorname{Lev}_{\beta}(T)\right\}$. Dizemos que $T_{\alpha}$ é uma sub-árvore de $T$ abaixo de $\alpha$.

Todos os pré requisitos feitos no Capítulo 3Dois Axiomas Extras à ZFCchapter.3 sobre clubs serão úteis nesse próximo lema.

Lema 5.8. Seja $T=\left(\omega_{1}, \triangleleft\right)$ uma árvore. Então:

(a) $\left\{\alpha<\omega_{1}: T_{\alpha}=\alpha\right\}$ é um club em $\omega_{1}$.

(b) Se $A \subset \omega_{1}$ é anticadeia maximal em $T$, então $\left\{\alpha<\omega_{1}: T_{\alpha}=\alpha\right\} \cap\left\{\alpha<\omega_{1}: A \cap T_{\alpha}\right.$ é anticadeia maximal em $\left.T_{\alpha}\right\}$ é um club em $\omega_{1}$.

Demonstração. Para o ítem (a) seja $C=\left\{\alpha<\omega_{1}: T_{\alpha}=\alpha\right\}$. Mostremos que $C$ é fechado. Seja $\gamma \in \bar{C}$, então para todo $\xi<\gamma$ temos que $] \xi, \gamma] \cap C \neq \emptyset$. Para cada $\xi<\gamma$ tome $\left.\left.\eta_{\xi} \in\right] \xi, \gamma\right] \cap C$, então temos que

$$
\gamma=\bigcup_{\eta_{\xi}<\gamma} \eta_{\xi}=\bigcup_{\eta_{\xi}<\gamma} T_{\eta_{\xi}}=\bigcup_{\eta_{\xi}<\gamma} \bigcup_{\beta<\eta_{\xi}}\left\{x \in T: x \in \operatorname{Lev}_{\beta}(T)\right\}
$$

Afirmamos que

$$
\bigcup_{\eta_{\xi}<\gamma} \bigcup_{\beta<\eta_{\xi}}\left\{x \in T: x \in \operatorname{Lev}_{\beta}(T)\right\}=\bigcup_{\beta<\gamma}\left\{x \in T: x \in \operatorname{Lev}_{\beta}(T)\right\} \text {. }
$$

Seja $x \in \bigcup \bigcup\left\{x \in T: x \in \operatorname{Lev}_{\beta}(T)\right\}$ então existe $\eta_{\xi}^{\prime}<\gamma$ tal que $x \in \bigcup_{\beta<\eta_{\xi}^{\prime}}\{x \in T:$ $\left.x \in \operatorname{Lev}_{\beta}(T)\right\}$ então existe $\beta^{\prime}<\eta_{\xi}^{\prime}<\gamma$ tal que $x \in\left\{x \in T: x \in \operatorname{Lev}_{\beta}(T)\right\}$ e assim $x \in \bigcup_{\beta<\gamma}\left\{x \in T: x \in \operatorname{Lev}_{\beta}(T)\right\}$. Para mostrar a outra inclusão, seja $x \in \bigcup_{\beta<\gamma}\{x \in$ $\left.T: x \in \operatorname{Lev}_{\beta}(T)\right\}$. Então existe $\beta^{\prime}<\gamma$ tal que $x \in\left\{x \in T: x \in \operatorname{Lev}_{\beta^{\prime}}(T)\right\}$, ou seja, $x \in \bigcup_{\alpha<\beta^{\prime}}\left\{x \in T: x \in \operatorname{Lev}_{\alpha}(T)\right\} \subset \bigcup_{\beta^{\prime}<\gamma} \bigcup_{\alpha<\beta^{\prime}}\left\{x \in T: x \in \operatorname{Lev}_{\beta^{\prime}}(T)\right\}$. Portanto $\gamma=\bigcup_{\beta<\gamma}\left\{x \in T: x \in \operatorname{Lev}_{\beta}(T)\right\}=T_{\gamma}$.

Portanto $C$ é fechado.

Vamos mostrar que $C$ é ilimitado. Seja $\zeta<\omega_{1}$. Vamos exibir $\beta<\omega_{1}$ tal que $\zeta<\beta$ $\operatorname{com} T_{\beta}=\beta$. Tome $\beta_{0}>\zeta$. Se $\beta_{0}=T_{\beta_{0}}$ acabou. Se não, tome $\beta_{1}<\omega_{1}$ tal que $T_{\zeta}, T_{\beta_{0}} \subset \beta_{1}$ 
14. Tome $\beta_{2}$ tal que $\beta_{1} \subset T_{\beta_{2}}, \beta_{3}$ tal que $T_{\beta_{2}} \subset \beta_{3}$ e assim por diante. Desta forma

$$
T_{\beta_{0}} \subset \beta_{1} \subset T_{\beta_{2}} \subset \beta_{3} \subset T_{\beta_{4}} \subset \cdots
$$

Afirmamos que $\bigcup \beta_{j}=\bigcup T_{\beta_{i}}$, onde $i=0,2,4, \ldots, 2 n, \ldots$ e $j=1,3,5, \ldots, 2 n+1, \ldots$.

Seja $x \in \bigcup \beta_{j}$ então existe $j^{\prime}<\omega$ tal que $x \in \beta_{j^{\prime}} \subset T_{\beta_{j^{\prime}+1}} \subset \bigcup T_{\beta_{i}}$. Para mostrar que $\bigcup T_{\beta_{i}} \subset \bigcup \beta_{j}$, é análogo ao que foi feito acima.

Como a sequência de inclusões é enumerável segue que existe $\beta<\omega_{1}$ tal que $\bigcup \beta_{j}=$ $\beta=\bigcup T_{\beta_{i}}$. Resta saber se $\bigcup T_{\beta_{i}}=T_{\beta}$. Para isso, basta mostrar que $\bigcup T_{\beta_{i}}=T \bigcup \beta_{j}$. Seja $x \in \bigcup T_{\beta_{i}}$ então existe $i^{\prime}<\omega$ tal que $x \in T_{\beta_{i^{\prime}}}$ e $T_{\beta_{i^{\prime}}}=\bigcup_{\alpha<\beta_{i^{\prime}}}\left\{x \in \operatorname{Lev}_{x \in T: \alpha}(T)\right\}$ e $T_{\cup \beta_{j}}=\bigcup_{\alpha<\bigcup \beta_{j}}\left\{x \in T: x \in \operatorname{Lev}_{\alpha}(T)\right\}$, como $\beta_{i^{\prime}}<\bigcup \beta_{j}$ segue que $x \in T_{\beta_{i^{\prime}}} \subset T \cup \beta_{j}$. Agora, seja $x \in T \cup \beta_{j}$ então existe $\alpha^{\prime}<\bigcup \beta_{j}$ tal que $x \in\left\{x \in T: x \in \operatorname{Lev}_{\alpha^{\prime}}(T)\right\} \subset \bigcup T_{\beta_{i}}$, $\operatorname{logo} x \in T_{\bigcup \beta_{j}} \subset T_{\beta_{i^{\prime}}}$

Portanto existe $\beta<\omega_{1} \operatorname{com} \zeta<\beta$ tal que $T_{\beta}=\beta$.

Portanto $C$ é ilimitado.

Para o ítem (b). Se $A \subset \omega_{1}$ é anticadeia maximal em $T$, então seja $D=\left\{\alpha<\omega_{1}\right.$ : $A \cap T_{\alpha}$ é anticadeia maximal em $\left.T_{\alpha}\right\}$.

Veja que $D$ é fechado. De fato, seja $\gamma \in \bar{D}$. Para cada $\xi<\gamma$ temos que $] \xi, \gamma] \cap D \neq \emptyset$. Então para cada $\xi$, tome $\left.\left.\beta_{\xi} \in\right] \xi, \gamma\right] \cap D$. Temos que $A \cap T_{\beta_{\xi}}$ é anticadeia maximal em $T_{\beta_{\xi}}$.

Mostremos que $\bigcup_{\beta_{\xi}<\gamma} A \cap T_{\beta_{\xi}}$ é anticadeia maximal em $\bigcup_{\beta_{\xi}<\gamma} T_{\beta_{\xi}}$. Suponha que existam $x, y \in \bigcup_{\beta_{\xi}<\gamma} A \cap T_{\beta_{\xi}}$ onde, digamos, $x \triangleleft y$. Então $x \in A \cap T_{\beta_{\zeta}}$ e $y \in A \cap T_{\beta_{\eta}}$ para alguns $\left.\left.\beta_{\zeta} \in\right] \zeta, \gamma\right] \cap D$ e $\left.\left.\beta_{\eta} \in\right] \eta, \gamma\right] \cap D$. Como $x \triangleleft y$, segue que $h(x)<h(y)$ e por $T_{\beta_{\eta}}=\bigcup_{\beta<\beta_{\eta}}\left\{x \in T: x \in \operatorname{Lev}_{\beta}(T)\right\}$ implica que $x \in A \cap T_{\beta_{\eta}}$ o que é um absurdo ${ }^{15}$.

Vamos mostrar que $\bigcup_{\beta_{\xi}<\gamma} A \cap T_{\beta_{\xi}}$ é maximal em $\bigcup_{\beta_{\xi}<\gamma} T_{\beta_{\xi}}$. Suponha que não. Então existe $a \in \bigcup_{\beta_{\xi}<\gamma} T_{\beta_{\xi}}$ que não é compatível a todos os elementos de $\bigcup_{\beta_{\xi}<\gamma} A \cap T_{\beta_{\xi}}$ e, além disso, $a \notin \bigcup_{\beta_{\xi}<\gamma} A \cap T_{\beta_{\xi}}$. Mas $a \in T_{\beta_{\xi}}$ para algum $\beta_{\xi}<\gamma$ o que implica que $a \in T_{\beta_{\xi}} \backslash\left(A \cap T_{\beta_{\xi}}\right)$ e isso contradiz a maximalidade $A \cap T_{\beta_{\xi}}$ em $T_{\beta_{\xi}}$. Como $\bigcup_{\beta_{\xi}<\gamma} T_{\beta_{\xi}}=T_{\gamma}$ e $\bigcup_{\beta_{\xi}<\gamma} A \cap T_{\beta_{\xi}}=A \cap\left(\bigcup_{\beta_{\xi}<\gamma} T_{\beta_{\xi}}\right)=A \cap T_{\gamma}$ segue que $A \cap T_{\gamma}$ é anticadeia maximal em $T_{\gamma}$.

\footnotetext{
${ }^{14}$ Isso é possível ser feito, pois $T$ é uma $\omega_{1}$-árvore e como $\zeta, \beta_{0}$ são enumeráveis segue que $T_{\zeta}, T_{\beta_{0}}$ possuem alturas enumeráveis.

${ }^{15}$ Pois $A \cap T_{\beta_{\eta}}$ é anticadeia e $x, y \in A \cap T_{\beta_{\eta}} \operatorname{com} x \triangleleft y$.
} 
Vejamos agora que $D$ é ilimitado. Seja $\zeta<\omega_{1}$ e tome $\beta_{0}>\zeta$. Se $A \cap T_{\beta_{0}}$ for anticadeia maximal em $T_{\beta_{0}}$ acabou. Se não, existe $a \in T_{\beta_{0}} \backslash\left(A \cap T_{\beta_{0}}\right)$ que é incompatível com os elementos de $A \cap T_{\beta_{0}}$. Mas $A$ é anticadeia maximal em $T=\left(\omega_{1}, \triangleleft\right)$. Então existe $b \in A$ tal que $b$ é compatível à $a$. Então, se tomarmos $\left(A \cap T_{\beta_{0}}\right) \cup\{b\}$, conseguimos contornar o problema do elemento " $a$ " que é incompatível a todos os elementos de $A \cap T_{\beta_{0}}$. Observe que $b$ não necessariamente pertence a $T_{\beta_{0}}$ o que implica $h(b)=\beta^{\prime}>\beta$.

Faremos a seguinte construção:

Seja $I_{\beta_{0}}$ o conjunto de todos os pontos que são incompatíveis com $A \cap T_{\beta_{0}}$. Então $I_{\beta_{0}} \cap\left(A \cap T_{\beta_{0}}\right)=\emptyset^{16}$. Seja $A_{\beta_{0}} \subset A$ subconjunto de $A$ dos pontos que são compatíveis aos pontos de $I_{\beta_{0}}$ e seja $\beta_{1}=\sup \left\{h(x): x \in A_{\beta_{0}}\right\}$. Se $\left(A \cap T_{\beta_{0}}\right) \cup A_{\beta_{0}}$ não for anticadeia maximal em $T_{\beta_{1}}$ tome $I_{\beta_{1}}$ o conjunto dos pontos que são incompatíveis com $\left(A \cap T_{\beta_{0}}\right) \cup A_{\beta_{0}}$ e tome $A_{\beta_{1}} \subset A$ subconjunto dos pontos que não são compatíveis com os pontos de $I_{\beta_{1}}$. Seja $\beta_{2}=\sup \left\{h(x): x \in A_{\beta_{0}}\right\}$. Se Se $\left(A \cap T_{\beta_{0}}\right) \cup A_{\beta_{0}} \cup A_{\beta_{1}}$ não for anticadeia maximal em $T_{\beta_{2}}$ prossiga como anteriormente.

Considere $\left(A \cap T_{\beta_{0}}\right) \cup\left(\bigcup_{j<\omega} A_{\beta_{j}}\right)$ e seja $\beta=\sup \left\{h(x): x \in \bigcup_{j<\omega} A_{\beta_{j}}\right\}$ note que $\beta<\omega_{1}$, pois cada $A_{\beta_{j}}$ é no máximo enumerável. Vamos mostrar que $\left(A \cap T_{\beta_{0}}\right) \cup\left(\bigcup_{j<\omega} A_{\beta_{j}}\right)=A \cap T_{\beta}$ e por sua vez é anticadeia maximal em $T_{\beta}$. Claramente $\left(A \cap T_{\beta_{0}}\right) \cup\left(\bigcup_{j<\omega} A_{\beta_{j}}\right) \subset A \cap T_{\beta}$. Seja $a \in A \cap T_{\beta}$. Então $h(a)<\beta$ e assim existe $j^{\prime}$ tal que existe $b \in A_{\beta_{j^{\prime}}} \operatorname{com} h(a)<$ $h(b)<\beta$. Assim,

$$
a \in\left(A \cap T_{\beta_{0}}\right) \cup A_{\beta_{0}} \cup A_{\beta_{1}} \cup \cdots \cup A_{\beta_{j^{\prime}}} \subset\left(\left(A \cap T_{\beta_{0}}\right) \cup \bigcup_{j<\omega} A_{\beta_{j}}\right) .
$$

Pela construção de $A \cap T_{\beta}$ temos a maximalidade em $T_{\beta}$. Portanto $D$ é ilimitado.

Como $C$ e $D$ são $c l u b$ 's segue que $C \cap D$ é $c l u b$ e com isso segue o resultado desse ítem.

A próxima definição e o próximos lemas serão o fio condutor para a construção da árvore de Suslin.

Definição 5.9. Uma árvore $(T, \leq)$ sempre ramifica se, e somente se, para todo $x \in T$ o conjunto $\{y \in T: x<y\}$ não é totalmente ordenado por " $<$ ".

\footnotetext{
${ }^{16}$ Observe que $\left|I_{\beta_{0}}\right|=\omega$.
} 
Lema 5.10. Seja $(T, \leq)$ uma árvore de altura $\omega_{1}$ que sempre ramifica e que toda anticadeia maximal é enumerável, então T é uma árvore de Suslin.

Demonstração. Pelo Lema de Zorn, toda anticadeia está contida em uma anticadeia maximal. Suponha que $B$ seja um ramo não enumerável. Então $B$ intercepta todos os níveis de $T$, caso contrário existiria um nível $\alpha<\omega_{1}$ e um $x \in \operatorname{Lev}_{\alpha}(T)$ tal que $y \leq x$ para todo $y \in B$ e portanto $B$ seria enumerável. Como $T$ sempre ramifica, então, para cada $x \in T$, existe $f(x)>x$ tal que $f(x) \notin B$.

Agora, indutivamente, tome $x_{\alpha} \in B$ para $\alpha<\omega_{1}$ tal que

$$
h\left(x_{\alpha}\right)>\sup \left\{h\left(f\left(x_{\beta}\right)\right): \beta<\alpha\right\} .
$$

Então $\left\{f\left(x_{\alpha}\right): \alpha<\omega_{1}\right\}$ é uma anticadeia não enumerável. De fato, sejam $x_{\alpha}, x_{\eta} \in B$ tal que $x_{\alpha}<x_{\eta}$. Note que não é possível supor que $f\left(x_{\eta}\right)<_{T} f\left(x_{\alpha}\right)$ pois $h\left(f\left(x_{\alpha}\right)\right)<h\left(x_{\eta}\right)<$ $h\left(f\left(x_{\eta}\right)\right)$. Suponha que $f\left(x_{\alpha}\right)<f\left(x_{\eta}\right)$. Então $x_{\eta}<f\left(x_{\eta}\right)$ e $x_{\alpha}<f\left(x_{\alpha}\right)<x_{\eta}<f\left(x_{\eta}\right)$. Observe que se $f\left(x_{\alpha}\right)<x_{\eta}$ então $f\left(x_{\alpha}\right) \in B$ o que é um absurdo. O conjunto $\{t \in T: t<$ $\left.f\left(x_{\eta}\right)\right\}$ é bem ordenado e $x_{\eta}, f\left(x_{\alpha}\right)$ pertencem a esse último. Então $\left\{x_{\eta}, f\left(x_{\alpha}\right)\right\}$ possui um elemento mínimo, ou seja, $f\left(x_{\alpha}\right)<x_{\eta}$ e novamente temos uma cotradição. Portanto $\left\{f\left(x_{\alpha}\right): \alpha<\omega_{1}\right\}$ é uma anticadeia não enumerável.

A partir do resultado anterior todos os nossos esforços serão focados na construção de tal árvore.

Lema 5.11. Seja $T=\left(\omega_{1}, \triangleleft\right)$ uma árvore de altura $\omega_{1}$ que sempre ramifica, $e\left(A_{\alpha}\right)_{\alpha<\omega_{1}}$ uma $\diamond$-sequência. Suponha que para todo ordinal limite $\alpha<\omega_{1}$ se,

$T_{\alpha}=\alpha$ e $A_{\alpha}$ é anticadeia maximal em $\alpha$, implicar que para todo $x \in \operatorname{Lev}_{\alpha}(T)$ existe $y \in A_{\alpha}$ tal que $y \triangleleft x(*)$.

Então T é uma árvore de Suslin.

Demonstração. Pelo Lema 5.10Princípio $\diamond$ implica $\neg S H$ teorema.5.10 é suficiente checar que toda anticadeia maximal, $A \subset \omega_{1}$, é enumerável. 
Como a intersecção de $c l u b$ 's é não vazia e é um $c l u b$ segue do Lema 5.8Princípio $\diamond$ implica $\neg S H$ teorema.5.8 que $C=\left\{\alpha<\omega_{1}: \alpha\right.$ é limite e $T_{\alpha}=\alpha$ e $A \cap T_{\alpha}$ é anticadeia maximal em $\left.T_{\alpha}\right\}$ é um club em $\omega_{1}$.

Usando o fato que $\left(A_{\alpha}\right)_{\alpha<\omega_{1}}$ é uma $\diamond$-sequência temos que $\left\{\alpha: A \cap \alpha=A_{\alpha}\right\}$ é estacionário e daí podemos tomar $\alpha \in C$ tal que $A \cap \alpha=A_{\alpha}$. Note que $A_{\alpha}=A \cap \alpha=A \cap T_{\alpha}$ que é anticadeia maximal em $\alpha=T_{\alpha}$. Por $(*)$, se $z \in T$ e $h(z) \geq \alpha$, então $z$ está acima de algum elemento de $A_{\alpha}=A \cap \alpha$, então $z \notin A^{17}$, pois $A \cap \alpha=A \cap T_{\alpha}$ é anticadeia maximal em $T_{\alpha}=\alpha$.

Portanto $A=A_{\alpha}$, como $A_{\alpha} \subset \omega_{1}$ temos que $A$ é enumerável.

Enfim, o teorema desejado.

Teorema 5.12. $\diamond$ implica que existe uma árvore de Suslin.

Demonstração. Seja $I_{\beta}=\{\omega \cdot \beta+n: n \in \omega\}$. Fixe uma $\diamond$-sequência $\left(A_{\alpha}\right)_{\alpha<\omega_{1}}$. A árvore $T$ será $\left(\omega_{1}, \triangleleft\right)$ onde a ordem “ $\triangleleft$ ” será indutivamente construída de forma que:

(1) $\triangleleft$ é uma ordem que induz uma árvore em $\omega_{1}$ e para cada $\beta<\omega_{1}$ temos $\operatorname{Lev}_{\beta}(T)=I_{\beta}$.

(2) Para cada $\beta<\omega_{1}$ e $n<\omega,(\omega \cdot \beta+n) \triangleleft(\omega \cdot(\beta+1)+2 n)$ e $(\omega \cdot \beta+n) \triangleleft(\omega \cdot(\beta+1)+2 n+1)$.

(3) Se $\beta<\alpha<\omega_{1}$ e $x \in I_{\beta}$, então existe $y \in I_{\alpha}$ tal que $x \triangleleft y$.

(4) vale $(*)$ do Lema 5.11Princípio $\diamond$ implica $\neg S H$ teorema.5.11.

Assumindo que $\triangleleft$ possa ser construido, (1) e (2) garantem que $T$ é uma árvore de altura $\omega_{1}$ que sempre ramifica e (4) implica que $T$ é de Suslin. A condição (3) irá facilitar a construção de $T$. Note agora que $T_{\alpha}=\omega \cdot \alpha$ por $(1)^{18}$.

Para construir $\triangleleft$ indutivamente, vamos assumir que $\triangleleft$ está definido para os elementos de $\omega \cdot \alpha$ tal que (1)-(4) valem abaixo de $\alpha$ e vamos descrever como estender $\triangleleft$ para elementos de $\omega \cdot \alpha \cup I_{\alpha}$.

\footnotetext{
${ }^{17}$ Pois caso contrário $z$ seria compatível com algum elemento de $A_{\alpha} \subset A$.

${ }^{18} T_{\alpha}=\bigcup_{\gamma<\alpha}\left\{x \in T: x \in \operatorname{Lev}_{\gamma}(T)\right\}=\bigcup_{\gamma<\alpha}\left\{x \in T: x \in I_{\gamma}\right\}=\bigcup_{\gamma<\alpha} I_{\gamma}=\bigcup_{\gamma<\alpha}\{\omega \cdot \beta+n: n+\omega\}=$ $\omega \cdot \alpha$.
} 
Se $\alpha=\beta+1$, então a condição (2) especifica a construção: Se $x \in \omega \cdot \alpha$, então $x \triangleleft(\omega \cdot \alpha+2 n)$ se, e somente se, $x=(\omega \cdot \beta+n)$ ou $x \triangleleft(\omega \cdot \beta+n)$; da mesma forma que $x \triangleleft(\omega \cdot \alpha+2 n+1)$. Isso preserva (1),(3) e (4) nada diz sobre sucessores de $\alpha$.

Agora, assuma que $\alpha$ é um ordinal limite. Para cada $x \in T_{\alpha}=\omega \cdot \alpha$, seja $B(x)$ uma cadeia em $T_{\alpha}$, tal que $x \in B(x)$ e $B(x)$ intercepta $I_{\eta}=\operatorname{Lev}_{\eta}\left(T_{\alpha}\right)$ para cada $\eta<\alpha$.

Para encontrar tal $B(x)$, primeiro escolha $\xi_{m}=\xi_{m}(x)$ para $m<\omega$ tal que $h(x)<\xi_{0}<$ $\xi_{1}<\cdots$ e $\sup \left\{\xi_{m}: m \in \omega\right\}=\alpha$, note que isso é possível pelo fato de $\alpha$ ser enumerável. Indutivamente escolha $y_{m}=y_{m}(x) \in I_{\xi_{m}}$ tal que $x \triangleleft y_{0} \triangleleft y_{1} \triangleleft \cdots$ e observe que isso é possível por (3). Defina o conjunto

$$
B(x)=\left\{z \in T_{\alpha} \text { : existe } n \text { tal que } z \triangleleft y_{n}(x)\right\} .
$$

Agora, seja $\omega \cdot \alpha=\left\{x_{n}: n \in \omega\right\}$, e defina, para cada $z \in \omega \cdot \alpha$ temos $z \triangleleft(\omega \cdot \alpha+n)$ se, e somente se, $z \in B\left(x_{n}\right)$. Pelo fato de $B\left(x_{n}\right)$ interceptar cada nível de $T_{\alpha}$ implica que $\omega \cdot \alpha+n$ de fato possui altura $\alpha$ em $T$.

Finalmente, a condição (4) no nível $\alpha$ é um problema somente se $\omega \cdot \alpha=\alpha$ e $A_{\alpha}$ é anticadeia maximal em $T_{\alpha}$. Então, vamos supor esse caso. Modificando a construção de $B(x)$ para $x \in T_{\alpha}$ por, em primeiro lugar escolhendo $y_{0}(x)$ tal que $x \triangleleft y_{0}(x)$ e existe $z \in A_{\alpha}$ tal que $z \triangleleft y_{0}(x)$ (isso é possível pois $x$ é comparável com algum elemento de $A_{\alpha}$, caso contrário $A_{\alpha}$ não seria maximal em $T_{\alpha}$. $)$ Então $\xi_{0}(x)=h\left(y_{0}(x)\right)$. Agora escolha $\xi_{m}(x)$ para $1<m \leq \omega$ e $y_{m}(x)$ para $1<m \leq \omega$ como antes. Então cada $B(x)$ intercepta $A_{\alpha}$, com isso vale $(*)$. 


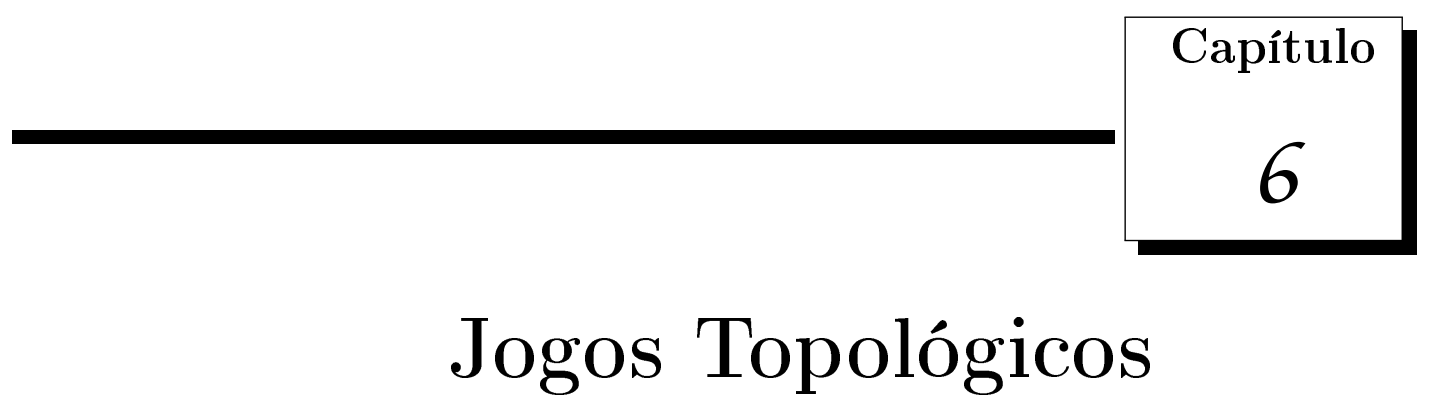

Intuitivamente um jogos topológico é uma maneira de "enxergar" um espaço (ou propriedade topológica) de um jeito mais simples. Por meio de jogadores (nos jogos que falaremos serão dois, jogadores $I$ e $I I)$ que executam suas jogadas, estas podendo ser conjuntos, pontos, conjuntos abertos, coberturas abertas, etc. É dito em quantas jogadas serão necessárias para encerrar o jogo, podendo ser em uma quantidade finita, enumerável ou também não-enumerável. E é dito também o critério sobre quem é o vencedor.

O termo jogo topológico foi introduzido pela primeira vez por Berge em 1957 e em 1965 seu formalismo foi feito por Pears. Rastislav Telgársky em 1975 e 1977 definiu o conceito de "propriedades topológicas definidas por jogos" e mais tarde "espaços definidos por jogos topológicos". Depois de mais de 35 anos, o termo "jogo topológico" generalizou-se e apareceram centenas de publicações. Como veremos na seção seguinte, a idéia de jogos é anterior ao formalismo de Berge. Para mais detalhes sobre a história dos jogos topológicos veja [13].

\subsection{Jogo de Choquet (Banach Mazur)}

O jogo de Banach Mazur apareceu pela primeira vez no famoso Livro Escocês (Scottish Book). Esse livro tem uma história interessante, foi criado entre 1935-1941 na cidade de Lwów que naquele tempo fazia parte da Polônia. Um grupo de pessoas que trabalhavam 
na Universidadede Lwów, entre eles nomes que são bem conhecidos como S. Banach, S. Mazur, S. Ulam, H. Steinhaus e outros. Esse grupo frequentemente se reunia para discutir informalmente problemas de matemática no The Scottish Caffé House (daí o nome do livro). Seguindo a idéia de Banach, um caderno foi comprado e deixado aos cuidados do garçom do estabelecimento. Cada problema proposto (ou solução) era guardada nesse caderno. Dizem que o prêmio para as soluções dos problemas propostos era uma garrafa de vinho ou uma de cerveja (será que dependia da dificuldade do problema?).

O Problema 43 do livro escocês foi proposto por Mazur: Sejam dois jogadores (Mazur denotou por $A$ e $B$ ) e seja $E$ um subconjunto de $\mathbb{R}$. O jogo é definido como: $A$ toma um intervalo não vazio $D_{1}$, então $B$ toma um sub-intervalo não vazio $D_{2}$ de $D_{1}$. $A$ continua com $D_{3}$ sub-intervalo de $D_{2}$ e assim por diante. $A$ vence se a intersecção de todos intervalos $D_{1}, D_{2}, \ldots$ possui um ponto em comum com $E$. Caso contrário $B$ vence. Mazur tinha observado o seguinte: se o complementar de $E$ é de $1^{\text {a }}$ categoria em algum intervalo, então o jogador $A$ possui uma regra que dará a sua vitória nesse jogo. Agora, se $E$ for de $1^{\text {a }}$ categoria em $\mathbb{R}$ então o jogador $B$ possuirá uma regra que lhe dará a vitória nesse jogo. A questão proposta por Mazur (que valia uma garrafa de vinho!) era se essas duas condições eram necessárias para a vitória dos jogadores $A$ e $B$ respectivamente. A resposta foi dada em 4 de agosto de 1935 por Banach e era verdadeira. Porém a prova de Banach nunca foi publicada.

Muito foi feito a partir do que foi proposto por Mazur. Veremos um caso particular proposto por Choquet.

Definição 6.1. Seja $X$ um espaço topológico não vazio. O jogo de Choquet $G_{X}$ de $X$ é definido como: Os jogadores I e II escolhem em cada turno abertos não vazios de $X$

$\begin{array}{ccccc}I & U_{0} & & U_{1} & \\ I I & & V_{0} & & V_{1}\end{array}$

tais que $U_{0} \supseteq V_{0} \supseteq U_{1} \supseteq V_{1} \supseteq \ldots$. Dizemos que II vence o jogo se $\bigcap_{n \in \omega} V_{n}\left(=\bigcap_{n \in \omega} U_{n}\right) \neq \emptyset$. Portanto I vence o jogo se $\bigcap_{n \in \omega} V_{n}\left(=\bigcap_{n \in \omega} U_{n}\right)=\emptyset$.

Uma estratégia para I nesse jogo é uma "regra" que diz como ele deve jogar, para cada $n \in \omega$, no seu $n$-ésimo movimento $U_{n}$, dadas as jogadas $V_{0}, \ldots, V_{n-1}$ do jogador $I I$ e as 
jogadas dele próprio $U_{0}, \ldots, U_{n-1}$.

Formalmente, isso é definido como: Seja $T$ a árvore das posições legais em $G_{X}$, i.e., $T$ consiste de todas as sequências finitas $\left(W_{0}, \ldots, W_{n}\right)$, onde $W_{i}$ é um subconjunto aberto não vazio de $X$ e $W_{0} \supseteq W_{1} \supseteq \cdots \supseteq W_{n}$ (observe que $(T, \leq$, onde $\leq$ é $\supseteqq$ ). Portanto $T$ é uma árvore bem podada em $\{W \subset X: W$ é aberto não vazio de $X\}$.

Uma estratégia para $I$ em $G_{X}$ é uma sub-árvore $\sigma \subset T$ tal que:

(i) $\sigma$ é não vazio.

(ii) se $\left(U_{0}, V_{0}, \ldots, U_{n}\right) \in \sigma$ então para todo aberto não vazio $V_{n} \subset U_{n}$, $\left(U_{0}, V_{0} \ldots, U_{n}, V_{n}\right) \in \sigma$.

(iii) se $\left(U_{0}, V_{0}, \ldots, U_{n-1}, V_{n-1}\right) \in \sigma$, então existe um único $U_{n}$ tal que $\left(U_{0}, V_{0}, \ldots, U_{n-1}, V_{n-1}, U_{n}\right) \in \sigma$.

Intuitivamente, a estratégia $\sigma$ funciona da seguinte maneira: $I$ começa jogando $U_{0}$ onde $\left(U_{0}\right) \in \sigma$ (e é único por $(i i i)$ ), II então joga qualquer aberto não vazio $V_{0} \subset U_{0}$; por (ii), $\left(U_{0}, V_{0}\right) \in \sigma$. Então $I$ responde jogando o único aberto não vazio $U_{1} \subset V_{0}$ tal que $\left(U_{0}, V_{0}, U_{1}\right) \in \sigma$.

Este próximo resultado mostra que o Jogo de Choquet está relacionado com a Propriedade de Baire.

Teorema 6.2 (Oxtoby). Um espaço topológico não vazio $X$ é de Baire se, e somente se, o jogador I não possui estratégia vencedora em $G_{X}$.

Demonstração. Suponha que $X$ não seja de Baire e vamos mostrar que $I$ tem estratégia vencedora. Então existe $U_{0} \subset X$ aberto não vazio e $\left(G_{n}\right)_{n \in \omega}$ uma família de abertos densos em $X \operatorname{com} U_{0} \cap\left(\bigcap_{n \in \omega} G_{n}\right)=\emptyset$. O jogador $I$ começa jogando tal $U_{0}$. Se $I I$ joga $V_{0} \subset U_{0}$, temos que $V_{0} \cap G_{0} \neq \emptyset$, então $I$ pode jogar $U_{1}=V_{0} \cap G_{0} \subset V_{0}$. Logo $I I$ joga $V_{1} \subset U_{1}$ e $I$ joga $U_{2}=V_{1} \cap G_{1} \subset V_{1}$ e assim por diante. Temos $\bigcap_{n \in \omega} U_{n} \subseteq \bigcap_{n \in \omega}\left(G_{n} \cap U_{0}\right)=$ $\left(\bigcap_{n \in \omega} G_{n}\right) \cap U_{0}=\emptyset$. Com isso descrevemos uma estratégia vencedora para $I$.

Suponha que $I$ possui uma estratégia vencedora $\sigma$ e vamos mostrar que $X$ não é de Baire.. Seja $U_{0}$ o primeiro movimento de acordo com $\sigma$. Mostremos que $U_{0}$ não é de 
Baire. Para isso, construiremos uma sub-árvore não vazia bem podada $S \subset \sigma$ tal que para qualquer $p=\left(U_{0}, V_{0}, \ldots, U_{n}\right) \in S$ o conjunto $\mathcal{U}_{p}=\left\{U_{n+1}:\left(U_{0}, V_{0}, \ldots, U_{n}, V_{n}, U_{n+1}\right) \in S\right\}$ consiste de abertos dois a dois disjuntos e $\bigcup \mathcal{U}_{p}$ denso em $U_{n}$. Assumindo a construção, seja $W_{n}=\bigcup\left\{U_{n}:\left(U_{0}, V_{0}, \ldots, U_{n}\right) \in S\right\}$. Segue que $W_{n}$ é aberto e denso em $U_{0}$ para cada $n<\omega^{1}$. Afirmamos que $\bigcap_{n \in \omega} W_{n}=\emptyset$. Caso contrário, se $x \in \bigcap_{n \in \omega} W_{n}$, existe um único $\left(U_{0}, V_{0}, U_{1}, V_{1} \ldots\right) \in[S] \operatorname{com} x \in U_{n}$ para cada $n$. Então $\bigcap_{n \in \omega} U_{n} \neq \emptyset$, contradizendo o fato de que $\left(U_{0}, V_{0}, U_{1}, V_{1} \ldots\right) \in[\sigma]$ e $\sigma$ ser a estratégia vencedora de $I$. Assim, $U_{0} \cap\left(\bigcap_{n \in \omega} W_{n}\right)=$ $\emptyset$. Portanto $U_{0}$ não é de Baire o que implica que $X$ não é de Baire.

Para construir $S$ determinamos indutivamente sequências de $\sigma$ de tamanho $n$ e incluimos em $S$. Se $\left(U_{0}, V_{0}, \ldots, U_{n-1}, V_{n-1}\right) \in S$, então $\left(U_{0}, V_{0}, \ldots, U_{n-1}, V_{n-1}, U_{n}\right) \in S$ para um único $U_{n} \operatorname{com}\left(U_{0}, V_{0}, \ldots, U_{n-1}, V_{n-1}, U_{n}\right) \in \sigma^{2}$. Agora, se $p=\left(U_{0}, V_{0}, \ldots, U_{n}\right) \in S$ note que para qualquer aberto não vazio $V_{n} \subset U_{n}$ se $V_{n}^{*}=U_{n+1}$ é a jogada que $\sigma$ pede que o jogador $I$ faça em seguida, então $U_{n+1}$ é um subconjunto aberto não vazio de $V_{n}$. Usando o Lema de Zorn (ou o argumento de exaustão transfinita) seja $\mathcal{V}_{p}$ a coleção maximal de subconjuntos abertos nãos vazios $V_{n} \subset U_{n}$ tal que $\left\{V_{n}^{*}: V_{n} \in \mathcal{V}_{p}\right\}$ são dois a dois disjuntos. Acrescente em $S$ todos $\left(U_{0}, V_{0}, \ldots, U_{n}, V_{n}, V_{n}^{*}\right) \operatorname{com} V_{n} \in \mathcal{V}_{p}$. Então $\mathcal{U}_{p}=\left\{U_{n+1}:\left(U_{0}, V_{0}, \ldots, U_{n}, V_{n}, U_{n+1}\right) \in S\right\}=\left\{V_{n}^{*}: V_{n} \in \mathcal{V}_{p}\right\}$ é uma família maximal em que seus elementos são dois a dois disjuntos e $\bigcup\left\{U_{n+1} \in \mathcal{U}_{p}\right\}$ é denso em $U_{n}$ pela maximalidade de $\mathcal{V}_{p}$, pois se $\tilde{V}_{n} \subset U_{n}$ é um aberto não vazio disjunto de $\bigcup\left\{U_{n+1} \in \mathcal{U}_{p}\right\}$, então $\mathcal{V}_{p} \cup\left\{\tilde{V}_{n}\right\}$ viola a maximalidade de $\mathcal{V}_{p}$.

Definição 6.3. Um espaço topológico não vazio é dito ser um espaço de Choquet se o jogador II possui estratégia vencedora no jogo de Choquet.

Proposição 6.4. O produto de dois espaços de Choquet é um espaço de Choquet.

Demonstração. Sejam $X$ e $Y$ espaços topológicos de Choquet, i.e., o jogador $I I$ possui estratégia vencedora em $G_{X}$ e em $G_{Y}$. Agora considere $G_{X \times Y}$. Sejam $I_{X \times Y}, I I_{X \times Y}$ jogadores de $G_{X \times Y} ; I_{X}, I I_{X}$ jogadores de $G_{X} ; I_{Y}, I I_{Y}$ jogadores de $G_{Y}$.

\footnotetext{
${ }^{1}$ segue de $\bigcup \mathcal{U}_{p}$ ser denso.

${ }^{2}$ isso se dá pelo ítem $($ iii $)$
} 
Seja $U_{0}$ a primeira jogada de $I_{X \times Y}$, então podemos tomar $U_{0}^{X} \times U_{0}^{Y} \subset U_{0}$ onde $U_{0}^{X} \subset X$ e $U_{0}^{Y} \subset Y$ são abertos não vazios. Usando as respectivas estratégias vencedoras de $I I_{X}$ em $G_{X}$ e $I I_{Y}$ em $G_{Y}, I I_{X}$ responde com $V_{0}^{X} \subset U_{0}^{X}$ e $I I_{Y}$ responde com $V_{0}^{Y} \subset U_{0}^{Y}$. Então $I I_{X \times Y}$ responde com $V_{0}:=V_{0}^{X} \times V_{0}^{Y} \subset U_{0}^{X} \times U_{0}^{Y} \subset U_{0}$ no jogo $G_{X \times Y}$. Agora $I_{X \times Y}$ joga com $U_{1} \subset V_{0}$. Novamente, podemos tomar $U_{1}^{X} \times U_{1}^{Y} \subset U_{1}$ e assim $I I_{X}$ responde com $V_{1}^{X} \subset U_{1}^{X}$ e $I I_{Y}$ responde com $V_{1}^{Y} \subset U_{1}^{Y}$. Logo, $I I_{X \times Y}$ responde com $V_{1}:=V_{1}^{X} \times V_{1}^{Y} \subset U_{1}^{X} \times U_{1}^{Y} \subset U_{1}$.

Continuando esse processo recursivavente teremos:

$$
U_{0} \supset V_{0} \supset U_{1} \supset V_{1} \supset \cdots U_{n} \supset V_{n} \supset \cdots
$$

$\operatorname{com} \bigcap_{n \in \omega} U_{n}=\bigcap_{n \in \omega} V_{n}=\bigcap_{n \in \omega}\left(V_{n}^{X} \times V_{n}^{Y}\right)=\left(\bigcap_{n \in \omega} V_{n}^{X} \times \bigcap_{n \in \omega} V_{n}^{Y}\right) . \operatorname{Como} \bigcap_{n \in \omega} V_{n}^{X} \neq \emptyset$ e $\bigcap_{n \in \omega} V_{n}^{Y} \neq \emptyset$ temos que $\bigcap_{n \in \omega} U_{n}=\bigcap_{n \in \omega} V_{n} \neq \emptyset$.

Assim, podemos mostrar qual a relação entre as propriedades de Choquet e de Baire.

Corolário 6.5. Se X é um espaço de Choquet então X é de Baire. Porém, a recíproca não é verdadeira.

Demonstração. O espaço $X$ é de Choquet se o jogador $I I$ tem estatégia vencedora no jogo de Choquet. Isso implica que o jogador $I$ não possui estratégia vencedora. Então pelo Teorema de Oxtoby segue que $X$ é de Baire.

Agora, provemos que a recíproca não é verdadeira. Suponha, por absurdo, que se $X$ é de Baire implica que $X$ seja de Choquet. Então $X \times X$ é de Choquet pela Proposição anterior. Daí, pelo parágrafo anterior, temos que $X \times X$ é de Baire. Logo isso implicaria que o quadrado de espaços de Baire é de Baire. Mas em [3] existe um exemplo de um espaço de Baire cujo o quadrado não é de Baire.

Mesmo não valendo que todo espaço de Baire é de Choquet, ainda temos, por exemplo: Proposição 6.6. Seja X um espaço topológico compacto Hausdorff. Então X é de Choquet. 
Demonstração. Note que por $X$ ser compacto e Hausdorff implica que $X$ é regular. Seja $U_{0} \subset X$ a primeira jogada de $I$. Tome $x_{0} \in U_{0}$. Então existe $V_{0} \subset U_{0}$ aberto em $X$ tal que $x_{0} \in V_{0} \subset \overline{V_{0}} \subset U_{0}$. Então $I I$ responde com $V_{0} \subset U_{0}$. Na $n$-ésima jogada, tome $x_{n-1} \in U_{n-1}$ e seja $V_{n-1} \subset U_{n-1}$ tal que $x_{n-1} \in V_{n-1} \subset \overline{V_{n-1}} \subset U_{n-1}$. Assim $\left\{V_{n}\right\}_{n<\omega}$ são todas as jogadas de $I I \operatorname{com} V_{n} \subset \overline{V_{n}} \subset U_{n} \subset V_{n-1} \subset \overline{V_{n-1}}$ para todo $n<\omega$. Daí $\bigcap_{n<\omega} V_{n}=\bigcap_{n<\omega} \overline{V_{n}}$. Como $\left\{\overline{V_{n}}\right\}_{n<\omega}$ tem p.i.f. segue do Teorema 2.17Noções Básicas de Topologiateorema.2.17 que $\bigcap_{n<\omega} V_{n}=\bigcap_{n<\omega} \overline{V_{n}} \neq \emptyset$. Portanto o jogador $I I$ tem estratégia vencedora.

Corolário 6.7. Espaços localmente compactos Hausorff são de Choquet.

Demonstração. SEja $X$ um espaço localmente compacto Hausdorff. Seja $U_{0} \subset X$ a primeira jogada de $I$. Seja $x^{\prime} \in U_{0}$ e $C \subset U_{0}$ uma vizinhança compacta de $x^{\prime}$. Então $I I$ responde com $V_{0}=V_{0}^{\prime} \cap C$ onde $V_{0}^{\prime} \subset U_{0}$ é aberto não vazio. Daí continua com os mesmos passos da proposição anterior.

\subsection{Jogo de Rothberger}

O jogo de Rothberger deriva-se da Propriedade de Rothberg, enunciada pelo mesmo em 1938. Um espaço topológico $X$ é dito ter a propriedade de Rothberger se para toda sequência $\left(\mathcal{U}_{n}\right)_{n \in \omega}$ de coberturas abertas de $X$, existe uma sequência $\left(U_{n}\right)_{n \in \omega}$ tal que para cada $n \in \omega, U_{n} \in \mathcal{U}_{n}$, e $\bigcup_{n \in \omega} U_{n}=X$.

Definição 6.8 (jogo de Rothberger). Seja X um espaço topológico não. Sejam I e II jogadores que executam as seguintes jogadas:

I: Escolhe $\mathscr{C}_{0}$ cobertura de abertos para $X$.

II: Toma $C_{0} \in \mathscr{C}_{0}$

I: Escolhe $\mathscr{C}_{1}$ cobertura de abertos para $X$.

II: Toma $C_{1} \in \mathscr{C}_{1}$ 
I: Escolhe $\mathscr{C}_{n}$ cobertura de abertos para $X$.

II: Toma $C_{n} \in \mathscr{C}_{n}$

O jogador II vence se $\left\{C_{n}: n \in \omega\right\}$ é uma cobertura para $X$.

Definição 6.9. Dizemos que X é um espaço de Rothberger se o jogador I não tem uma estratégia vencedora para o jogo de Rothberger.

Observação 6.10. X é um espaço de Rothberger se, e somente se o jogador I não tem uma estratégia vencedora para o jogo de Rothberger.

Se $X$ não é Lindelöf implica que $I$ possui estratégia vencedora no jogo de Rothberger. De fato, seja $\mathscr{C}$ uma cobertura aberta não enumerável para $X$ que $\mathscr{C}$ não possua subcobertura enumerável. Logo, o jogador $I$ pode jogar com a mesma cobertura $\mathscr{C}$ em todas as jogadas. Como a quantidade de escolhas que o jogador II pode fazer é enumerável temos que $\left\{C_{n}: n \in \omega\right\}$ não cobre $X$ e portanto $I$ vence. Note que com isso temos que Rothberger implica Lindelöf ${ }^{3}$.

Se $X$ é enumerável, temos que $I I$ possui estratégia vencedora. De fato, seja $X=\left\{x_{n}: n \in \omega\right\}$ uma enumeração dos elementos de $X$. Sejam as seguintes jogadas:

I: Escolhe $\mathscr{C}_{0}$ cobertura de abertos para $X$.

$I I:$ Toma $C_{0} \in \mathscr{C}_{0}$ tal que $C_{0} \ni x_{0}$

I: Escolhe $\mathscr{C}_{1}$ cobertura de abertos para $X$.

$I I:$ Toma $C_{1} \in \mathscr{C}_{1}$ tal que $C_{1} \ni x_{1}$

I: Escolhe $\mathscr{C}_{n}$ cobertura de abertos para $X$.

$I I:$ Toma $C_{n} \in \mathscr{C}_{n}$ tal que $C_{n} \ni x_{n}$

\footnotetext{
${ }^{3} X$ é de Rothberger se $I$ não possui estratégia vencedora $\Rightarrow X$ Lindelöf.
} 
Logo $\left\{C_{n}: n \in \omega\right\}$ cobre $X$. Portanto $I I$ tem estratégia vencedora no jogo de Rothberger.

Apesar de todo espaço de Rothberger ser um espaço de Lindelöf, nem todo compacto é de Rothberger como veremos a seguir.

Proposição 6.11. $2^{\omega}$ é compacto e o jogador I possui estratégia vencedora no jogo de Rothberger.

Demonstração. Como cada $2=\{0,1\}$ é compacto segue pelo Teorema de Tychonoff que $2^{\omega}$ é compacto. Observe que $2^{\omega}=\{f: \omega \rightarrow 2\}$ e seja $F_{n, k}=\{f: \omega \rightarrow 2 \mid f(n)=k\}$

${ }^{4}$. Considere as seguintes jogadas feitas por $I$ :

$$
\begin{gathered}
\mathscr{C}_{0}=\left\{F_{0,0}, F_{0,1}\right\}, \\
\mathscr{C}_{1}=\left\{F_{1,0}, F_{1,1}\right\}, \\
\mathscr{C}_{2}=\left\{F_{2,0}, F_{2,1}\right\}, \\
\vdots \\
\mathscr{C}_{n}=\left\{F_{n, 0}, F_{n, 1}\right\},
\end{gathered}
$$

Suponha que o jogador $I I$ tenha feito as seguintes jogadas $\left\{F_{0, j_{0}}, F_{1, j_{1}}, \ldots, F_{n, j_{n}}, \ldots\right\}:=$ $\mathscr{C}$ onde $j_{n} \in\{0,1\}$. Se $j_{n}=0$ defina $j_{n}^{\prime}=1$ e vice e versa. Seja $f: \omega \rightarrow 2$ dada por $f(n)=j_{n}^{\prime}$. Logo $f \notin \mathscr{C}$, ou seja, $\mathscr{C}$ não é cobertura. Portanto $I$ possui estratégia vencedora.

Vimos acima que todo espaço enumerável é de Rothberger. Podemos melhorar esse resultado com a seguinte Proposição:

\footnotetext{
${ }^{4}$ Observe que cada $F_{n, k}$ é aberto básico, pois $F_{n, k}=\prod_{m \in \omega} V_{m}$, onde $V_{n}=\{k\}$ e $V_{m}=2$ para $m \neq n$.
} 
Proposição 6.12. (MA) Seja $X$ de Lindelöf e $|X|<2^{\omega}$. Então $X$ é de Rothberger.

Demonstração. Vamos construir uma árvore baseado no jogo proposto. Seja $(T, \leq)$ onde para cada jogada de $I$ (podemos supor que cada cobertura é enumerável pois $X$ é de Lindelöf) a árvore bifurca em cada elemento da cobertura. E para cada bifurcação, $I$ faz a sua jogada escolhendo um elemento da cobertura. Para cada elemento escolhido por $I I$ o jogador $I$ responde com uma cobertura. Para esclarecer veja a figura:

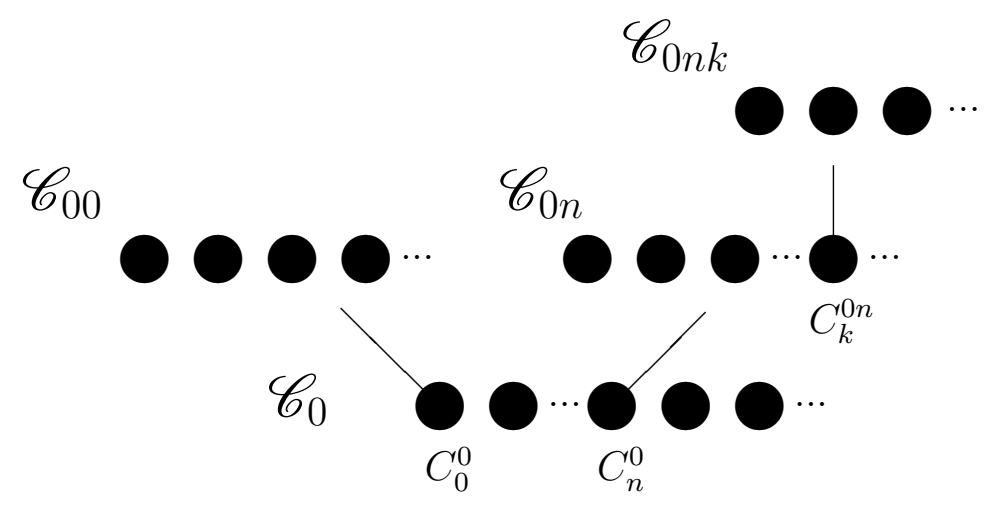

Figura 6.1: Observe que $I$ jogou com $\mathscr{C}_{0}$ então o jogador $I I$ pode escolher (por exemplo) o elemento $C_{0}^{0} \in \mathscr{C}_{0}$ e então o jogador $I$ responde com a cobertura $\mathscr{C}_{00}$. Ou se o jogador $I I$ escolher (por exemplo) o elemento $C_{n}^{0} \in \mathscr{C}_{0}$ o jogador $I$ responde com a cobertura $\mathscr{C}_{0 n}$. Daí digamos que $I I$ escolha $C_{k}^{0 n} \in \mathscr{C}_{0 n}$, então $I$ responderá a jogada com a cobertura $\mathscr{C}_{0 n k}$.

Pela maneira que $T$ foi concebida ela é enumerável e portanto satisfaz c.c.c..

Observe que se $F \subset T$ é um filtro então todos os seus elementos são comparáveis e, além disso, $F$ é fechado para baixo pela terceira condição de filtro. Portanto $F$ gera um ramo em $T$.

Seja $x \in X$ e $D_{x}=\{C \in \mathscr{C}: C \ni x$ e $\mathscr{C}$ cobertura dada por $I\}$. Temos $D_{x}$ é denso em $T$ pois para cada ponto $C \in T$ existe uma bifurcação acima e como tal bifurcação corresponde a uma cobertura dada por $I$ segue que existe um elemento $C^{\prime}$ que contém $x$ e $C \leq C^{\prime}$ na ordem da árvore. $\operatorname{Logo}\left(D_{x}\right)_{x \in X}$ é uma família de densos em $T$. Por $M A$ segue que existe $F \subset T$ filtro tal que $F \cap D_{x} \neq \emptyset$.

Pela contrução da árvore, $I I$ vence se existir um ramo de $T$ onde seus elementos formam uma cobertura para $X$. O filtro $F$ gera um ramo em $T$ que intercepta todos os 
elementos necessários para cobrir $X$. Portanto o jogador $I I$ vence.

\subsection{Jogo de Menger}

Em 1924, Menger introduziu a seguinte propriedade para um espaço métrico $X$ :

Para cada base $\mathcal{B}$ para a topologia de $X$, existem $B_{1}, B_{2}, \ldots$ elementos de $\mathcal{B}$ tais que $\lim _{n \rightarrow \infty} \operatorname{diam}\left(B_{n}\right)=0^{5}$, e $X=\bigcup_{n \in \omega} B_{n}$.

Depois disso, Hurewicz observou que a propriedade de Menger para bases poderia ser reformulada da seguinte maneira ${ }^{6}$ :

Para quaisquer coberturas abertas $\mathcal{U}_{1}, \mathcal{U}_{2}, \ldots$ de $X$, existem subfamílias finitas $\mathcal{F}_{1} \subset \mathcal{U}_{1}$, $\mathcal{F}_{2} \subset \mathcal{U}_{2}, \ldots$ tais que $\bigcup_{n \in \omega} \mathcal{F}_{n}$ é uma cobertura de $X$. E tal propriedade podia ser definida para qualquer $X$ (mesmo não métrico).

O jogo de Menger deriva-se dessa formulação proposta por Hurewicz.

Definição 6.13 (jogo de Menger). Considere o seguinte jogo entre os jogadores I e II - A cada rodada $n \in \omega$, o jogador I escolhe $\mathscr{C}_{n}$ uma cobertura aberta para X. Então o jogador II escolhe $C_{n} \subset \mathscr{C}_{n}$ finito. Dizemos que II vence o jogo se $\bigcup_{n<\omega} \cup C_{n}=X$.

Definição 6.14. Dizemos que X é um espaço de Menger se o jogador I não tem uma estratégia vencedora para o jogo de Menger.

Proposição 6.15. Todo espaço de Rothberger é de Menger. Todo espaço de Menger é de

\section{Lindelöf.}

Demonstração. A primeira parte é imediata da definição de espaço de Menger. Agora, seja $X$ um espaço de Menger e seja $\mathscr{C}$ uma cobertura aberta para $X$. O jogador $I$ jogará em todos os turnos $\mathscr{C}$. Assim, $I I$ jogará com $C_{n} \subset \mathscr{C}$ finito a cada turno. Como $X$ é de Menger, $I$ não tem estratégia vencedora, então temos que $\bigcup_{n \in \omega} \cup C_{n}=X$, tendo em vista que a escolha dos $C_{n}$ 's feito pelo jogador $I I$ testemunham o fato de $I$ não possuir estratégia vencedora. Como $\bigcup C_{n}$ é uma subcobertura enumerável de $\mathscr{C}$ concluimos que $X$ é Lindelöf.

\footnotetext{
${ }^{5}$ Seja $M$ um espaço métrico e $A \subset M$. Então $\operatorname{diam}(A)=\sup \{d(x, y): x, y \in A\}$.

${ }^{6}$ Para mais informações, veja [1].
} 
Como consequência, temos:

Corolário 6.16. (MA) Se $X$ é de Lindelöf e $|X|<2^{\omega}$, então X é de Menger.

Demonstração. Se $X$ é de Lindelöf e $|X|<2^{\omega}$ então pela Proposição 6.12Jogo de Rothbergerteorema.6.12 temos que $X$ é de Rothberger o que implica que $X$ é de Menger pela Proposição 6.15Jogo de Mengerteorema.6.15.

Proposição 6.17. O Jogo de Menger é equivalente à seguinte versão ${ }^{7}$ :

A cada rodada $k \in \omega$ o jogador I joga uma cobertura $\left(A_{n}^{k}\right)_{n \in \omega}$ tal que se $a<b$, então $A_{a}^{k} \subset A_{b}^{k}$

O jogador II escolhe um aberto da cobertura $\left(A_{n}^{k}\right)_{n \in \omega}$.

(jogam $\omega$ vezes)

O jogador II vence se os abertos escolhidos por ele formam uma cobertura.

Demonstração. Em primeiro lugar, suponha que as coberturas abertas jogadas sejam enumeráveis ${ }^{8}$. Sejam $I_{V}$ e $I I_{V}$ jogadores do jogo de Menger e sejam $I_{N}$ e $I I_{N}$ jogadores do jogo de Menger novo. Suponha que $I_{V}$ tenha estratégia vencedora. Seja $\left(B_{n}^{0}\right)_{n \in \omega}$ a primeira jogada de $I_{V}$. Agora no jogo novo, $I_{N}$ pega o que o $I_{V}$ jogou e adapta da seguinte maneira: Jogando $\left(A_{n}^{0}\right)_{n \in \omega}$, onde $A_{n}^{0}=\bigcup_{j \leq n} B_{j}^{0}{ }^{9}$. O jogador $I I_{N}$ faz a sua primeira jogada escolhendo, por exemplo, $A_{n_{0}}^{0}$. Essa jogada pode ser traduzida como uma jogada de $I I_{V}$ (por exemplo, $I I_{V}$ joga $\left\{B_{j}^{0}: j \leq n_{0}\right\}$ na primeira jogada) e suponha que $I I_{V}$ jogou essa adaptação. Em virtude da jogada feita por $I I_{V}$ o jogagor $I_{V}$ responde $\operatorname{com}\left(B_{n}^{1}\right)_{n \in \omega}$. Daí $I_{N}$ responde $I I_{N}$ com $\left(A_{n}^{1}\right)_{n \in \omega}$, onde $A_{n}^{1}=\bigcup_{j \leq n} B_{j}^{1}$. Digamos que $I I_{N}$ escolha $A_{n_{1}}^{1}$ e novamente suponha que $I I_{V}$ jogou $\left\{B_{j}^{0}: j \leq n_{1}\right\}$. E o jogo prossegue dessa maneira. Como o jogador $I_{V}$ usou uma estratégia vencedora implica que $\bigcup_{k \in \omega}\left(\bigcup_{j \leq n_{k}} B_{j}^{k}\right)$ não é uma cobertura aberta. Portanto $\bigcup_{k \in \omega} A_{n_{k}}^{k}$ não é cobertura aberta e com isso $I_{N}$ possui estratégia vencedora.

\footnotetext{
${ }^{7}$ Ser equivalente significa: $I$ tem estratégia vencedora num jogo se, e somente se, tem no outro (analogamente para $I I)$.

${ }^{8}$ Pode-se supor isso já que Menger $\Rightarrow$ Lindelöf.

${ }^{9} \mathrm{Com}$ isso, para cada $a<b \Rightarrow A_{a}^{0} \subset A_{b}^{0}$.
} 
Reciprocamente, suponha que $I_{N}$ tenha estratégia vencedora no segundo jogo. Seja $\left(A_{n}^{0}\right)_{n \in \omega}$ a primeira jogada de $I_{N}$. Então $I_{V}$ faz o seu primeiro movimento com $\left(A_{n}^{0}\right)_{n \in \omega}$. O jogador $I I_{V}$ faz o seu primeiro movimento $\left\{A_{00}^{0}, \ldots, A_{0 n_{0}}^{0}\right\}$. Sem perda de generalidade, podemos organizar os abertos de tal maneira $A_{00}^{0} \subset A_{01}^{0} \subset \cdots \subset A_{0 n_{0}}^{0}$. Então $I I_{N}$ faz a sua jogada com $A_{0 n_{0}}^{0}$. Agora, seja $\left(A_{n}^{1}\right)_{n \in \omega}$ a segunda jogada de $I_{N}$. Então $I_{V}$ faz o seu segundo movimento com $\left(A_{n}^{1}\right)_{n \in \omega}$. O jogador $I I_{V}$ faz a sua segunda jogada $\left\{A_{01}^{1}, \ldots, A_{1 n_{1}}^{1}\right\}$, novamente, sem perda de generalidade, podemos organizar os abertos de tal maneira $A_{10}^{0} \subset A_{11}^{0} \subset \cdots \subset A_{1 n_{1}}^{1}$. Então $I I_{N}$ joga com $A_{1 n_{1}}^{1}$. E assim por diante... Como $I_{N}$ tinha estratégia vencedora no segundo jogo segue que $\bigcup_{k \in \omega}\left\{A_{k 0}^{k}, \ldots, A_{k n_{k}}^{k}\right\}$ não forma uma cobertura aberta. Portanto $\bigcup_{k \in \omega} A_{k n_{k}}^{k}$ não forma uma cobertura aberta. Portanto $I_{V}$ possui estratégia vencedora.

Suponha que $I I_{V}$ tenha estratégia vencedora. No jogo de Menger novo, suponha que a primeira jogada de $I_{N}$ seja $\left(B_{n}^{0}\right)_{n \in \omega}$ (lembrando que para cada $a<b$ temos que $A_{a}^{0}<A_{b}^{0}$ ). Daí o jogador $I_{V}$ joga $\left(B_{n}^{0}\right)_{n \in \omega}$ na sua primeira jogada. Suponha que $I I_{V}$, na sua primeira jogada, tenha escolhido $\left\{B_{00}^{0}, \ldots, B_{0 n_{0}}^{0}\right\}$. Como $B_{00}^{0} \subset B_{01}^{0} \subset \cdots \subset B_{0 n_{0}}^{0}$, então $I I_{N}$ joga com $B_{0 n_{0}}^{0}$. Agora, suponha que a segunda jogada de $I_{N}$ seja $\left(B_{n}^{1}\right)_{n \in \omega}$. Novamente o jogador $I_{V}$ faz a sua jogada com $\left(B_{n}^{1}\right)_{n \in \omega}$ na sua segunda jogada. Suponha que $I I_{V}$, em sua segunda jogada, escolha $\left\{B_{10}^{1}, \ldots, B_{1 n_{1}}^{1}\right\}$. Como $B_{10}^{0} \subset B_{11}^{0} \subset \cdots \subset B_{1 n_{1}}^{1}$, então $I I_{N}$ joga com $B_{1 n_{1}}^{1}$. O jogo prossegue dessa maneira. Observe que $\bigcup_{k \in \omega}\left\{B_{k 0}^{k}, \ldots, B_{k n_{k}}^{k}\right\}$ forma uma cobertura aberta. Portanto $\bigcup_{k \in \omega} B_{k n_{k}}^{k}$ forma uma cobertura aberta e com isso $I I_{N}$ possui estratégia vencedora.

Reciprocamente, suponha que $I I_{N}$ tenha estratégia vencedora no segundo jogo. Seja $\left(B_{n}^{0}\right)_{n \in \omega}$ a primeira jogada de $I_{V}$. Agora $I_{N}$ joga com $\left(A_{n}^{0}\right)_{n \in \omega}$, onde $A_{n}^{0}=\bigcup_{j \leq n} B_{j}^{0}$. Então $I I_{N}$ responde com $A_{n_{0}}^{0}$. Daí $I I_{V}$ joga com $\left\{B_{j}^{0}: j \leq n_{0}\right\}$. Agora, seja $\left(B_{n}^{1}\right)_{n \in \omega}$ a segunda jogada de $I_{V}$. Agora $I_{N}$ joga $\left(A_{n}^{1}\right)_{n \in \omega}$, onde $A_{n}^{1}=\bigcup_{j<n} B_{j}^{1}$. Então $I I_{N}$ responde com $A_{n_{1}}^{1}$. Daí $I I_{V}$ joga com $\left\{B_{j}^{0}: j \leq n_{1}\right\}$. E assim por diante... Como $I I_{N}$ está usando uma estratégia vencedora, segue que $\bigcup_{k \in \omega} A_{k n_{k}}^{k}$ é uma cobertura. Portanto $\bigcup_{k \in \omega}\left(\bigcup_{j<n_{k}} B_{j}^{k}\right)$ forma uma cobertura e assim $I I_{V}$ possui estratégia vencedora.

Desse resultado extraimos o seguinte Corolário:

Corolário 6.18. (MA) Se $X$ é de Lindelöf e $X$ é a união que menos que contínuo 
compactos, então X é de Menger.

Demonstração. Considere o segundo jogo equivalente ao jogo de Menger (Proposição 6.17Jogo de Mengerteorema.6.17). Sejam $\left(K_{\xi}\right)_{\xi<\kappa}$ compactos para $\kappa<2^{\omega}$ onde $X=$ $\bigcup_{\xi<\kappa} K_{\xi}$. Podemos construir uma árvore das possíveis jogadas de $I(T, \leq)^{10}$ similar a Figura 6.12Jogo de Rothbergerteorema.6.12. Observe que se $\left(C_{n}\right)_{n \in \omega}$ é uma jogada de $I$ então para cada compacto $K_{\xi}$ existe $m \in \omega$ suficientemente grande tal que $K_{\xi} \subset C_{m}$. Seja $D_{\xi}=\left\{C \in \mathscr{C}: C \supset K_{\xi}\right.$ e $\mathscr{C}$ e cobertura dada por $\left.I\right\}$. Note que $D_{\xi}$ é denso em $T$, de fato seja $C \in T$ então acima de $C$ o jogador $I$ fará a sua jogada $\left(C_{n}^{\prime}\right)_{n \in \omega}$ e assim existirá $p \in \omega$ suficientemente grande tal que $K_{\xi} \subset C_{p}^{\prime}$ e $C \leq C_{p}^{\prime}$. Por $M A$ existe $F \subset T$ filtro tal que $F \cap D_{\xi} \neq \emptyset$, logo $\bigcup F \supset K_{\xi}$ para $\xi<\kappa$. Com isso temos a estratégia vencedora para o jogador $I I$.

Ao contrário do jogo de Rothberger, temos:

Proposição 6.19. Se X é compacto, então II tem estratégia vencedora para o jogo de Menger (e, portanto, $X$ é de Menger).

Demonstração. Seja $\mathscr{C}$ a primeira jogada de $I$. Então basta $I I$ jogar com a sub-cobertura finita de $\mathscr{C}$ que cobre $X$.

Na verdade podemos fazer ainda melhor:

Definição 6.20. Seja X um espaço topológico. Dizemos que X é um espaço $\sigma$-compacto se existem $\left(K_{n}\right)_{n \in \omega}$ compactos de $X$ tais que $X=\bigcup_{n \in \omega} K_{n}$.

Proposição 6.21. Se X é $\sigma$-compacto, então II tem estratégia vencedora no jogo de Menger.

Demonstração. Seja $X=\bigcup_{n \in \omega} K_{n}$ onde cada $K_{n}$ é compacto.

Seja $\mathscr{C}_{0}$ a primeira jogada de $I$ então $I I$ responde tomando a sub-coleção finita que cobre $K_{0}$. O jodador $I$ joga com $\mathscr{C}_{1}$ então o jogador $I I$ toma a sub-coleção que cobre $K_{1}$. No final de $\omega$ jogadas o jogador $I I$ cobre $\bigcup_{n \in \omega} K_{n}$.

\footnotetext{
${ }^{10}$ Note que $(T, \leq)$ satisfaz a c.c.c., pois $T$ é enumerável.
} 
Na seção anterior temos um exemplo de um espaço compacto que não é de Rothberger. Aqui temos o exemplo de um espaço de Lindelöf que não é de Menger.

Proposição 6.22. Existe um espaço de Lindelöf que não é de Menger. À saber $\omega^{\omega}$.

Demonstração. Em primeiro lugar, note que $\omega^{\omega}$ é de Lindelöf, pois $\omega^{\omega}$ possui base enumerável ${ }^{11}$. De fato, $\mathscr{B}=\left\{[s]: s \in \omega^{<\omega}\right\}$ onde $[s]=\left\{f \in \omega^{\omega}: s \subset f\right\}$ forma uma base enumerável para $\omega^{\omega}$ de maneira similar ao Lema 2.12Noções Básicas de Topologiateorema.2.12.

Seja $F_{n, k}=\{f: \omega \rightarrow \omega \mid f(n)=k\}^{12}$ e considere as seguintes jogadas feitas por $I$ :

$$
\begin{gathered}
\mathscr{C}_{0}=\left\{F_{0,0} ; F_{0,1} ; F_{0,2} ; F_{0,3} ; \ldots\right\}, \\
\mathscr{C}_{1}=\left\{F_{1,0} ; F_{1,1} ; F_{1,2} ; F_{1,3} ; \ldots\right\}, \\
\mathscr{C}_{2}=\left\{F_{2,0} ; F_{2,1} ; F_{2,2} ; F_{2,3} ; \ldots\right\}, \\
\vdots \\
\mathscr{C}_{n}=\left\{F_{n, 0} ; F_{n, 1} ; F_{n, 2} ; F_{n, 3} ; \ldots\right\},
\end{gathered}
$$

O jogador II no seu primeiro movimento escolhe uma quantidade finita de elementos de $\mathscr{C}_{0}$. Na segunda jogada de $I I$ escolhe uma quantidade finita de elementos de $\mathscr{C}_{1}$. Repetindo o mesmo processo nas jogadas seguintes. Tome $f \in \omega^{\omega}$ definida como $f(n)=k$ onde $F_{n, k}$ não pertença a coleção finita que $I I$ jogou. Logo, $I I$ não cobre $f$ e portanto $\omega^{\omega}$ não é de Menger.

\footnotetext{
${ }^{11}$ Seja $\mathscr{C}$ uma cobertura de $\omega^{\omega}$ e seja $f \in \omega^{\omega}$ onde $f \in C \in \mathscr{C}$. Então existe $B_{n} \in \mathscr{B}$ ( $\mathscr{B}$ base enumerável para o topologia de $\left.\omega^{\omega}\right)$ tal que $f \in B_{n} \subset C$.

${ }^{12}$ Observe que cada $F_{n, k}$ é aberto básico para a topologia de $\omega^{\omega}$.
} 


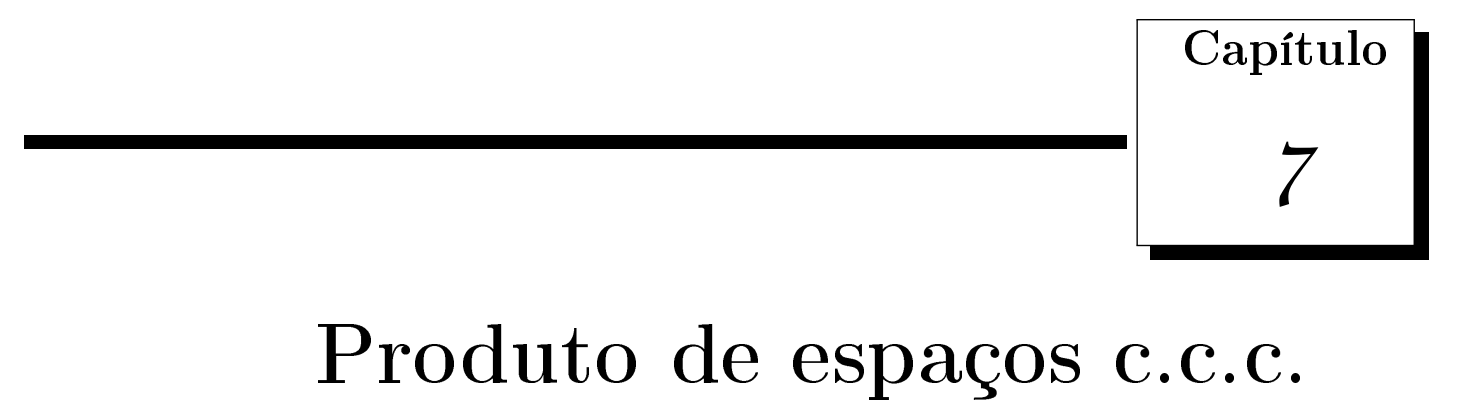

Considere a seguinte Proposição:

Proposição 7.1. Se um espaço topológico é separável então satisfaz c.c.c.. Porém a recíproca não é verdadeira, isto é, existe um espaço que é c.c.c. mas não é separável.

Demonstração. Seja $X$ um espaço topológico separável e seja $\left\{U_{\alpha}: \alpha<\omega_{1}\right\}$ uma família não enumerável de abertos não vazios dois a dois disjuntos. Por $X$ ser separável existe um subconjunto $D \subset X$ enumerável e denso. Então cada $U_{\alpha}$ intercepta um elemento distinto de $D$ e isso contradiz o fato de $D$ ser enumerável.

A prova de que a recíproca não é verdadeira será mostrada na Proposição 7.5Sob ZFC teorema.7.5 da próxima seção.

Como de costume, procuramos algumas consequências após definir algo. Vejamos que o produto de espaços separáveis é separável

Proposição 7.2. Produto finito de espaços separáveis é separável.

Demonstração. Faremos a demonstração para o produto de dois espaços. Para o produto finito qualquer, basta proceder por indução. Sejam $X$ e $Y$ espaços separáveis, i.e., existem $A \subset X$ e $B \subset Y$ subconjuntos densos e enumeráveis. Agora, note que $A \times B$ é enumerável e afirmamos que é denso em $X \times Y$. De fato, seja um aberto básico não vazio $U=V \times Z$, 
onde $V$ é aberto não vazio em $X$ e $Z$ é aberto não vazio em $Y$. Então existem pontos $a \in A$ e $b \in B$ tais que $a \in V$ e $b \in Z$ e, portanto, $(a, b) \in U$.

Disso concluimos que o produto finito de espaços separáveis satisfaz a c.c.c.. Porém, poderíamos nos perguntar se o produto de espaços que satisfaz a c.c.c. é c.c.c.. O que nos será respondido nas duas próximas seções será que o produto de espaços c.c.c. ser c.c.c. é consistente com $Z F C$ e que o produto de espaços c.c.c. não ser c.c.c. também é consistente com $Z F C$.

\subsection{Sob $Z F C$}

Faremos alguns resultados que não necessitam de axiomas extras. Começaremos enunciando uma ferramenta muito importante em teoria de conjuntos, o $\Delta$-sistema:

Lema 7.3 (Lema do $\Delta$ - sistema). Seja $\mathcal{F}$ uma família não enumerável de conjuntos finitos. Então existem $\Delta$ um conjunto finito $e \mathcal{G} \subset \mathcal{F}$ não enumerável tais que, para todos $A, B \in \mathcal{G}$ distintos, $A \cap B=\Delta$. Neste caso, dizemos que $\mathcal{G}$ forma um $\Delta$-sistema de raíz $\Delta$.

Demonstração. Seja $\mathcal{F}$ uma família não enumerável de conjuntos finitos . Então podemos supor que para todo $X \in \mathcal{F}$ temos que $|X|=n$ para algum $n \in \omega$. De fato, seja $\mathcal{F}_{0}=\{A \in \mathcal{F}:|A|=0\}, \mathcal{F}_{1}=\{A \in \mathcal{F}:|A|=1\}, \ldots, \mathcal{F}_{n}=\{A \in \mathcal{F}:|A|=n\}, \ldots$

Assim

$$
\mathcal{F}=\bigcup_{n \in \omega} \mathcal{F}_{n}
$$

Suponha que não exista uma família uma $\mathcal{F}_{n_{0}}$ que seja não enumerável. Então todos os $\mathcal{F}_{n}$ 's são enumeráveis e portanto $\mathcal{F}$ é enumerável o que é um absurdo.

Vamos provar o resultado por indução sobre $n$.

Suponha que $n=1$. Seja $\mathcal{F}$ uma família não enumerável tal que seus elementos tem cardinalidade igual a 1 . Então, para $X, Y \in \mathcal{F}$ distintos, segue que $X \cap Y=\emptyset$ e portanto $\Delta=\emptyset$.

Agora, mostremos que o resultado vale para uma família não enumerável $\mathcal{F}$ de conjuntos de cardinalidade $n+1$, supondo que vale para qualquer família não enumerável de 
conjuntos de cardinalidade $n$.

Vejamos os seguintes casos:

(1) Suponha que exista algum $a$ tal que $a$ pertença a uma quantidade não enumerável de elementos $\mathcal{F}$, onde esses elementos possuem cardinalidade $n+1$. Seja $\mathcal{C}=\{A \in$ $\mathcal{F}: a \in A\}$ e defina $\mathcal{C}-\{a\}=\{A \backslash\{a\}: A \in \mathcal{C}\}$. Aplicando a hipótese de indução sobre esse conjunto obtemos $\mathcal{G}^{\prime} \subset \mathcal{C}-\{a\}$ não enumerável e $\Delta^{\prime}$ um conjunto onde $A \cap B=\Delta^{\prime}$ para $A, B \in \mathcal{G}^{\prime}$ distintos. Assim $\mathcal{G}=\left\{A \cup\{a\}: A \in \mathcal{G}^{\prime}\right\}$ e $\Delta=\Delta^{\prime} \cup\{a\}$.

(2) Suponha que não exista tal a que pertença a uma quantidade não enumerável de elementos de $\mathcal{F}$. Então, fixado $a$, a quantidade de elementos que contém $a$ é no máximo enumerável.

Seja $A_{0} \in \mathcal{F}$. Escreva $A_{0}=\left\{a_{0}, \ldots, a_{n}\right\}$. Sejam $\mathcal{F}_{a_{0}}=\left\{A \in \mathcal{F}: a_{0} \in A\right\}, \mathcal{F}_{a_{1}}=\{A \in$ $\left.\mathcal{F}: a_{1} \in A\right\}, \ldots, \mathcal{F}_{a_{n}}=\left\{A \in \mathcal{F}: a_{n} \in A\right\}$. Como $\mathcal{F}$ é não enumerável, e cada $\mathcal{F}_{a_{i}}$ é enumerável, temos que $\mathcal{F} \backslash\left(\mathcal{F}_{a_{0}} \cup \cdots \cup \mathcal{F}_{a_{n}}\right)$ é não enumerável.

Agora, tome $A_{1} \in \mathcal{F} \backslash\left(\mathcal{F}_{a_{0}} \cup \cdots \cup \mathcal{F}_{a_{n}}\right)$ e considere $\mathcal{F} \backslash\left(\bigcup_{a \in\left(A_{0} \cup A_{1}\right)} \mathcal{F}_{a}\right)$. Novamente temos que $\mathcal{F} \backslash\left(\bigcup_{a \in\left(A_{0} \cup A_{1}\right)} \mathcal{F}_{a}\right)$ é não enumerável. Tome $A_{2} \in \mathcal{F} \backslash\left(\bigcup_{a \in\left(A_{0} \cup A_{1}\right)} \mathcal{F}_{a}\right)$ e observe que $A_{0} \cap A_{1}=\emptyset$.

Seja $\xi<\omega_{1}$. Considere $\mathcal{F} \backslash\left(\bigcup_{a \in\left(\cup_{\eta<\xi} A_{\eta}\right)} \mathcal{F}_{a}\right)$ e note que tal conjunto é não enumerável. De fato, como $\xi$ é enumerável e para cada $\eta<\xi$ temos que $\left|A_{\eta}\right|=n+1$, $\operatorname{logo} \bigcup_{\eta<\xi} A_{\eta}$ é enumerável. Como cada $\mathcal{F}_{a}$ é enumerável segue que $\bigcup_{a \in\left(\bigcup_{\eta<\xi} A_{\eta}\right)} \mathcal{F}_{a}$ é enumerável e, portanto, $\mathcal{F} \backslash\left(\bigcup_{a \in\left(\bigcup_{\eta<\xi} A_{\eta}\right)} \mathcal{F}_{a}\right)$ é não enumerável. Agora, tome $A_{\xi} \in \mathcal{F} \backslash\left(\bigcup_{a \in\left(\bigcup_{\eta<\xi} A_{\eta}\right)} \mathcal{F}_{a}\right)$ e note que $A_{\xi} \cap\left(\bigcup_{\eta<\xi} A_{\eta}\right)=\emptyset$.

Com isso obtemos $\left\{A_{\xi}: \xi<\omega_{1}\right\}$ onde são dois a dois disjuntos. Portanto $\Delta=\emptyset$.

O próximo teorema é um resultado muito curioso. Pois, dado o produto de espaços c.c.c. basta que o produto de uma parte finita seja c.c.c. para que o produto todo satisfaça essa propriedade.

Teorema 7.4. Suponha que $X_{i}$ para $i \in I$ são espaços tais que para todo $r \subset I$ finito, $\prod_{i \in r} X_{i}$ é c.c.c. Então $\prod_{i \in I} X_{i}$ é c.c.c. 
Demonstração. Suponha que $\prod_{i \in I} X_{i}$ não satisfaça c.c.c.. Então existe uma família $\left(U_{\alpha}\right)_{\alpha<\omega_{1}}$ de abertos não vazios dois a dois disjuntos. Tome um aberto básico não vazio contido em $U_{\alpha}$. Renomeie para $U_{\alpha}$ esses abertos básicos. Note que para cada $\alpha$ temos $a_{\alpha}$ finito tal que $U_{\alpha}=\prod_{i \in I} V_{i}^{\alpha}$, onde $V_{i}^{\alpha}$ são abertos de $X_{i}$ para $i \in a_{\alpha}$, para os demais, $V_{i}^{\alpha}=X_{i}$

Vamos aplicar o Lema do $\Delta$-sistema em $\left\{a_{\alpha}: \alpha<\omega_{1}\right\}$.

Porém nada impede que dado $a_{\gamma}$ exista $\beta<\omega_{1}$ tal que $a_{\beta}=a_{\gamma} \operatorname{com} \beta \neq \gamma$, mas mesmo assim podemos usar o $\Delta$-sistema. Mostremos o por quê:

- Suponha que exista $a$ tal que $a=a_{\alpha}$ para todo $\alpha<\omega_{1}$. Seja $\pi_{a}\left[U_{\alpha}\right]=\prod_{i \in a} V_{i}^{\alpha}$ a projeção de $U_{\alpha}$ em $\prod_{i \in a} X_{i}$. Note que, para $\alpha \neq \beta$, temos que $\pi_{a}\left[U_{\alpha}\right] \cap \pi_{a}\left[U_{\beta}\right]=\emptyset$. Então $\left(\pi_{a}\left[U_{\alpha}\right]\right)_{\alpha<\omega_{1}}$ é uma anticadeia em $\prod_{i \in a} X_{i}$. Contradição com o fato de $\prod_{i \in a} X_{i}$ ser c.c.c..

- Agora, se $\left\{a_{\alpha}: \alpha<\omega_{1}\right\}$ for finito $\left(\left\{b_{1}, \ldots, b_{n}\right\}=\left\{a_{\alpha}: \alpha<\omega_{1}\right\}\right)$. Então, uma quantidade não enumerável de elementos de $\left\{a_{\alpha}: \alpha<\omega_{1}\right\}$ será igual a $b_{k}$ para algum $1 \leq k \leq n$. E assim, repetindo o mesmo argumento feito para o caso unitário teremos uma contradição.

- Finalmente, se $\left\{a_{\alpha}: \alpha<\omega_{1}\right\}$ for enumerável, teremos também uma contradição de maneira análoga ao que foi feito anteriormente, pois algum dos $a_{\alpha}$ se repetirá uma quantidade não-enumerável de vezes.

Assim, aplicando o Lema do $\Delta$-sistema existe $A \subset \omega_{1}$ não enumerável tal que $\left\{a_{\alpha}\right.$ : $\alpha \in A\}$ forma um $\Delta$-sistema com raiz $r$.

Note que $r \neq \emptyset$, pois, se $a_{\alpha} \cap a_{\beta}=\emptyset$ então $U_{\alpha} \cap U_{\beta} \neq \emptyset$. De fato, seja $z=\left(z_{j}\right)_{j \in I} \in$ $\prod_{i \in I} X_{i}$. Seja $x_{1}=\left(x_{j}^{1}\right)_{j \in I} \in U_{\alpha}$. Então $x_{j}^{1} \in V_{j}^{\alpha}$ para todo $j \in a_{\alpha}$ e seja $x_{2}=\left(x_{j}^{2}\right)_{j \in I} \in$ $U_{\beta}$. Então $x_{j}^{2} \in V_{j}^{\beta}$ para todo $j \in a_{\beta}$. Defina

$$
y=\left(y_{j}\right)_{j \in I}= \begin{cases}y_{j}=z_{j} & \text { se } j \notin a_{\alpha} \cup a_{\beta}, \\ y_{j}=x_{j}^{1} & \text { se } j \in a_{\alpha}, \\ y_{j}=x_{j}^{2} & \text { se } j \in a_{\beta} .\end{cases}
$$


Logo $U_{\alpha} \cap U_{\beta} \neq \emptyset$, pois $y \in U_{\alpha} \cap U_{\beta}$, contradizendo a hipótese.

Seja $\pi_{r}\left(U_{\alpha}\right)$ a projeção de $U_{\alpha}$ em $\prod_{i \in r} X_{i}$. Então $\pi_{r}\left(U_{\alpha}\right)$ para cada $\alpha \in A$ forma uma família não enumerável de abertos disjuntos em $\prod_{i \in r} X_{i}$. Pois, suponha que existam $\alpha \neq \beta$ tal que $\pi_{r}\left(U_{\alpha}\right) \cap \pi_{r}\left(U_{\beta}\right) \neq \emptyset$. Considere a seguinte partição de $I=r \cup\left(a_{\alpha} \backslash r\right) \cup\left(a_{\beta} \backslash\right.$ $r) \cup\left[I \backslash\left(a_{\alpha} \cup a_{\beta}\right)\right]$. Seja $x=\left(x_{j}\right)_{j \in I} \in \pi_{r}\left(U_{\alpha}\right) \cap \pi_{r}\left(U_{\beta}\right)$ então $x_{j} \in\left(\prod_{i \in r} V_{i}^{\alpha}\right) \cap\left(\prod_{i \in r} V_{i}^{\beta}\right)$ para $j \in r$. Sejam $y^{\prime}=\left(y_{j}^{\prime}\right)_{j \in I} \in U_{\alpha}$ e $y^{\prime \prime}=\left(y_{j}^{\prime \prime}\right)_{j \in I} \in U_{\beta}$. Tome

$$
y=\left(y_{j}\right)_{j \in I}=\left\{\begin{array}{l}
y_{j}=x_{j} \quad \text { se } j \in r \cup\left[I \backslash\left(a_{\alpha} \cup a_{\beta}\right)\right], \\
y_{j}=y_{j}^{\prime} \quad \text { se } j \in a_{\alpha} \backslash r, \\
y_{j}=y_{j}^{\prime \prime} \quad \text { se } j \in a_{\beta} \backslash r .
\end{array}\right.
$$

então $y \in U_{\alpha} \cap U_{\beta}$. Portanto se $U_{\alpha} \cap U_{\beta}=\emptyset$ implica que $\pi_{r}\left(U_{\alpha}\right) \cap\left(\pi_{r}\left(U_{\beta}\right)=\emptyset\right.$.

Agora estamos em condições de mostrar a invalidade da recíproca da Proposição 7.1Produto de espaços c.c.cteorema.7.1.

Proposição 7.5. Seja $\kappa>2^{\omega}$, então o espaço $2^{\kappa}$ não é separável. Porém $2^{\kappa}$ é c.c.c..

Demonstração. Suponha que $2^{\kappa}$ seja separável. Seja $D \subset 2^{\kappa}$ um subconjunto enumerável denso. Para cada $\xi<\kappa$ defina $D_{\xi}=\left\{f \in 2^{\kappa}: f(\xi)=0\right\} \cap D$. Observe que cada $D_{\xi}$ é não vazio pois $\left\{f \in 2^{\kappa}: f(\xi)=0\right\}$ é aberto não vazio. Observe que se $\alpha, \beta<\kappa \operatorname{com} \alpha \neq \beta$ então $D_{\alpha} \neq D_{\beta}{ }^{1}$. Com isso temos uma função injetora de $\kappa$ em $\wp(D)$ e então $\kappa \leq 2^{\omega}$ o que é um absurdo. Portanto $2^{\kappa}$ não é separável.

Note que o produto finito $2 \times 2 \times \cdots 2 \times 2$ é c.c.c.. Então, pelo Teorema 7.4Sob $Z F C$ teorema.7.4, temos que $2^{\kappa}$ é c.c.c.

\subsection{Sob o Axioma de Martin}

Mostraremos que se assumirmos $M A$ então teremos que produto de c.c.c. é c.c.c..

\footnotetext{
${ }^{1}$ Sejam $\tilde{D}_{\alpha}=\left\{f \in 2^{\kappa}: f(\alpha)=0\right\}, \tilde{D}_{\beta}=\left\{f \in 2^{\kappa}: f(\beta)=0\right\}, E_{\alpha}=\left\{f \in 2^{\kappa}: f(\alpha)=1\right\} \mathrm{e}$ $E_{\beta}=\left\{f \in 2^{\kappa}: f(\beta)=1\right\}$. Observe que $\tilde{D}_{\alpha}, \tilde{D}_{\beta}, E_{\alpha}$ e $E_{\beta}$ são abertos. Daí. tome $f \in \tilde{D}_{\alpha} \cap E_{\beta} \cap D$ e tome $h \in \tilde{D}_{\beta} \cap E_{\alpha} \cap D$. Então $f \in D_{\alpha}$ e $f \notin D_{\beta}$, assim como $h \in D_{\beta}$ e $h \notin D_{\alpha}$.
} 
Lema $7.6(M A+\neg C H)$. Seja $X$ c.c.c. $e\left\{U_{\alpha}: \alpha<\omega_{1}\right\}$ uma familia não vazia de subconjuntos abertos de $X$, então existe um subconjunto $A \subset \omega_{1}$ não enumerável tal que $\left\{U_{\alpha}: \alpha \in \alpha\right\}$ possui a propriedade da intersecção finita.

Demonstração. Seja $V_{\alpha}=\bigcup_{\gamma>\alpha} U_{\gamma}$. Note que, para $\alpha<\beta$, temos $V_{\beta} \subset V_{\alpha}$.

Afirmamos que existe $\alpha<\omega_{1}$ tal que para todo $\beta>\alpha$ temos que $\overline{V_{\beta}}=\overline{V_{\alpha}}$. Suponha que não exista tal $\alpha$. Então teríamos uma sequência crescente de ordinais $a_{\xi} \operatorname{com} \xi<\omega_{1}$ tal que para cada $\xi$ temos $\overline{V_{a \xi+1}} \neq \overline{V_{a \xi}}$ onde $A_{a \xi}=V_{a \xi} \backslash \overline{V_{a \xi+1}} \neq \emptyset$. De fato, seja $x \in \overline{V_{a \xi}}$ como $\overline{V_{a \xi+1}} \varsubsetneqq \overline{V_{a \xi}}$ existe um aberto $V \ni x$ tal que $V \cap V_{a \xi} \neq \emptyset$ e $V \cap V_{a \xi+1}=\emptyset$, daí tome $y \in V \cap V_{a \xi}$ então $y \in V_{a \xi}$ e $y \notin \overline{V_{a \xi+1}}$. Esses abertos $\left\{A_{a \xi}\right\}_{\xi<\omega_{1}}$ formam uma família não enumerável de abertos disjuntos que contradiz a hipótese de $X$ ser c.c.c..

Agora fixe esse $\alpha$ satisfazendo a afirmação anterior e considere o seguinte conjunto: $\mathbb{P}=\left\{P \subset V_{\alpha}: p\right.$ é aberto e $\left.P \neq \emptyset\right\}$. Note que $(\mathbb{P}, \subset)$ é c.c.c. por que $X$ o é ${ }^{2}$.

Defina $D_{\beta}=\left\{P \in \mathbb{P}:\right.$ exista $\gamma>\beta$ tal que $\left.P \subset U_{\gamma}\right\}$. $D_{\beta}$ é denso em $\mathbb{P}$, pois seja $P \in \mathbb{P}$ então $P \subset V_{\alpha} \subset \overline{V_{\alpha}}=\overline{V_{\beta}}$ e daí $P \cap V_{\beta} \neq \emptyset$ e com isso $P \cap U_{\gamma} \neq \emptyset$ para algum $\gamma>\beta$.

Temos que $\left\{D_{\beta}\right\}_{\beta<\omega_{1}}$ é uma família de abertos densos em $\mathbb{P}$ e então assumindo o Axioma de Martin temos que existe um filtro $G \subset \mathbb{P}$ tal que $G \cap D_{\beta} \neq \emptyset$. Assim, defina $A=\left\{\gamma<\omega_{1}\right.$ : exista $P \in G$ tal que $\left.P \subset U_{\gamma}\right\}$ e então $\left\{U_{\gamma}: \gamma \in A\right\}$ possui a propriedade da intersecção finita.

Enfim, o teorema desejado.

Teorema 7.7 (MA). O produto de espaços c.c.c. é um espaço c.c.c..

Demonstração. É suficiente mostrar que o produto de dois espaços c.c.c. é c.c.c. e daí aplicando o Teorema 7.4Sob $Z F C$ teorema.7.4 o resultado segue.

Afirmação 7.8 (MA). Sejam $X$ e $Y$ espaços c.c.c., então $X \times Y$ é c.c.c..

Demonstração. Suponha que $X \times Y$ não é c.c.c.. Seja $\left\{W_{\alpha}: \alpha<\omega_{1}\right\}$ uma família de abertos não vazios dois a dois disjuntos em $X \times Y$. Para cada $\alpha$ considere um aberto básico não vazio $U_{\alpha} \times V_{\alpha} \subset W_{\alpha}$. Pelo lema anterior seja $A \subset \omega_{1}$ um conjunto não enumerável

\footnotetext{
${ }^{2}$ No sentido de espaço topológico.
} 
tal que a família $\left\{U_{\alpha}: \alpha \in A\right\}$ tem p.i.f. Se $\alpha, \beta \in A$ e $\alpha \neq \beta$, então $U_{\alpha} \cap U_{\beta} \neq \emptyset$. Mas $\left(U_{\alpha} \times V_{\alpha}\right) \cap\left(U_{\beta} \times V_{\beta}\right)=\left(U_{\alpha} \cap U_{\beta}\right) \times\left(V_{\alpha} \cap V_{\beta}\right)=\emptyset$ e portanto $V_{\alpha} \cap V_{\beta}=\emptyset$. Logo, os elementos da família $\left\{V_{\alpha}: \alpha \in A\right\}$ são dois a dois disjuntos contrarianto o fato de $Y$ ser c.c.c.

\subsection{Sob a negação da Hipótese de Suslin}

Na seção 5.2MA implica $S H$ section.5.2 do capítulo 5A Hipótese de Suslinchapter.5 foi mostrado que $M A$ implica $S H$, mostrando que $M A$ implica que não existe árvore de Suslin e que a existência de uma árvore de Suslin é equivalente a existência de uma reta de Suslin. Agora mostraremos o mesmo resultado usando o fato do Axioma de Martin implicar que o produto de c.c.c. é c.c.c.. Por fim extrairemos que $\neg S H$ implica que o quadrado de espaços c.c.c. não é c.c.c..

Teorema 7.9. MA implica $S H$.

Demonstração. Sabemos que $M A$ implica que o produto de espaços c.c.c. é c.c.c.. Então, o Teorema é imediato se assumirmos o seguinte lema:

Lema 7.10. Se X é uma reta de Suslin, então $X^{2}$ não é c.c.c.

Demonstração. Se $a, b \in X$ e $a<b$, seja $] a, b[$ o intervalo aberto $\{x \in X: a<x<b\}$. O intervalo $] a, b[$ pode ser vazio se $a$ e $b$ são adjacentes $(a=b)$.

Por indução em $\alpha<\omega_{1}$, vamos encontrar $a_{\alpha}, b_{\alpha}, c_{\alpha} \in X$ tal que

(1) $a_{\alpha}<b_{\alpha}<c_{\alpha}$

(2) $] a_{\alpha}, b_{\alpha}[\neq \emptyset \mathrm{e}] b_{\alpha}, c_{\alpha}[\neq \emptyset$;

(3) $] a_{\alpha}, c_{\alpha}\left[\cap\left\{b_{\xi}: \xi<\alpha\right\}=\emptyset\right.$.

Assumindo essas condições satisfeitas, seja $\left.U_{\alpha}=\right] a_{\alpha}, b_{\alpha}[\times] b_{\alpha}, c_{\alpha}[$, por (2) note que $U_{\alpha} \neq \emptyset$. 
Se $\xi<\alpha$, então $U_{\xi} \cap U_{\alpha}=\emptyset$. De fato, por (3) ou $b_{\xi} \leq a_{\alpha}$ implica que $] a_{\xi}, b_{\xi}[\cap] a_{\alpha}, b_{\alpha}[=\emptyset$, ou $b_{\xi} \geq c_{\alpha}$ implica que $] b_{\xi}, c_{\xi}[\cap] b_{\alpha}, c_{\alpha}\left[=\emptyset\right.$. Portanto (]$a_{\xi}, b_{\xi}[\times] b_{\xi}, c_{\xi}[) \cap(] a_{\alpha}, b_{\alpha}[\times] b_{\alpha}, c_{\alpha}[)=$ $\emptyset$. Assim, $\left\{U_{\alpha}: \alpha<\omega_{1}\right\}$ refuta a condição de $X^{2}$ ser c.c.c..

Para encontrar $a_{\alpha}, b_{\alpha}, c_{\alpha} \in X$. Seja $W$ o conjunto de todos os pontos isolados de $X$. como cada ponto isolado é aberto e $X$ é c.c.c. segue que $|W| \leq \omega$.

Agora, assuma tomados os pontos $a_{\xi}, b_{\xi}, c_{\xi}$ para $\xi<\alpha$. Como $X$ não é separável implica que $X \backslash \overline{\left(W \cup\left\{b_{\xi}: \xi<\alpha\right\}\right)} \neq \emptyset$, pois $\left|\left\{b_{\xi}: \xi<\alpha\right\}\right| \leq \omega$. Por $X \backslash \overline{\left(W \cup\left\{b_{\xi}: \xi<\alpha\right\}\right)}$ ser aberto, contém um intervalo não vazio $] a_{\alpha}, c_{\alpha}[$. Como $] a_{\alpha}, c_{\alpha}[$ não contém pontos isolados e é infinito então podemos escolher $\left.b_{\alpha} \in\right] a_{\alpha}, c_{\alpha}[$ tal que $] a_{\alpha}, b_{\alpha}[\neq \emptyset \mathrm{e}] b_{\alpha}, c_{\alpha}[\neq \emptyset$.

Vamos analisar melhor esse último argumento.

(a) Suponha que para todo $b \in] a_{\alpha}, c_{\alpha}[$ implica que $] a_{\alpha}, b[=\emptyset \mathrm{e}] b, c_{\alpha}[=\emptyset$. Logo $] a_{\alpha}, c_{\alpha}[=$ $\{b\}$ e portanto $b$ é isolado. Logo, pelo menos um intervalo é não vazio.

(b) Suponha que para todo $\left.b^{\prime} \in\right] a_{\alpha}, c_{\alpha}[$ temos as seguintes possibilidades: ou $] a_{\alpha}, b^{\prime}[=\emptyset$ ou $] b^{\prime}, c_{\alpha}[=\emptyset$. Suponha, sem perda de generalidade, que $] a_{\alpha}, b^{\prime}[\neq \emptyset$, então existe $\left.b^{\prime \prime} \in\right] a_{\alpha}, b^{\prime}[$. Daí pelo caso $(a)$ ou $] a_{\alpha}, b^{\prime \prime}[\neq \emptyset$ ou $] b^{\prime \prime}, b^{\prime}[\neq \emptyset$.

(i) Se $] a_{\alpha}, b^{\prime \prime}\left[\neq \emptyset\right.$ tome $b_{\alpha}=b^{\prime \prime}$, então $] a_{\alpha}, b_{\alpha}[\neq \emptyset$ e $] b_{\alpha}, c_{\alpha}[\neq \emptyset$.

(ii) Se $] b^{\prime \prime}, b^{\prime}\left[\neq \emptyset\right.$, tome $\left.b_{\alpha} \in\right] b^{\prime \prime}, b^{\prime}[$, então $] a_{\alpha}, b_{\alpha}[\neq \emptyset$ e $] b_{\alpha}, c_{\alpha}[\neq \emptyset$. Portanto existe $\left.b_{\alpha} \in\right] a_{\alpha}, c_{\alpha}[$ tal que $] a_{\alpha}, b_{\alpha}[\neq \emptyset$ e $] b_{\alpha}, c_{\alpha}[\neq \emptyset$.

Com isso temos a consistência de que produto de espaços c.c.c. não é c.c.c..

Corolário 7.11. $\neg S H$ implica que o quadrado de espaços c.c.c. não necessariamente é c.c.c. 


\section{Capítulo}

8

\section{O espaço de Stone de uma álgebra de}

\section{Boole}

O objetivo desse capítulo é construir um espaço compacto Hausdorff, onde o conjunto dos naturais seja denso e onde toda função $f: \omega \rightarrow[0,1]$ possa ser estendida continuamente. Faremos isso em três etapas:

Na primeira seção definiremos o espaço de Stone de uma álgebra de Boole e veremos que tal espaço no fornece algumas propriedades interessantes, como por exemplo, ser compacto e Hausdorff.

Na segunda, trataremos da convergência de ultrafiltros e daremos uma aplicação em uma demostração do Teorema de Tychonoff usando tal conceito.

Finalmente, na terceira seção, construiremos o espaço de Stone-Čech dos naturais usando ultrafiltros e daremos uma aplicação dessa construção em análise funcional.

\subsection{Espaço de Stone}

Definição 8.1. Seja A uma álgebra de Boole e considere Ult $(A)=\{u \subset A: u$ é ultrafiltro de $A\})$. Para cada $a \in A$ consideremos:

$$
a^{*}=\{u \in U l t(A): a \in u\}
$$


i.e., $a \in u$ se, e somente, se $u \in a^{*}$.

Vamos considerar $\operatorname{Ult}(A) \operatorname{com}\left\{a^{*}: a \in A\right\}$ como base para a topologia ${ }^{1}$. Tal espaço chama-se espaço de Stone de $A$ e o denotaremos por $s(A)$.

Com a seguinte definição e o próximo teorema, veremos algumas propriedades operacionais que os elementos de $\left\{a^{*}: a \in A\right\}$ satisfazem.

Definição 8.2. Chamamos de Corpo de Conjuntos (subconjuntos de X) uma familia de conjunto que é uma álgebra de Boole com as operações usuais

Teorema 8.3 (Representação de Stone (versão conjuntista)). Toda álgebra de Boole é isomorfa a um corpo de conjuntos.

Demonstração. Considere

$$
\begin{aligned}
h: A & \rightarrow \wp(U l t(A)) \\
a & \mapsto h(a)=a^{*}
\end{aligned}
$$

Vamos mostrar que $h$ é um homomorfismo injetor. Sejam $a, b \in A$.

- $a^{*} b^{*}=a^{*} \cap b^{*}=\{u \in U l t(A): a \in u\} \cap\{u \in U l t(A): b \in u\}=\{u \in U l t(A): a, b \in$ $u\}=(a b)^{*}$;

- $(-a)^{*}=\{u \in U l t(A):-a \in u\}=\{u \in U l t(A): a \notin u\}=U l t(A) \backslash\{u \in \operatorname{Ult}(A)$ : $a \in u\}=-(a)^{*}$

- $a^{*}+b^{*}=a^{*} \cup b^{*}=\{u \in U l t(A): a \in u$ ou $b \in u\}=\{u \in \operatorname{Ult}(A): a+b \in u\}=$ $(a+b)^{*}$, pois $u$ é primo ${ }^{2}$;

- $1^{*}=U l t(A)$;

- $0^{*}=\emptyset$.

Temos ainda que $h$ é injetora, pois dados $a, b \in A$ com $a \neq b$ temos que existe $u \in U l t(A)$ tal que $a \in u$ e $b \notin u$ ou $a \notin u$ e $b \in u$.

\footnotetext{
${ }^{1}$ Seja $u \in U l t(A)$. Seja $a \in u(u \neq \emptyset)$ e considere $a^{*}$ então $u \in a^{*}$. Agora, seja $u \in a^{*} \cap b^{*}$, então $a \in u$ e $b \in u$. Assim $a b \in u$ e então $u \in(a b)^{*}$.

${ }^{2} u$ é primo se $x+y \in u$ temos $x \in u$ ou $y \in u$.
} 
O espaço $U l t(A)$, depois dessa formulação um pouco complicada, é um espaço bem rico, pois tal espaço é 0-dimensional (definiremos a seguir), compacto e Hausdorff.

Definição 8.4. Seja $(X, \tau)$ um espaço topológico. Dizemos que $X$ é 0-dimensional se sua topologia possui uma base de conjuntos abertos-fechados.

Proposição 8.5. Seja Ult $(A)$ um espaço de Stone. Temos que Ult(A) é:

(1) 0-dimensional;

(2) Hausdorff;

(3) Compacto.

Demonstração. Seja $\left\{a^{*}: a \in A\right\}$ uma base para a topologia.

(1) Note que $U l t(A) \backslash a^{*}=-\left(a^{*}\right)=(-a)^{*}$. Então cada $a^{*}$ é aberto-fechado.

(2) Sejam $u_{1}, u_{2} \in U l t(X)$ tais que $u_{1} \neq u_{2}$ então existe $a \subset X$ tal que $a \in u_{1}$ e $a \notin u_{2}{ }^{3}$. Como $a \notin u_{2}$, então $-a \in u_{2}$. Assim $u \in a^{*}$ e $u_{2} \in(-a)^{*}$.

Afirmamos que $a^{*} \cap(-a)^{*}=\emptyset$. Suponha que exista $u \in a^{*} \cap(-a)^{*}$, então $a \in u$ e $-a \in u$ e $\operatorname{assim} \emptyset=a \cap(-a) \in u$ o que é um absurdo.

(3) Seja $\mathscr{C}$ uma cobertura aberta de $U l t(X)$. Podemos assumir que $\mathscr{C} \subset\left\{a^{*}: a \subset X\right\}$. Suponha que $\mathscr{C}$ não admita uma subcobertura finita, então dados $a_{1}, \ldots, a_{n} \subset X$ tais que $a_{j}^{*} \in \mathscr{C}$, existe $u \in U l t(X) \backslash\left(a_{1}^{*} \cup \cdots \cup a_{n}^{*}\right)=\left(-a_{1}\right)^{*} \cap \cdots \cap\left(-a_{n}\right)^{*}=$ $\left(-a_{1} \cap \cdots \cap-a_{n}\right)^{*}$.

Ou seja, temos que $\left\{-a: a^{*} \in \mathscr{C}\right\}$ tem p.i.f. então existe $u$ ultrafiltro tal que $-a \in u$ para todo a tal que $a^{*} \in \mathscr{C}$. Assim, $u \in(-a)^{*} \forall a^{*} \in \mathscr{C}$ e, portanto $\mathscr{C}$ não é recobrimento de $U l t(X)$. Absurdo. Portanto $U l t(X)$ é compacto.

\footnotetext{
${ }^{3}$ Pois se para todo $a \subset X$ tal que $a \in u_{1}$ então $a \in u_{2}$ e daí $u_{1} \subset u_{2}$ e portanto $u_{1}$ não seria ultrafiltro.
} 


\subsection{Convergência de ultrafiltros}

Nessa seção definiremos o que é a convergência de ultrafiltros. Veremos algumas equivalências e com isso mostraremos uma aplicação interessante: a demonstração do Teorema de Tychonoff via convergência de ultrafiltros. Lembrando que a álgebra de Boole que trabalharemos nesse capítulo será $(A,+,-, 0,1)=(\wp(X), \cup, \cap, \emptyset, X)$ onde $(X, \tau)$ é um espaço topológico.

Dizemos que $u$ é um filtro sobre $X$ se ele for um filtro na álgebra de Boole $\wp(X)$.

Definição 8.6. Seja $X$ um espaço topológico Hausdorff e $F$ um ultrafiltro sobre $X$. Dizemos que $x \in X$ é um ponto aderente à $F$ se $x \in \bar{Y}$ para todo $Y \in F$. Dizemos que $F$ converge para $x$ (e denotaremos por $F \rightarrow x$ ) se, qualquer vizinhança $V$ de $x$, temos $V \in F$.

Proposição 8.7. Seja X um espaço topológico. São equivalentes:

(1) X é compacto;

(2) toda familia de fechados de X com p.i.f. tem intesecção não vazia;

(3) todo ultrafiltro sobre $X$ tem um ponto aderente;

(4) todo ultrafiltro sobre $X$ é convergente

Demonstração.

$(1) \Rightarrow(2)$ : Feito no Teorema 2.17Noções Básicas de Topologiateorema.2.17.

(2) $\Rightarrow(3)$ : Seja $F$ um ultrafiltro sobre $X$. Considere $A:=\{\bar{Y}: Y \in F\}$. Como $A$ tem a p.i.f., por (2) temos que existe $y \in \bigcap_{Y \in F} \bar{Y}$. Note que $y$ é o ponto aderente a $F$.

$(3) \Rightarrow(4)$ : Seja $F$ um ultrafiltro sobre $X$. Seja $y \in X$ um ponto aderente a $F$. Vamos mostrar que $F \rightarrow y$. Seja $V$ uma vizinhança de $x$. Temos pela Proposição 1.56Filtros e Ultrafiltrosteorema.1.56 que ou $V \in F$ ou $X \backslash V \in F$. Suponha que $X \backslash V \in F$. Como $y$ é ponto aderente a $F$, temos que $y \in \overline{X \backslash V}$. Absurdo, pois $y \in V$ e $V \cap(X \backslash V)=\emptyset$.

$(4) \Rightarrow(1)$ : Seja $\mathcal{C}$ uma cobertura aberta de $X$. Suponha que $\mathcal{C}$ não admite uma subcobertura finita. Temos que $\mathcal{B}=\left\{X \backslash \cup \mathcal{C}^{\prime}: \mathcal{C}^{\prime} \subset \mathcal{C}\right.$ finito $\}$ gera um filtro $F$ sobre $X$, ou seja, $F=\{Y: Y \supset B$ para algum $B \in \mathcal{B}\}$. 
Logo,temos que existe um ultrafiltro $G$ que contém $F$. Por (4) existe $x \in X$ tal que $G \rightarrow x$. Seja $Y \in \mathcal{C}$ tal que $x \in Y$. Considere $\mathcal{C}^{\prime}=\{Y\}$. Note que $X \backslash Y \in G$ e portanto $Y \notin G$ o que contraria o fato que $G \rightarrow x$, pois $Y$ é vizinhança de $x$.

Com tal resultado fica fácil demostrar o Teorema de Tychonoff.

Teorema 8.8 (Teorema de Tychonoff). Seja I um conjunto não vazio. Para cada $i \in I$, seja $X_{i}$, um espaço topológico compacto. Temos que $\prod_{i \in I} X_{i}$ é compacto.

Demonstração. Suponha $X:=\prod_{i \in I} X_{i} \neq \emptyset$. Para cada $i \in I$ considere $\pi_{i}: X \rightarrow X_{i}$ a projeção na $i$-ésima coordenada. Seja $U$ um ultrafiltro sobre $X$. Considere, para cada $i \in I$

$$
U_{i}=\left\{\pi_{i}[A]: A \in U\right\}
$$

Note que $U_{i}$ é um ultrafiltro sobre $X_{i}$. Para cada $i \in I$, seja $a_{i} \in X_{i}$ tal que $U_{i} \rightarrow a_{i}$. Considere $a:=\left\{a_{i}\right\}_{i \in I}$. Vamos mostrar que, dado $V$ aberto básico de $X$ tal que $a \in V$, temos que $V \in U$. Sejam $V:=\prod_{i \in I} V_{i}$ um aberto básico tal que $a \in V$ e $J=\{i \in I$ : $\left.V_{i} \neq X_{i}\right\}$. Suponha $J \neq \emptyset$. Seja $j \in J$. Como $a_{j} \in V_{j}$, temos que $V_{j} \in U_{j}$. Logo, pela definição de $U_{j}$, existe $A \in U$ para cada $j$, tal que $V_{j}=\pi_{j}[A]$. Assim $\pi_{j}^{-1}\left[V_{j}\right] \in U$. Como $V=\bigcap_{j \in J} \pi_{j}^{-1}\left[V_{j}\right] \in U$, o resultado segue.

\subsection{Compactificação de Stone-Cech dos Naturais via es- paços de Stone}

Vamos construir um espaço compacto onde o conjunto dos naturais é denso e que tem a seguinte propriedade: Toda função que sai dos naturais e chega num compacto admite uma extensão contínua para esse espaço. Uma maneira natural de se pensar nisso é analisar se o compactificado de Alexandrov satisfaz as condições impostas.

Consideremos a seguinte situação: Seja $f: \omega \rightarrow[0,1]$ definida como $f(n)=0$ se $n$ é par e $f(n)=1$, se $n$ é impar. Considere o compactificado de Alexandrov de $\omega$ dado por $\omega \cup\{\omega\}$. Suponha que exista $\tilde{f}: \omega \cup\{\omega\} \rightarrow[0,1]$ tal que $\tilde{f}(\omega)=x$, para algum $x \in[0,1]$ e $\tilde{f}(n)=f(n)$ para $n \in \omega$. 
Seja $V$ uma vizinhança de $x$ tal que:

(1) $0,1 \notin V$ se $x \in] 0,1[$, ou

(2) $0 \in V$ e $1 \notin V$ se $x=0$, ou

(3) $0 \notin V$ e $1 \in V$, se $x=1$.

Agora, seja $\tilde{f}^{-1}(V)$ com $\omega \in \tilde{f}^{-1}(V)$. A topologia de $\omega \cup\{\omega\}$ é dada pela topologia de $\omega$ acrescida de elementos da forma $A \cup\{\omega\}$ onde $A^{c}$ é compacto. Note que os subconjuntos compactos em $\omega$ são finitos, então um conjunto aberto que contenha $\omega$ tem complementar finito. Assim, se $B$ é um aberto contendo $\omega$, segue que $B$ contém pontos pares e ímpares e daí temos um absurdo.

Com isso vimos que não conseguimos estender funções contínuas (mantendo a continuidade) usando a extensão de Alexandrov. Vamos "preparar o terreno" para podermos usar o espaço de Stone dos naturais, i.e. $s(\wp(\omega))$ e com isso consiguiremos as extensões.

Proposição 8.9. Seja $f: \omega \rightarrow K$, onde $K$ é compacto e Hausdorff e que $f$ seja limitada. Então dado u ultrafiltro o conjunto $f_{u}=\{A \subset K: \exists a \in u$ tal que $f[a] \subset A\}$ é ultrafiltro na imagem de $f$. Além disso, $\tilde{f}: s(\wp(\omega)) \rightarrow K$ dada por $\tilde{f}[u]=x$ onde $x$ é o ponto para o qual $f_{u}$ converge, é uma extensão contínua de $f$.

Demonstração.

Vamos mostrar que $f_{u}$ é um filtro.

(1) $\emptyset \notin f_{u}$ : De fato, suponha que $\emptyset \in f_{u}$, então existe $a \in u$ tal que $f[a] \subset \emptyset$. Absurdo, pois $\emptyset \notin u$.

(2) Para todo $A \subset f_{u}$ : e todo $B \subset K \operatorname{com} A \subset B$, então $B \in f_{u}$. Como $A \in f_{u}$ então existe $a \in u$ tal que $f[a] \subset A \subset B \operatorname{logo} f[a] \in B$ e, portanto, $B \in f_{u}$.

(3) Dados $A, B \in f_{u}$, então $A \cap B \in f_{u}$ : Sejam $A, B \in f_{u}$. Então existem $a, b \in u$ tais que $f[a] \in A$ e $f[b] \in B$. Note que, por $u$ ser ultrafiltro, segue que $a \cap b \in u$ e $f[a \cap b] \subset f[a] \cap f[b] \subset A \cap B$. Portanto $A \cap B \in f_{u}$. 
Agora, mostremos que $f_{u}$ é ultrafiltro.

Seja $B \subset K$ e suponha que $B \notin f_{u}$. Seja $B^{*}=\{n \in \omega: f(n) \in B\}$. Se $B^{*} \notin u$, então $\omega \backslash B^{*} \in u$. Logo, $f\left[\omega \backslash B^{*}\right]=\bigcup_{n \in\left(\omega \backslash B^{*}\right)} f(n) \subset K \backslash B$. Portanto $K \backslash B \in f_{u}$.

Seja $u_{n}=\{A \subset \omega: n \in A\}$ e observe que $u_{n}$ é um ultrafiltro. De fato:

(1) $\emptyset \notin u_{n}$. Pois não existe $n \in \omega$ tal que $n \in \emptyset$.

(2) Se $a \in u_{n}, b \subset \omega \operatorname{com} a \subset b$ então $b \in u_{n}$. Se $a \in u_{n}$ então $n \in a \subset b \operatorname{logo} n \in b$ e portanto $b \in u_{n}$.

(3) Se $a, b \in u_{n}$ então $a \cap b \in u_{n}$. Sejam $a, b \in u_{n}$ então $n \in a \cap b$ e portanto $a \cap b \in u_{n}$.

(4) $u$ é ulltrafiltro. Seja $a \notin u_{n}$ então $n \notin a$ daí $n \in \omega \backslash a$ implica que $\omega \backslash a \in u_{n}$.

Dizemos que $u_{n}$ é o ultrafiltro principal gerado por $n$.

Mostremos que existe uma cópia de $\omega$ em $s(\wp(\omega))$. Seja $N=\left\{u_{n}: n \in N\right\}$. Observe que:

(1) $N$ é enumerável.

(2) Cada elemento de $N$ é isolado. De fato, seja $u_{n} \in N$ e tome o aberto básico $\{n\}^{*}=$ $\{u:\{n\} \in u\}, \log 0 u_{n} \in\{n\}^{*}$. Agora seja $u$ ultrafiltro com $\{n\} \in u$ e tome $a \in u_{n}$ então $n \in a$ assim $a \in u$ e daí $u_{n} \subset u$ (disso conclui-se que $u_{n}$ é o menor ultrafiltro que contem $\{n\})$. Sejam $m \neq n$ e suponha que $a \in\{m\}^{*} \cap\{n\}^{*}$ então $\{m\},\{n\} \in a$ $\log \emptyset=\{m\} \cap\{n\} \in a$ o que é um absurdo. Portanto cada $\{n\}^{*}$ é unitário e os elementos de $\left\{\{n\}^{*}: n \in \omega\right\}$ dois a dois são disjuntos.

Com isso, podemos construir um homeomorfismo de $\omega$ em $N$.

(3) N é denso em $s(\wp(\omega))$.

Para isso, vamos enunciar o seguinte Lema:

Lema 8.10. Seja $\mathcal{B}$ uma base para a topologia de $X$. Então, $D \subset X$ é denso em $X$ se, e somente se, $D \cap B \neq \emptyset$ para todo $B \in \mathcal{B}$, com $B \neq \emptyset$. 
Demonstração. Se $D$ é denso em $X$ então para todo aberto $U \neq \emptyset$ temos que $U \cap D \neq \emptyset$ e em particular $B \cap D \neq \emptyset$ para $B \in \mathcal{B}$. Reciprocamente, seja $U$ aberto e como $U=\bigcup_{\lambda} B_{\lambda} \operatorname{com} B_{\lambda} \in \mathcal{B}$ então $U \cap D \neq \emptyset$.

Seja $a^{*}=\{u: a \in u\}$ um aberto básico de $s(\wp(\omega))$, temos que $a \subset \omega$ e $a \neq \emptyset$ pois $u$ é ultrafiltro, então tome $k \in a, \log o a \in u_{k}$ e $u_{k} \in a^{*}$. Portanto $N \cap a^{*} \neq \emptyset$ para todo $a^{*}$ aberto básico de $s(\wp(\omega))$. Como consequência do Lema anterior $N$ é denso $\operatorname{em} s(\wp(\omega))$.

Observação 8.11. Chamaremos $s(\wp(\omega))$ de $\beta \omega$.

Continuação da demonstração da Proposição 8.9Compactificação de Stone-Cech dos Naturais via espaç

Mostremos que $\tilde{f}\left(u_{n}\right)=f(n)$. Seja $x=\tilde{f}\left(u_{n}\right)$, ou seja, $x$ é o limite de $f_{u_{n}}$ e suponha que $f(n) \neq x$, então existe um aberto $V$ tal que $x \in V$ e $f(n) \notin V$, pela definição de convergência de ultrafiltros segue que $V \in f_{u_{n}}$. Por outro lado $f(n) \in f_{u_{n}}$, pois $\{n\} \in u_{n}$ e daí $f[\{n\}] \in\{f(n)\}$. Assim, $\emptyset=V \cap f[n] \in f_{u_{n}}$ o que é um absurdo. Portanto $\tilde{f}\left(u_{n}\right)=f(n)$, em outras palavras, $\tilde{f}$ estende $f$.

Observe que $\tilde{f}$ é contínua. Seja $U \subset K$ um aberto. Tome $u \in \tilde{f}^{-1}[U]$, como $K$ é regular então existe um aberto $V \subset K$ tal que $\tilde{f}(u) \in V \subset \bar{V} \subset U$. Então $V \in f_{u}$, ou seja, existe $a \in u$ tal que $f[a] \subset V \subset \bar{V}$ e então $u \in \tilde{f}^{-1}[V] \subset \tilde{f}^{-1}[\bar{V}]$.

Mostremos que $a^{*} \subset \tilde{f}^{-1}[\bar{V}]$. Seja $b \in a^{*}$ e suponha que $\tilde{f}(b) \notin \bar{V}$ então $\tilde{f}(b) \in K \backslash \bar{V}$ que é um aberto, então $K \backslash \bar{V} \in f_{b}$. Por outro lado, como $b \in a^{*}$ que implica $a \in b$ e também $f[a] \subset \bar{V}$ implica que $\bar{V} \in f_{b}$. Logo $\emptyset=(K \backslash \bar{V}) \cap \bar{V} \in f_{b}$ o que é uma contradição. Portanto $a^{*} \subset \tilde{f}^{-1}[\bar{V}] \subset \tilde{f}^{-1}[U], \operatorname{logo} \tilde{f}^{-1}[U]$ é aberto e portanto $\tilde{f}$ é contínua.

Para finalizar a proposição, veja que essa extensão é única a menos de homeomorfismos.

Lema 8.12. Sejam $A$ e $B$ espaços topológicos e $B$ de Hausdorff. Considere $D \subset A$ denso em $A$ e sejam $f, g: A \rightarrow B$ continuas. Se $f(x)=g(x)$ para todo $x \in D$ então $f=g$.

Demonstração. Suponha que exista $y \in A$ tal que $f(y) \neq g(y)$. Como $B$ é Hausdorff então existem $U$ e $V$ abertos tal que $f(y) \in U$ e $g(y) \in V \operatorname{com} U \cap V \neq \emptyset$. Como $f$ e $g$ são contínuas então $f^{-1}(U)$ e $g^{-1}(V)$ são abertos e $f^{-1}(U) \cap g^{-1}(V)$ é um aberto 
não vazio, pois $y \in f^{-1}(U) \cap g^{-1}(V)$. Como D é denso em A, então existe $d \in D$ com $d \in f^{-1}(U) \cap g^{-1}(V)$ e daí $f(d) \neq g(d)$ o que é um absurdo, pois $\left.f\right|_{D}=\left.g\right|_{D}$. Portanto $f=g$.

Agora, suponha que exista outro espaço compacto e Hausdorff $Y$ tal que $\omega \subset Y$ com $\bar{\omega}=Y$ e que $Y$ tem a propriedade da extensão de funções contínuas (p.e.f.c.) de $\omega$. Seja $i d: \omega \rightarrow Y$ a função identidade. Como $\beta \omega$ tem p.e.f.c. de $\omega$ segue que existe $f: \beta \omega \rightarrow Y$ contínua. Considere também $i d: \omega \rightarrow \beta \omega$ a função identidade. Como $Y$ tem p.e.f.c. de $\omega$ segue que existe $g: Y \rightarrow \beta \omega$ contínua. Logo

$$
\left.f \circ g\right|_{\omega}=\left.g \circ f\right|_{\omega}=i d
$$

Como $\bar{\omega}=\beta \omega$ e $\bar{\omega}=Y$, pelo Lema anterior segue que $\beta \omega$ é homeomorfo a $Y$.

Depois de todo esse trabalho, o espaço desejado para a extensão é $\beta \omega$ e agora veremos uma aplicação desse espaço à análise funcional.

Proposição 8.13. $\ell_{\infty}{ }^{4}$ é isomorfo ${ }^{5}{ }^{a} \mathcal{C}(\beta \omega)^{6}$.

Demonstração. Considere

$$
\begin{aligned}
\varphi: \mathcal{C}(\beta \omega) & \rightarrow \ell_{\infty} \\
\tilde{f} & \mapsto \varphi(\tilde{f})=(f(n))_{n \in \omega}
\end{aligned}
$$

onde $\left.\tilde{f}\right|_{\omega}=f$.

Observe que $\|\tilde{f}\|_{\mathcal{C}(\beta \omega)}=\sup _{x \in \mathcal{C}(\beta \omega)}\|\tilde{f}(x)\|<\infty$ e daí $\sup _{n \in \omega}\|\tilde{f}(n)\|<\infty$. Portanto $\varphi$ está bem definida.

Note que $\varphi$ é linear.

Sejam $\lambda \in \mathbb{R}$ e $\tilde{f} \in \mathcal{C}(\beta \omega)$. Logo $\varphi(\lambda \tilde{f})=(\lambda f(n))_{n \in \omega}=\lambda \varphi(\tilde{f})$.

\footnotetext{
${ }^{4} \ell_{\infty}$ é o espaço das sequências $\left(a_{n}\right)_{n \in \omega}$ onde $a_{n} \in \mathbb{R}$ com $\sup _{n} a_{n}$ finito. Além disso, se $x \in \ell_{\infty}$ então $x=\left(a_{n}\right)_{n \in \omega}$ e $\|x\|=\sup _{n} a_{n}$.

${ }^{5}$ Como espaço de Banach

${ }^{6}$ Espaço das funções contínuas de $\beta \omega$ em $\mathbb{R}$.
} 
$\operatorname{Sejam} \tilde{f}, \tilde{g} \in \mathcal{C}(\beta \omega)$. Então $\varphi(\tilde{f}+\tilde{g})=((f+g)(n))_{n \in \omega}=(f(n))_{n \in \omega}+(g(n))_{n \in \omega}=$ $\varphi(\tilde{f})+\varphi(\tilde{g})$.

- $\varphi$ é sobrejetora.

Seja $x=\left(x_{n}\right) \in \ell_{\infty}$, assim existe uma função $f: \omega \rightarrow \mathbb{R}$ limitada tal que $f(n)=x_{n}$ e como $\beta \omega$ possui a propriedade da extensão de funções contínuas de $\omega$ segue que existe $\tilde{f} \in \mathcal{C}(\beta \omega)$ tal que $\left.\tilde{f}\right|_{\omega}=f$. Portanto $\varphi(\tilde{f})=(f(n))_{n}=\left(x_{n}\right)=x$.

- $\varphi$ é injetora.

Sejam $\tilde{f}, \tilde{g}: \beta \omega \rightarrow \mathbb{R}$ e $\omega \subset \beta \omega$ com $\bar{\omega}=\beta \omega$ e $\tilde{f} \neq \tilde{g}$ então pela contrapositiva do Lema 8.12Compactificação de Stone-Cech dos Naturais via espaços de Stoneteorema.8.12 segue que $\left.\tilde{f}\right|_{\omega} \neq\left.\tilde{g}\right|_{\omega}$.

- $\varphi$ é uma isometria.

(1) $\|\varphi(\tilde{f})\|_{\infty}=\left\|(f(n))_{n}\right\| \leq\|\tilde{f}\|_{\mathcal{C}(\beta \omega)}$.

(2) Dado $\varepsilon>0$ existe $x \in \beta \omega$ tal que

$$
\|\tilde{f}\|_{\mathcal{C}(\beta \omega)}-\varepsilon<|\tilde{f}(x)| \leq\|\tilde{f}\|_{\mathcal{C}(\beta \omega)}
$$

Como $\tilde{f}$ é uniformemente contínua tome $\delta$ de forma que para todo $x, y \in \beta \omega$ $\operatorname{com} \operatorname{dist}(x, y)<\delta$ temos que $\|\tilde{f}(x)-\tilde{f}(y)\|<\varepsilon \operatorname{com} \varepsilon$ dado acima. Como $\omega$ é denso em $\beta \omega$ então para esse mesmo $\delta>0$ e $x \in \beta \omega$ existe $x_{n} \in \omega$ tal que $\left|x-x_{n}\right|<\delta$ então $\left|\tilde{f}(x)-f\left(x_{n}\right)\right|<\varepsilon \Rightarrow|\tilde{f}(x)|<\left|f\left(x_{n}\right)\right|+\varepsilon$. Logo

$$
\|\left.\tilde{f}\right|_{\mathcal{C}(\beta \omega)}-\varepsilon<|\tilde{f}(x)|<\left|f\left(x_{n}\right)\right|+\varepsilon
$$

Como $\varepsilon$ é arbitrário então

$$
\|\tilde{f}\|_{\mathcal{C}(\beta \omega)} \leq \sup _{n \in \omega}|f(n)|=\|\varphi(\tilde{f})\|_{\infty}
$$




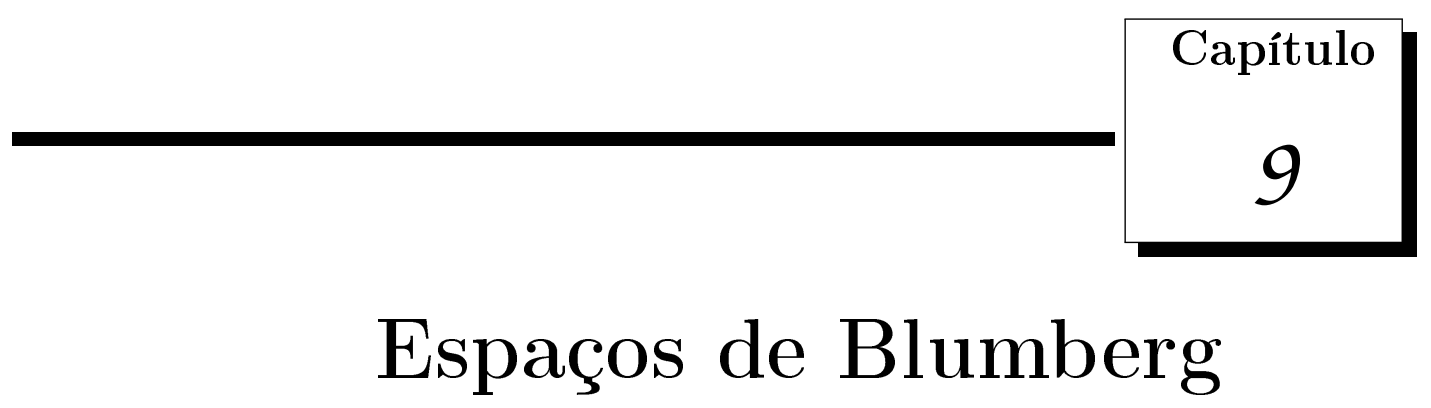

Nesse capítulo veremos quais condições um espaço topológico $X$ tem que satisfazer para que dada uma função à valores reais exista um subconjunto denso $D$ tal que a restrição da função à $D$ seja contínua. Os espaços que possuem essa propriedade chamaremos de espaços de Blumberg.

\subsection{Espaços de Blumberg}

Dada uma função como saber se ela é contínua? Sabemos que se duas funções contínuas coincidem em um subconjunto denso elas são iguais no todo. Podemos nos perguntar: se ela for contínua num subconjunto denso ela é contínua no espaço todo?

Isso não é verdade. Por exemplo, tomando-se a função característica dos racionais observamos que sua restrição aos racionais é contínua mas a função está longe de ser contínua. De fato, é descontínua em toda a reta.

Um resultado "surpreendente" que Henri Blumberg provou em 1922 é que para funções da reta na reta, em geral, temos que existe um subconjunto denso cuja restrição da função é contínua, ou seja qualquer função não contínua serve de contra-exemplo. Isso inspira a seguinte definição:

Definição 9.1. Seja $X$ um espaço topológico. Se para toda função $f: X \rightarrow \mathbb{R}$ existir um subconjunto denso $D \subset X$ tal que $f \mid D$ é continua em $D$ dizemos que o espaço $X$ é de 


\section{Blumberg.}

Em 1960 J.C. Bradford e C. Goffman generalizaram tal resultado provando: Um espaço métrico é Blumberg se, e somente se, é um espaço de Baire.

Nessa seção inicialmente mostraremos que se $X$ é um espaço de Blumberg então é de Baire. Posteriormente enunciaremos alguns resultados que serão necessários para demonstrar o resultado enunciado acima de maneira um pouco mais generalizada.

Definição 9.2. Seja $X$ um espaço topológico. Dado $A \subset X$, dizemos que $A$ é nunca denso em $X$ se o interior de $(\bar{A})$ é $\emptyset$. Um subconjunto de $X$ é dito ser de $\mathbf{1}^{\boldsymbol{a}}$ categoria em $X$ se é composto de uma união enumerável de conjuntos nunca densos. Um subconjunto de $X$ é dito ser de $2^{a}$ categoria se não é de $1^{a}$ categoria.

Teorema 9.3. Se X é um espaço de Blumberg então X é um espaço de Baire.

Demonstração. Suponha que $X$ não seja de Baire. Então existe uma sequência $\left(A_{n}\right)_{n \in \omega}$ de abertos densos tais que $\bigcap_{n \in \omega} A_{n}$ não é densa em $X$, isto é, existe um aberto não vazio $U \subset X$ tal que $U \cap\left(\bigcap_{n \in \omega} A_{n}\right)=\emptyset$. Equivalentemente, existe uma família enumerável de fechados $\left(F_{n}\right)_{n \in \omega} \operatorname{com} \operatorname{int}\left(F_{n}\right)=\emptyset$ tal que $\operatorname{int}\left(\bigcup_{n \in \omega} F_{n}\right) \neq \emptyset$. Defina a seguinte função $f: X \rightarrow \mathbb{R}:$

$$
f(x)= \begin{cases}n & \text { se } n=\min \left\{k+1 \in \omega: x \in F_{k}\right\} \\ 0 & \text { se } x \notin F\end{cases}
$$

onde $F=\bigcup_{n \in \omega} F_{n}$.

Seja $D \subset X$ um subconjunto denso qualquer de $X$ e seja $x \in D \cap F^{1}$. Então existe um menor índice $n$ tal que $x \in D \cap F_{n}$. Seja $V$ um aberto contendo $x$. Como $\operatorname{int}\left(F_{n}\right)=\emptyset$, existe $x^{\prime} \in\left(V \backslash F_{n}\right) \cap D$. Como $\left|f(x)-f\left(x^{\prime}\right)\right| \geq 1$, temos que $f \mid D$ não é contínua em $x \in D$.

A recíproca, em geral, não é verdadeira, na próxima seção construiremos um espaço que ilustra isso. Além disso na referência [6] temos contra-exemplos de Levy e White.

Agora veremos alguns resultados auxiliares para demonstrar o Teorema 9.10Espaços de Blumbergteorema.9.10. Que consiste em mostrar que: Dado $X$ um espaço métrico e

\footnotetext{
${ }^{1}$ Existe $x \in D \cap F$, pois $D$ é denso e $\operatorname{int}(F) \neq \emptyset$.
} 
de Baire e $Y$ um espaço topológico com base enumerável e uma função $f: X \rightarrow Y$. Então existe um subespaço denso $D \subset X$ tal que $f \mid D$ é contínua em $D$.

Definição 9.4. Seja $X$ um espaço topológico. Uma família $\mathcal{N}$ de subconjuntos de $X$ é localmente finita em $A \subset X$ se para cada $x \in A$ existe uma vizinhança de $x$ que intercepta apenas uma quantidade finita de elementos de $\mathcal{N}$.

Lema 9.5. Seja $X$ um espaço topológico. Seja $\mathcal{N}$ uma familia de subconjuntos nunca densos de $X$. Se $\mathcal{N}$ é localmente finita num subconjunto denso de $X$, então $\bigcup_{N \in \mathcal{N}} N$ é nunca denso em $X$.

Demonstração. Seja $\mathcal{N}=\left\{N_{\alpha}: \alpha \in A\right\}$ uma família localmente finita em um subconjunto denso $D \subset X$ onde cada $N_{\alpha}$ é um subconjunto nunca denso em $X$.

Note que $\left\{\overline{N_{\alpha}}: \alpha \in A\right\}$ também é localmente finito em $D$, pois para cada $x \in D$ existe $V_{x} \ni x$ aberto tal que $V_{x}$ intercepta apenas uma quantidade finita de elementos de $\mathcal{N}$, digamos $\left\{N_{\alpha_{j}}: j=1, \ldots, m\right\}$, então $V_{x}$ intercepta apenas $\left\{\overline{N_{\alpha_{j}}}: j=1, \ldots, m\right\}$.

Seja $U$ um aberto arbitrário não vazio de $X$. Assim existe um subconjunto não vazio aberto $V \subset U$ que intercepta uma quantidade finita de elementos de $\left\{\overline{N_{\alpha}}: \alpha \in A\right\}^{2}$, digamos $\left\{\overline{N_{\alpha_{i}}}: i=1,2, \ldots, n\right\}$. Como cada $X \backslash \overline{N_{\alpha_{i}}}$ é um subconjunto aberto denso de $X$, então $W=V \cap\left[\bigcap_{i=1}^{n}\left(X \backslash \overline{N_{\alpha_{i}}}\right)\right] \neq \emptyset$. A última implicação decorre da seguinte afirmação:

Se $A_{1}, A_{2}, \ldots, A_{n}$ são abertos densos em $X$, então $\bigcap_{i=1}^{n} A_{i}$ é aberto denso em $X$. Tome um aberto não vazio $O \subset X$ e observe que $O \cap A_{1}$ é aberto não vazio e portanto $O \cap\left(A_{1} \cap A_{2}\right) \neq \emptyset$.

Pela escolha de $V$ temos que $W \subset \bigcap_{a \in A}\left(X \backslash \overline{N_{\alpha}}\right)=X \backslash\left(\bigcup_{a \in A} \overline{N_{\alpha}}\right)$, pois $V \subset\left(X \backslash \overline{N_{\alpha}}\right)$ para $\alpha \neq \alpha_{i}$. Portanto, $U$ intercepta $X \backslash\left(\bigcup_{a \in A} \overline{N_{\alpha}}\right)$. Logo por $U$ ser arbitrário segue que $\bigcup_{N \in \mathcal{N}} N$ é nunca denso em $X$.

Lema 9.6. Seja X um espaço topológico. A união de qualquer familia de abertos de $1^{a}$ categoria é de $1^{a}$ categoria.

Demonstração. Seja $\mathcal{U}$ uma família de abertos não vazios de $1^{\text {a }}$ categoria. Seja $\mathcal{V}=$ $\left\{V_{\alpha}: \alpha \in A\right\}$ uma família maximal de abertos não vazios dois a dois disjuntos com a propriedade de que cada elemento de $\mathcal{V}$ está contido em algum elemento de $\mathcal{U}$.

\footnotetext{
${ }^{2}$ Como $D$ é denso em $X$ existe $x \in U \cap D$.
} 
Portanto, $\overline{\bigcup_{U \in \mathcal{U}} U} \backslash \bigcup_{\alpha \in A} V_{\alpha}$ é nunca denso em $X$. Suponha que não, então existe um aberto não vazio $B$ onde $B \subset \overline{\bigcup_{U \in \mathcal{U}} U} \backslash \bigcup_{\alpha \in A} V_{\alpha}$. Então $B$ intercepta $U^{\prime}$ para algum $U^{\prime} \in \mathcal{U}$, logo $B \cap U^{\prime}$ é aberto não vazio e disjunto dos demais elementos de $\mathcal{V}$ o que é um absurdo.

Para cada $\alpha \in A, V_{\alpha}$ pode ser representado como uma união enumerável de conjuntos nunca densos, digamos, $V_{\alpha}=\bigcup_{i \in \omega} N_{\alpha, i}$. Para cada $i$, seja $N_{i}=\bigcup_{\alpha \in A} N_{\alpha, i}$. Observe que $N_{i}$ é nunca denso em $X$ pelo lema anterior. Para ver isso, seja $x \in \mathcal{V}$, então $x \in V_{\alpha}$ para algum $\alpha \in A$, como cada $V_{\alpha}$ contém exatamente um elemento de cada $N_{i}$, disso segue que $V_{\alpha}$ intercepta um elemento de $N_{i}$. Portanto $N_{i}$ é localmente finito em $\mathcal{V}$ e esse por sua vez é denso em $\bigcup_{U \in \mathcal{U}} U$. Pelo lema anterior $N_{i}$ é nunca denso em $\bigcup_{U \in \mathcal{U}} U$, mas por esse último ser aberto em $X$ segue que $N_{i}$ é nunca denso em $X$.

Portanto,

$$
\bigcup_{U \in \mathcal{U}} U \subset\left[\overline{\bigcup_{U \in \mathcal{U}} U} \backslash \bigcup_{\alpha \in A} V_{\alpha}\right] \cup\left(\bigcup_{\alpha \in A} V_{\alpha}\right)=\left[\overline{\bigcup_{U \in \mathcal{U}} U} \backslash \bigcup_{\alpha \in A} V_{\alpha}\right] \cup\left(\bigcup_{i \in \omega} N_{i}\right)
$$

e $\operatorname{assim} \bigcup_{U \in \mathcal{U}} U$ é de primeira categoria em $X$.

Lema 9.7. Seja $X$ um espaço topológico e seja $A \subset X$. Suponha que para cada aberto não vazio $U$, existe um aberto não vazio $W \subset U$ tal que $W \cap A$ é de $1^{\circ}$ categoria em $X$. Então A é de $1^{o}$ categoria em $X$.

Demonstração. Se $A$ é nunca denso em $X$ então não há nada para se mostrar.

Agora, suponha que $U=\operatorname{int}(\bar{A}) \neq \emptyset$. Seja $\left\{U_{\beta}: \beta \in B\right\}$ a família de todos os subconjuntos abertos não vazios de $X$ contidos em $U$ cuja intersecção com $A$ é de $1^{\circ}$ categoria em $X$. Assim, para cada $\beta \in B, U_{\beta} \cap A$ é de $1^{\circ}$ categoria em $U$, logo, de $1^{\circ}$ categoria em $\bar{A}$ e portanto de $1^{\circ}$ categoria em $A$. Pelo lema anterior segue que $\bigcup_{\beta \in B}\left(U_{\beta} \cap\right.$ $A)$ é de $1^{\circ}$ categoria em $A$ e, portanto, de $1^{\circ}$ categoria em $X$.

Note que $\bigcup_{\beta \in B}\left(U_{\beta} \cap A\right)$ é um aberto denso de $A \cap U$. Pois para cada aberto não vazio $V \subset A \cap U$, pelas hipóteses do lema, existe $\beta \in B$ tal que $\left(U_{\beta} \cap A\right) \subset V$. Então segue que $(A \cap U) \backslash \bigcup_{\beta \in B}\left(U_{\beta} \cap A\right)$ é nunca denso em $A \cap U$ e desse modo nunca denso em $X$. 
Observe que $A \cap U=\left[(A \cap U) \backslash \bigcup_{\beta \in B}\left(U_{\beta} \cap A\right)\right] \cup\left[\bigcup_{\beta \in B}\left(U_{\beta} \cap A\right)\right]$ e assim $A \cap U$ é de $1^{\circ}$ categoria em $X$.

Como $A \backslash U$ é nunca denso em $X$ implica que $A=(A \backslash U) \cup(A \cap U)$ é um conjunto de $1^{\circ}$ categoria em $X$.

Definição 9.8. Seja $X$ um espaço métrico. Seja $A \subset X$ e $x \in X$, então dizemos que $x$ é um ponto relativamente forte (r.f.) com $A$ se existe um $\varepsilon>0$ tal que para todo $z \in B_{\varepsilon}(x)$ e $\delta>0, A \cap B_{\delta}(z)$ é não vazio e de $\mathscr{2}^{\circ}$ categoria em $X$.

Proposição 9.9. Seja $X$ um espaço métrico. Seja $E \subset X$ defina $S(E)$ como $x \in X$ tal que para $\varepsilon>0$ arbitrário $B_{\varepsilon}(x) \cap E$ é não vazio e de $2^{\circ}$ categoria em $X$. Defina $H(E)$ o conjunto dos pontos de $X$ que são r.f. com E. Então:

- $S(E)$ é fechado e está contido em $\bar{E}$;

- $H(E)=\operatorname{int}(S(E))$ e consequêntemente $S(E) \backslash H(E)$ é nunca denso;

- se $E_{1} \subset E_{2} \subset X$, então $S\left(E_{1}\right) \subset S\left(E_{2}\right)$ e $H\left(E_{1}\right) \subset H\left(E_{2}\right)$.

Demonstração. Seja $x \in \overline{S(E)}$ e seja $B_{\varepsilon}(x)$ então para $\varepsilon>0$ arbitrário $B_{\varepsilon}(x) \cap S(E) \neq \emptyset$, $\operatorname{logo}$ existe $y \in B_{\varepsilon}(x) \cap S(E)$. Tome $r_{\varepsilon}>0$ tal que $B_{r_{\varepsilon}}(y) \subset B_{\varepsilon}(x)$. Como $B_{r_{\varepsilon}}(y) \cap E$ é de $2^{\circ}$ categoria em $X$ segue que $B_{\varepsilon}(x) \cap E$ também o é. Portanto $\overline{S(E)} \subset S(E)$ e assim $S(E)$ é fechado. Agora, seja $z \in S(E)$ e seja $B_{\varepsilon}(z)$ para $\varepsilon>0$ arbitrário, como $B_{\varepsilon}(z) \cap E \neq \emptyset$ segue que $z \in \bar{E}$.

Seja $x \in H(E)$, então existe um $r>0$ tal que para todo $z \in B_{r}(x)$ e $\delta>0, E \cap B_{\delta}(z)$ é não vazio e de $2^{\circ}$ categoria em $X$ e então $B_{r}(x) \subset S(E)$ e assim $x \in \operatorname{int}(S(E))$. Seja $y \in \operatorname{int}(S(E))$ então existe $r>0$ tal que $B_{r}(y) \subset S(E)$, logo $y \in H(E)$. Portanto $H(E)=\operatorname{int}(S(E))$.

Seja $E_{1} \subset E_{2} \subset X$. Seja $x \in S\left(E_{1}\right)$, para $\varepsilon>0$ arbitrário temos que $B_{\varepsilon}(z) \cap E_{1}$ é de $2^{\circ}$ categoria em $X$, mas, $B_{\varepsilon}(z) \cap E_{1} \subset E_{1} \subset E_{2}$. Logo $x \in S\left(E_{2}\right)$ e portanto $S\left(E_{1}\right) \subset S\left(E_{2}\right)$. Consequêntemente temos que $\operatorname{int}\left(S\left(E_{1}\right)\right) \subset \operatorname{int}\left(S\left(E_{2}\right)\right)$ que implica $H\left(E_{1}\right) \subset H\left(E_{2}\right)$.

Teorema 9.10. Seja $X$ um espaço métrico e de Baire, seja $Y$ um espaço topológico com base enumerável e seja $f: X \rightarrow Y$. Então existe um subespaço denso $D \subset X$ tal que $f \mid D$ é contínua em D. 
Demonstração. Para cada $x \in X$ e $\varepsilon>0$ tome a bola aberta $B_{\varepsilon}(x)$. Seja $\left\{B_{\varepsilon}(x): x \in X\right.$ e $\varepsilon>0\}$ uma base para a topologia de $X$.

Seja $Z=\{x \in X$ : para todo $G$ aberto não vazio com $f(x) \in G$ temos que $x$ é r.f. com $\left.f^{-1}[G]\right\}$. Nossos esforços nessa parte inicial são para mostrar que $Z$ é denso em $X$.

Seja $\left\{G_{n}\right\}_{n \in \omega}$ uma base enumerável para $Y$, e $H_{n}=\left\{x \in f^{-1}\left[G_{n}\right]: x\right.$ não é r.f. com $\left.f^{-1}\left[G_{n}\right]\right\}$. Para ver que $H_{n}$ é de $1^{\circ}$ categoria em $X$ seja

$A_{n}=\left\{x \in f^{-1}\left[G_{n}\right]\right.$ : existe $\varepsilon>0$ tal que $B_{\varepsilon}(x) \cap f^{-1}\left[G_{n}\right]$ é de $1^{\circ}$ categoria em $\left.X\right\}$.

Pelo Lema 9.7Espaços de Blumbergteorema.9.7, $A_{n}$ é de $1^{\circ}$ categoria em $X$. Seja $B_{n}=\left\{x \in f^{-1}\left[G_{n}\right] \backslash A_{n}: x\right.$ não é r.f. com $\left.f^{-1}\left[G_{n}\right]\right\}$. Logo, pela Proposição 9.9Espaços de Blumbergteorema.9.9 $B_{n}$ é nunca denso em $X$, pois $B_{n}=S\left(f^{-1}\left[G_{n}\right]\right) \backslash H\left(f^{-1}\left[G_{n}\right]\right)$. Portanto $H_{n}$ é de $1^{\circ}$ categoria, pois, $H_{n} \subset A_{n} \cup B_{n}$.

Portanto, $X \backslash Z$ é de $1^{\circ}$ categoria em $X$ pois $X \backslash Z \subset \bigcup_{n \in \omega} H_{n}$. Como $X$ é de Baire $Z$ é denso em $X$.

Esse conjunto denso ainda não é bom o suficiente para o nosso propósito. Nessa próxima etapa vamos refiná-lo sem perder a densidade.

Para cada $y \in Y$, seja $\left\{G_{n}(y)\right\}_{n \in \omega}$ um sistema fundamental de vizinhanças em $y$ com $G_{n+1}(y) \subset G_{n}(y)$ para cada $n$. Também, para cada $z \in Z$ e $n<\omega$, seja $W_{n}(z)=$ $f^{-1}\left[G_{n}(f(z))\right]$. Então existe uma coleção maximal dois a dois disjunta $\mathcal{U}_{1}$ de bolas $B_{\varepsilon_{1}(z)}(z)$ onde vale:

(a) $z \in Z$,

(b) $\varepsilon_{1}(z)<1 / 2$,

(c) se $x \in B_{\varepsilon_{1}(z)}(z)$ e $\delta>0$, então $B_{\delta}(x) \cap W_{1}(z)$ é de $2^{\circ}$ categoria em $X$.

Para ver isso tome $\left\{z_{\gamma}: \gamma<\xi\right\}$ uma boa ordenação de $Z$. Para cada $\gamma$ tentamos construir um raio $\varepsilon_{1}\left(z_{\gamma}\right)<1 / 2$ de modo que $B_{\varepsilon_{1}\left(z_{\gamma}\right)}\left(z_{\gamma}\right)$ satisfaça as propriedades acima. As bolas que foram possíveis construir estão em $\mathcal{U}_{1}$. Se $\mathcal{U}_{1}$ não é maximal, então existe um $z^{\prime} \in Z$ tal que $B_{\varepsilon_{1}\left(z^{\prime}\right)}\left(z^{\prime}\right)$ satisfaz as propriedades acima mas não pertence a $\mathcal{U}_{1}$. Porém existe $\eta<\xi$ tal que $z^{\prime}=z_{\eta}$ então na $\eta$-ésima etapa da construção feita anteriormente a bola $B_{\varepsilon_{1}\left(z^{\prime}\right)}\left(z^{\prime}\right)$ está inclusa em $\mathcal{U}_{1}$. 
Seja $Z_{1}=\left\{z: B_{\varepsilon_{1}(z)}(z) \in \mathcal{U}_{1}\right\}$ e para cada $z \in Z_{1}$, seja $V_{1}(z)=W_{1}(z) \cap B_{\varepsilon_{1}(z)}(z) \cap Z$. Seja $x \in B_{\varepsilon_{1}(z)}(z), B$ uma vizinhança qualquer de $x$ contida em $B_{\varepsilon_{1}(z)}(z)$, então $B \cap W_{1}(z)$ é de $2^{\circ}$ categoria em $X$. Por $X \backslash Z$ ser de $1^{\circ}$ categoria em $X(Z$ ser denso em $X), B \cap V_{1}(z)$ é de $2^{\circ}$ categoria em $X$.

Portanto $V_{1}(z)$ é denso em $B_{\varepsilon_{1}(z)}(z)$. Seja $\mathcal{V}_{1}=\left\{V_{1}(z): z \in Z_{1}\right\}$.

Para construir $\mathcal{U}_{2}$ tomamos pontos em $\left(\bigcup_{z \in Z_{1}} B_{\varepsilon_{1}(z)}(z)\right) \cap Z$ e constuímos bolas $B_{\varepsilon_{2}(z)}(z)$ onde vale:

(a) $\varepsilon_{2}(z)<\min \left\{\varepsilon_{1}(z), 1 / 4\right\}$,

(b) se $x \in B_{\varepsilon_{2}(z)}(z)$ e $\delta>0$, então $B_{\delta}(x) \cap W_{2}(z)$ é de $2^{\circ}$ categoria em $X$.

Seja $Z_{2}=\left\{z: B_{\varepsilon_{2}(z)}(z) \in \mathcal{U}_{2}\right\}$ e para cada $z \in Z_{2}$, seja $V_{2}(z)=W_{2} \cap B_{\varepsilon_{2}(z)}(z) \cap Z$. De maneira análoga $V_{2}(z)$ é denso em $B_{\varepsilon_{2}(z)}(z)$ e seja $\mathcal{V}_{2}=\left\{V_{2}(z): z \in Z_{2}\right\}$.

Note que $Z_{1}, Z_{2} \subset Z$ e $Z_{2} \subset Z_{1}$. Para ver isso seja $z \in Z_{2}$ então $B_{\varepsilon_{2}(z)}(z) \in \mathcal{U}_{2}$ e $\varepsilon_{2}(z)<1 / 2$. Se $x \in B_{\varepsilon_{2}(z)}(z) \in \mathcal{U}_{2}$ e $\delta>0$ então $B_{\delta}(x) \cap W_{2}(z)$ é de $2^{\circ}$ categoria em $X$, como $G_{n+1}(y) \subset G_{n}(y)$ para cada $n$ então $W_{2}=f^{-1}\left[G_{2}\right] \subset f^{-1}\left[G_{1}\right]=W_{1}$ e assim $B_{\delta}(x) \cap W_{1}(z)$ é de $2^{\circ}$ categoria em $X$.

Além disso $\bigcup_{z \in Z_{1}} B_{\varepsilon_{1}(z)}(z)$ e $\bigcup_{z \in Z_{2}} B_{\varepsilon_{2}(z)}(z)$ são densos em $X$ pela maximalidade das bolas.

Procedendo por indução suponha que as sequências finitas $\left\{Z_{n}: n=1, \ldots, k\right\},\left\{\varepsilon_{n}\right.$ : $n=1, \ldots, k\},\left\{\mathcal{U}_{n}: n=1, \ldots, k\right\}$ e $\left\{\mathcal{V}_{n}: n=1, \ldots, k\right\}$ estejam definidos e que as seguintes afirmações sejam verdadeiras para cada $n=1, \ldots, k$ satisfazendo:

(i) $Z_{n} \subset Z$.

(ii) $Z_{n+1} \subset Z_{n}$.

(iii) $\varepsilon_{n}: Z_{n} \rightarrow(0,+\infty)$ função.

(iv) Para cada $z \in Z_{n}, \varepsilon_{n}<1 / 2 n$.

(v) $\mathcal{U}_{n}=\left\{B_{\varepsilon_{1}(z)}(z): z \in Z_{n}\right\}$

(vi) Os elementos de $\mathcal{U}_{n}$ são dois a dois disjuntos. 
(vii) $\bigcup_{z \in Z_{n}} B_{\varepsilon_{n}(z)}(z)$ é densa em $X$.

(viii) $\mathcal{V}_{n}=\left\{V_{n}(z): z \in Z_{n}\right\}$ tal que para todo $z \in Z_{n} z \in V_{n}(z) \subset B_{\varepsilon_{n}(z)}(z) \cap W_{n}(z)$.

(ix) Para cada $z \in Z_{n}, V_{n}(z)$ é denso em $B_{\varepsilon_{n}(z)}(z)$.

Para construir o passo $n+1$ basta repetir o que foi feito no passo 2 e portanto definimos indutivamente sequências $\left\{Z_{n}: n \in \omega\right\},\left\{\varepsilon_{n}: n \in \omega\right\},\left\{\mathcal{U}_{n}: n \in \omega\right\}$ e $\left\{\mathcal{V}_{n}: n \in \omega\right\}$ tal que para cada $n$ valham as propriedades de $(i)$ a $(i x)$ listadas acima.

Seja $D=\bigcup_{n \in \omega} Z_{n}$. Observe que $D$ é denso em $\bigcap_{n \in \omega} \bigcup_{B \in \mathcal{U}_{n}} B$. Suponha que não então existe um aberto não vazio $V \subset \bigcap_{n \in \omega} \bigcup_{B \in \mathcal{U}_{n}} B$ cuja intersecção com $D$ é vazia. Mas $V=A \cap\left(\bigcap_{n \in \omega} \bigcup_{B \in \mathcal{U}_{n}} B\right)$ para $A \subset X$ aberto, então $V=\bigcap_{n \in \omega} \bigcup_{B \in \mathcal{U}_{n}}(B \cap A)$ e para cada $B \cap A$ conseguimos $B^{\prime} \in \mathcal{U}_{n}$ tal que $B^{\prime} \subset B \cap A$. Seja $V^{\prime}=\bigcap_{n \in \omega} \bigcup_{B \in \mathcal{U}_{n}} B^{\prime}$ então $V^{\prime} \subset V$ e $V^{\prime} \cap D=\emptyset$. Isso significa que existem centros de bolas $z$ de $B_{\varepsilon_{k}(z)}(z) \in \mathcal{U}_{k}$ para algum $k$ que não estão em $V^{\prime}$ o que é um absurdo.

Por $X$ ser de Baire segue que $\bigcap_{n \in \omega} \cup_{B \in \mathcal{U}_{n}} B$ é denso em $X$ e portanto $D$ é denso em $X$.

Seja $z \in D$. Então existe um $k \in \omega$ tal que $z \in Z_{k}$. Agora, para qualquer $n \in \omega \mathrm{e}$ $x \in Z_{n}$ temos $B_{\varepsilon_{n}(x)}(x) \cap D \subset V_{n}(x)$; e portanto $f\left[B_{\varepsilon_{n}(x)}(x) \cap D\right] \subset f\left[V_{n}(x)\right] \subset G_{n}(f(x))$. Portanto $f \mid D$ é contínua em $D=\bigcup_{n \in \omega} Z_{n}$.

Corolário 9.11. Seja $X$ é um espaço métrico. Então $X$ é de Baire se, e somente, se $X$ é Blumberg.

Agora, daremos um exemplo de um espaço que não é métrico, mas é Blumberg.

Proposição 9.12. $\mathbb{R}_{s}$ não é metrizável. Porém é de Blumberg.

Demonstração. Suponha que seja metrizável. Como a reta de Songefrey é separável, isso implicaria que ela possui base enumerável. Mas isso não é verdade.

Seja $f: \mathbb{R} \rightarrow \mathbb{R}$. Usando o fato de $\mathbb{R}$ na topologia usual ser Blumberg existe um subconjunto denso $D \subset \mathbb{R}$ tal que $f \mid D$ é contínua em $D$. Como os abertos da topologia de $\mathbb{R}_{s}$ contém abertos da topologia usual segue que $D$ é denso em $\mathbb{R}$ e $f \mid D$ é contínua em D. Portanto $\mathbb{R}_{s}$ é de Blumberg. 


\subsection{Um Exemplo de um Espaço Compacto Hausdorff e não Blumberg}

O quão longe Baire está de Blumberg? Sabemos que todo espaço localmente compacto Hausdorff não vazio é uma espaço de Baire. Com a construção desse exemplo obteremos um espaço de Baire que não é de Blumberg. Tentaremos uma caracterização dos espaços de Blumberg via Jogo de Choquet e esse resultado será central para mostrar que em geral não é verdade que Choquet implica Blumberg.

Para a construção desse espaço enunciaremos alguns resultados que não desmostraremos. Para conferir as demostrações omitidas veja [15].

Esse espaço será obtido em duas partes. A primeira assumindo $C H$ e a segunda parte negando $\mathrm{CH}$.

Definição 9.13. Uma função $f$ de um espaço topológico $X$ em um conjunto $Y$ é chamada de uma função $\delta$-fine se, e somente se, para cada $y \in Y, f^{-1}(y)$ é nunca denso em $X$.

Lema 9.14 $(C H)$. Suponha que $X$ é um espaço topológico onde os subconjuntos de $1^{a}$ categoria são nunca densos. Seja $\left\{f_{\alpha}: X \rightarrow[0,1]: \alpha<\omega_{1}\right\}$ uma família de $\omega_{1}$ funções $\delta$-fine em $X$. Então existe uma função $\delta$-fine $g: X \rightarrow[0,1]$ tal que para cada $\alpha<\omega_{1}$, $\left\{x: f_{\alpha}(x)=g(x)\right\}$ é nunca denso em $X$.

Definição 9.15. Um espaço topológico $X$ é extremamente desconexo se o fecho de todo aberto de $X$ é aberto.

Lema 9.16. Suponha que X seja um espaço topológico extremamente desconexo, completamente regular ${ }^{3}$ e Blumberg. Suponha que $g: X \rightarrow[0,1]$ é uma função $\delta$-fine. Então existe uma função $\delta$-fine continua $f: X \rightarrow[0,1]$ e um conjunto denso $D$ em $X$ tal que $f|D=g| D$.

Definição 9.17. Seja $X$ um espaço topológico. O peso é dado por

\footnotetext{
${ }^{3} \mathrm{O}$ espaço $X$ é completamente regular ou $T_{3 \frac{1}{2}}$ se para todo fechado $F \subset X$ e para todo $p \notin F$ existir uma função contínua $f: X \rightarrow[0,1]$ tal que $f(x)=1$ para todo $x \in F$ e $f(p)=0$.
} 


$$
w(X)=\omega \cdot \min \{\kappa: \mathcal{B} \text { é base para a topologia de } X \text { e }|\mathcal{B}|=\kappa\}
$$

Uma $\pi$-base $\mathcal{U}$ para um espaço $X$ é uma coleção de abertos não vazios de $X$ tal que se $V$ é um aberto não vazio arbitrário de $X$ então existe $U \in \mathcal{U}$ tal que $U \subset V$. O $\pi$-peso de um espaço $X$ é definido como:

$$
\pi \omega(X)=\omega \cdot \min \{\kappa: \text { existe uma } \pi \text {-base } \mathcal{U} \text { para } X \text { e }|\mathcal{U}|=\kappa\}
$$

A celularidade de um espaço $X$ é definido como:

$c(X)=\omega \cdot \sup \{\kappa: \mathcal{U}$ é uma coleção de abertos dois a dois disjuntos de $X$ e $|\mathcal{U}|=\kappa\}$.

Seja $C(X, Y)$ o conjunto de todas as funções continuas de $X$ em $Y . C(X)$ representa o conjunto das funções contínuas de $X$ a valores reais.

Lema 9.18. Seja $X$ um espaço topológico qualquer e seja $Y$ um espaço Hausdorff. Então $|C(X, Y)| \leq \pi(X)^{c(X) \cdot w(Y)}$.

Corolário $9.19(C H)$. Se $X$ é um espaço regular, então $C(X) \leq \pi(X)^{c(X)}$.

Teorema $9.20(\mathrm{CH})$. Seja $X$ um espaço topológico tal que:

(i) X é completamente regular e extremamente desconexo,

(ii) $\pi \omega(X) \leq \omega_{1}$ e c $c(X)=\omega$,

(iii) os subconjuntos de $1^{a}$ categoria de $X$ são nunca densos,

(iv) existe uma função $\delta$-fine definida em $X$ a valores reais.

Então X não é Blumberg.

Demonstração. Pelo Corolário 9.19Um Exemplo de um Espaço Compacto Hausdorff e não Blumbergteorema.9.19 existem no máximo $\left(2^{\omega}\right)^{\omega}=\omega_{1}$ funções contínuas $\delta$-fine. Pelo Lema 9.14Um Exemplo de um Espaço Compacto Hausdorff e não Blumbergteorema.9.14 existe uma função $\delta$-fine $g: X \rightarrow[0,1]$ tal que para qualquer função contínua $\delta$-fine $f$ de $X$ em $[0,1]$ temos que o conjunto $\{x \in X: f(x)=g(x)\}$ é nunca denso em $X$.

Agora, suponha que $X$ é Blumberg, por $(i)$, (iv) e o Lema 9.16Um Exemplo de um Espaço Compacto Hausdorff e não Blumbergteorema.9.16 existe uma função $h: X \rightarrow[0,1]$ 
contínua $\delta$-fine e um conjunto denso $D \subset X$ tal que $g|D=h| D$ e portanto $\{x \in X: h(x)=$ $g(x)\}$ é denso em $X$. Porém $h$ está inclusa nas $\omega_{1}$ funções contínuas $\delta$-fine, o que é um absurdo.

Agora, vamos construir um espaço que satisfaça todos os ítens do Teorema anterior.

Seja $\mathcal{B}$ a álgebra de Boole dos subconjuntos Lebesgue mensuráveis de [0,1], e $\mathcal{I}$ o ideal dos conjuntos de medida nula de $\mathcal{B}$. Vamos chamar de álgebra de medida enfraquecida a álgebra booleana completa ${ }^{4} \mathcal{B} / \mathcal{I}$. Seja $s(\mathcal{B} / \mathcal{I})$ o espaço de Stone da álgebra de medida enfraquecida. Para cada $B \in \mathscr{B}$, seja $[B]$ a classe de equivalência de $B \bmod \mathcal{I}$. Para cada $[B] \in \mathcal{B} / \mathcal{I}$, seja $[B]^{*}$ o aberto básico de $s(\mathcal{B} / \mathcal{I})$ associado a $[B]$. Seja $m$ a medida de Lebesgue.

(i) Por $s(\mathcal{B} / \mathcal{I})$ ser compacto e Hausdorff implica que $s(\mathcal{B} / \mathcal{I})$ é completamente regular.

Em [14] temos o seguinte resultado:

Proposição 9.21. Uma álgebra Booleana de subconjuntos abertos-fechados de um espaço zero-dimensional é completa se, e somente se, o espaço é extremamente desconexo.

Como $\mathcal{B} / \mathcal{I}$ é completa segue que o subcojunto de abertos-fechados de $s(\mathcal{B} / \mathcal{I})$ também o é.

Portanto $s(\mathcal{B} / \mathcal{I})$ é extremamente desconexo.

(ii) Para mostrar que $\pi \omega(s(\mathcal{B} / \mathcal{I})) \leq 2^{\omega}$ enunciaremos um Lema auxiliar e em seguida construiremos um conjunto denso com o tamanho desejado.

Definição 9.22. Seja A uma álgebra de Boole. Chamamos de densidade de A

$$
d(A)=\min \{\kappa: D \subset A \text { denso, }|D|=\kappa\}
$$

Lema 9.23. Seja A uma álgebra de Boole. Então $\pi \omega(s(A))=d(A)$.

\footnotetext{
${ }^{4}$ Seja $A$ uma álgebra booleana. Dizemos que $A$ é completa se para todo $M \subset A$ existir supM $\left(\sup M=\sum\{a: a \in M\}\right)$. Para ver que $\mathcal{B} / \mathcal{I}$ é completa veja o Corolário 1 da página 295 de [5].
} 
Demonstração. Seja $D \subset A$ denso tal que $|D|=d(A)$. Seja $U$ um aberto não vazio de $s(A)$. Então existe $a^{*} \subset U$ (por $\left\{a^{*}: a \in A\right\}$ ser base). Por $D$ ser denso em $A$ existe $d \in D$ tal que $d \leq a$. Assim $d^{*} \subset a^{* 5}$. Concluímos que $\left\{d^{*}: d \in D\right\}$ é uma $\pi$-base e portanto $\pi \omega(s(A)) \leq d(A)$.

Seja $\mathscr{B}$ uma $\pi$-base de $s(A)$ tal que $|\mathscr{B}|=\pi \omega(s(A))$. Seja $a \in A$. Logo existe $B \in \mathscr{B}$ tal que $B \subset a^{*}$. Como $B$ é aberto existe $b^{*} \subset B \subset a^{*}$ e assim $b \leq a$. Com isso $\left\{b: b^{*} \subset B\right.$ com $\left.B \in \mathscr{B}\right\}$ é um subconjunto denso em $A$ e portanto $\pi \omega(s(A)) \geq d(A)$.

Seja $\mathcal{F}=\left\{F \subset[0,1]: F\right.$ é $F_{\sigma}$ e $\left.m(F)>0\right\}$ e seja $\mathcal{F}^{\prime}=\{[F]: F \in \mathcal{F}\}$. O Teorema 1.19 de [4] diz que se $A$ é Lebesgue mensurável tal que $m(A)>0$ podemos escrever $A=H \cup N$ onde $H$ é $F_{\sigma}$ e $m(N)=0$. Logo $m(H)>0$. Portanto $\mathcal{F}^{\prime}$ é denso em $\mathcal{B} / \mathcal{I}$.

Afirmação 9.24. $|\mathcal{F}| \leq 2^{\omega}$.

Demonstração. Em primeiro lugar mostremos que a quantidade de abertos em $[0,1]$ é $2^{\omega}$. Seja $\left\{B_{n}\right\}_{n<\omega}$ uma base enumerável para $[0,1]$. Para cada $f \in \omega^{\omega}$ defina $V_{f}=\bigcup_{n<\omega} B_{f(n)}$. Com isso temos todas as formas possíveis de expressar abertos de $[0,1]$ em relação à base enumerável. Como para cada $f$ temos um aberto segue que a quantidade máxima de abertos é $2^{\omega}{ }^{6}$

Agora podemos definir os fechados como $F_{f}=[0,1] \backslash V_{f}$ e assim temos que a quantidade de fechados é no máximo $2^{\omega}$. Como cada elemento de $\mathcal{F}$ é a união enumerável de fechados conclui-se $|\mathcal{F}| \leq 2^{\omega}$.

Com isso mostramos que $\mathcal{F}^{\prime}$ é denso em $\mathcal{B} / \mathcal{I}^{7}$ e $\left|\mathcal{F}^{\prime}\right| \leq 2^{\omega}$ então $\pi \omega(s(\mathcal{B} / \mathcal{I})) \leq 2^{\omega}$.

Para mostrar que $c(s(\mathcal{B} / \mathcal{I}))=\omega$ é equivalente provar que $s(\mathcal{B} / \mathcal{I})$ satisfaz a c.c.c..

\footnotetext{
${ }^{5} u \in d^{*} \Rightarrow d \in u \Rightarrow a \in u \Rightarrow u \in a^{*}$.

${ }^{6}$ Pois $\left|\omega^{\omega}\right|=2^{\omega}$. Para ver isso, note que $2^{\omega} \leq \omega^{\omega}$ e $\omega^{\omega} \leq\left(2^{\omega}\right)^{\omega}=2^{\omega \cdot \omega}=2^{\omega}$.

${ }^{7} \mathrm{Na}$ verdade $\mathcal{B} / \mathcal{I}=\mathcal{F}^{\prime}$. Para perceber isso, veja a argumentação feita em (iii).
} 
Seja $m$ a medida de Lebesgue. Note que os elementos de $\mathcal{B} / \mathcal{I}$ possuem medida de Lebesgue positiva.

Seja $\mathscr{A}$ uma anticadeia em $s(\mathcal{B} / \mathcal{I})$ e suponha que não seja enumerável. Dentro de cada aberto de $\mathscr{A}$ existe um aberto básico contido no mesmo. Logo podemos considerar os elementos de $\mathscr{A}$ abertos básicos dois a dois disjuntos. Sejam $[B]^{*},[C]^{*} \in \mathscr{A}$, então $[B]^{*} \cap[C]^{*}=\emptyset$. Isso implica que $m(B \cap C)=0$, pois se $m(B \cap C)>0$ tomemos o aberto básico $[B \cap C]^{*}=\{u \in U l t(\mathcal{B} / \mathcal{I}): B \cap C \in u\}$, então $[B],[C] \in[B \cap C]^{*}$ então $[B \cap C] \subset[B]^{*} \cap[C]^{*} \operatorname{logo}[B]^{*} \cap[C]^{*} \neq \emptyset$.

Assim, temos uma família não enumerável $\left\{\left[B_{\alpha}\right]\right\}_{\alpha<\omega_{1}} \operatorname{com} m\left(B_{\alpha}\right)>0$ para todo $\alpha<\omega_{1}$ e $m\left(B_{\alpha} \cap B_{\beta}\right)=0$ se $\alpha \neq \beta$.

Segue da Proposição 0.20 de [4] que $m\left(\bigcup_{\alpha<\omega_{1}} B_{\alpha}\right)=\sum_{\alpha<\omega_{1}} m\left(B_{\alpha}\right)=\infty$. Porém, $m\left(\bigcup_{\alpha<\omega_{1}} B_{\alpha}\right) \leq m([0,1])=1$ o que é um absurdo.

(iii) Os subconjunto de $s(\mathcal{B} / \mathcal{I})$ de $1^{\circ}$ categoria são nunca densos.

Como foi dito anteriormente se $A$ é Lebesgue mensurável tal que $m(A)>0$ podemos escrever $A=H \cup N$ onde $H$ é $F_{\sigma}$ e $m(N)=0$. Como conjuntos $F_{\sigma}$ 's são borelianos, dado $[A]$ podemos tomar $B \in[A]$ boreliano tal que $m(A)=m(B)$. Com isso concluimos que para qualquer subconjunto de $\mathcal{B} / \mathcal{I}$ podemos tomar os representantes das classes como sendo borelianos.

Em [5] temos o seguinte resultado para a álgebra de Boole de subconjuntos boréis mensuráveis:

Teorema 9.25. Todo conjunto boreliano de $1^{\circ}$ categoria é nunca denso.

Portanto, pelas considerações feitas, temos o resultado desejado. 
(iv) Existe uma função $\delta$-fine a valores reais. Sejam

$$
\begin{aligned}
\mathscr{B}_{0} & =\left\{\left[B_{0}^{0}\right]:=\left[\left[0, \frac{1}{2}\right]\right],\left[B_{1}^{0}\right]:=\left[\left[\frac{1}{2}, 1\right]\right],\left[B_{2}^{0}\right]:=\emptyset,\left[B_{3}^{0}\right]:=\emptyset, \ldots\right\} \\
\mathscr{B}_{1} & =\left\{\left[B_{0}^{1}\right]:=\left[\left[0, \frac{1}{4}\right]\right], \ldots,\left[B_{3}^{1}\right]:=\left[\left[\frac{3}{4}, 1\right]\right],\left[B_{4}^{1}\right]:=\emptyset,\left[B_{5}^{1}\right]:=\emptyset, \ldots\right\} \\
\mathscr{B}_{2} & =\left\{\left[B_{0}^{2}\right]:=\left[\left[0, \frac{1}{2^{3}}\right]\right],\left[B_{1}^{2}\right]:=\left[\left[\frac{1}{2^{3}}, \frac{2}{2^{3}}\right]\right], \ldots,\left[B_{7}^{2}\right]:=\left[\left[\frac{7}{2^{3}}, 1\right]\right],\left[B_{8}^{2}\right]:=\emptyset,\left[B_{9}^{2}\right]:=\emptyset, \ldots\right\} \\
\vdots & \mathscr{B}_{n}=\left\{\left[B_{0}^{n}\right]:=\left[\left[0, \frac{1}{2^{n+1}}\right]\right], \ldots,\left[B_{2^{n+1}-1}^{n}\right]:=\left[\left[\frac{2^{n+1}-1}{2^{n+1}}, 1\right]\right],\left[B_{2^{n+1}}^{n}\right]:=\emptyset,\left[B_{2^{n+1}+1}^{n}\right]:=\emptyset, \ldots\right\}
\end{aligned}
$$

Observe que os representantes das classes de $\mathscr{B}_{0}$ tem medida $1 / 2$ os de $\mathscr{B}_{1}$ tem medida $1 / 4$ e os representantes das classes de $\mathscr{B}_{n}$ tem medida $1 / 2^{n+1}$.

Para cada $f \in \omega^{\omega}$, seja $N(f)=\bigcap\left\{\left[B_{f(n)}^{n}\right]^{*}: n<\omega\right.$ e $\left.\left[B_{f(n)}^{n}\right] \in \mathscr{B}_{n}\right\}$. Note que para cada $f$ que tomamos é o mesmo que pegar um elemento de cada $\mathscr{B}_{n}$ e depois interceptar todos eles. Note que pela construção dos $\mathscr{B}_{n}$ 's o número de funções que escolhem esses elementos é $2^{\omega}$.

Seja $\mathscr{F}=\left\{N(f): f \in \omega^{\omega}\right\}$. Podemos ver que $|\mathscr{F}| \leq 2^{\omega}$.

Afirmação 9.26. $\bigcup \mathscr{F}=s(\mathcal{B} / \mathcal{I})$

Demonstração. Basta mostrar que $s(\mathcal{B} / \mathcal{I}) \subset \bigcup \mathscr{F}$. Seja $u \in s(\mathcal{B} / \mathcal{I})$.

Mostremos o seguinte resultado auxiliar: Para cada $\mathscr{B}_{n}$ existe $[A] \in \mathscr{B}_{n}$ tal que $A \in u$. Suponha que exista $k<\omega$ tal que para todo $B \in \mathscr{B}_{k}$ temos $B \notin u$. Por $u$ ser ultrafiltro temos que $B^{c} \in u$. Como $\mathscr{B}_{k}$ é finito temos que $\emptyset=B_{0}^{c} \cap B_{1}^{c} \cap \cdots \cap B_{2^{k+1}}^{c} \in$ $u$. O que é um absurdo.

Assim, existe $f \in \omega^{\omega}$ tal que $B_{f(n)}^{n} \in u$ então $u \in\left[B_{f(n)}^{n}\right]^{*}$ para todo $n<\omega$ e portanto $u \in \bigcap_{n<\omega}\left[B_{f(n)}^{n}\right]^{*}$. Logo, $u \in N(f)$ para algum $f \in \omega^{\omega}$. 
Seja $\left\{F_{\alpha}: \alpha<2^{\omega}\right\}$ uma enumeração de $\mathscr{F}$. Para cada $\alpha<2^{\omega}$, seja $F_{\alpha}^{\prime}=F_{\alpha} \backslash$ $\bigcup\left\{F_{\beta}: \beta<\alpha\right\}$. Se $\left\{y_{\alpha}: \alpha<2^{\omega}\right\}$ for uma enumeração de $[0,1]$, então defina $g: s(\mathscr{B} / \mathscr{I}) \rightarrow[0,1]$ como $g(u)=y_{\alpha}$ onde $\alpha$ é o índice do único $F_{\alpha}^{\prime}$ onde $u \in F_{\alpha}^{\prime}$. Com isso $g^{-1}\left(\left\{y_{\alpha}\right\}\right) \in N(f)$ para algum $f \in \omega^{\omega}$.

Note que $\operatorname{int}(\overline{N(f)})=\emptyset$. Pois, para todo $[a]^{*}$ existe um aberto $[b]^{*} \subset[a]^{*}$ tal que $[b]^{*} \cap\left(\bigcap_{n<\omega}\left[B_{f(n)}^{n}\right]^{*}\right)^{8}$. Com isso $\overline{N(f)}=\emptyset$ e então $\operatorname{int}(\overline{N(f)})=\emptyset$.

Portanto $g$ é $\delta$-fine.

Corolário $9.27(\mathrm{CH}) . s(\mathcal{B} / \mathcal{I})$ é compacto, Hausdorff e não é Blumberg.

Vamos agora considerar a parte com $\neg C H$.

Definição 9.28. Um espaço no qual a sua topologia é dada pela topologia induzida por uma ordem total é chamado de LOTS (Linearly Ordered Topological Space).

Definição 9.29. Uma coleção $\mathscr{C}$ de abertos de um espaço topológico $X$ é chamada de óbvia se existe um subconjunto aberto $V \subset X$ tal que para todo $x \in V$ e para todo $\mathscr{C}^{\prime} \subset \mathscr{C}$ se $x \in \bigcap \mathscr{C}^{\prime}$ implica que existe um aberto não vazio $W \subset V$ tal que $W \subset \bigcap \mathscr{C}^{\prime}$.

Teorema 9.30. Se X é Baire e LOTS, então X não é Blumberg se, e somente se, existe um aberto $U \subset X$ tal que

(i) U é a união de no máximo $2^{\omega}$ conjuntos nunca densos, e

(ii) toda coleção enumerável de subconjuntos abertos de $U$ é óbvia.

Teorema 9.31. Existe um compacto LOTS com as seguintes propriedades:

(a) é a união de $\omega_{2}$ subconjuntos nunca densos,

(b) toda coleção enumerável de subconjuntos abertos é óbvia.

Corolário 9.32. Assumindo $2^{\omega} \geq \omega_{2}$. O espaço em questão do Teorema 9.31Um Exemplo de um Espaço Compacto Hausdorff e não Blumbergteorema.9.31 é um espaço compacto LOTS que não é Blumberg.

\footnotetext{
${ }^{8}$ Basta tomar $a \in \mathscr{B}_{m}$ para $m<\omega$ suficientemente grande de modo que $a \cap B_{f(m)}^{m}=\emptyset$.
} 
Demonstração. Segue do Teorema 9.30Um Exemplo de um Espaço Compacto Hausdorff e não Blumbergteorema.9.30.

Teorema 9.33. Existe um espaço compacto Hausdorff que não é de Blumberg.

Demonstração. Seja $\tilde{L}$ o espaço o espaço obtido mediante ao Teorema 9.31Um Exemplo de um Espaço Compacto Hausdorff e não Blumbergteorema.9.31. Seja $X$ a união disjunta dos elementos de $s(\mathcal{B} / \mathcal{I})$ e $\tilde{L}^{9}$. Se $X$ é Blumberg então $s(\mathcal{B} / \mathcal{I})$ e $\tilde{L}$ também seriam Blumberg. Mas pelos Corolários 9.27Um Exemplo de um Espaço Compacto Hausdorff e não Blumbergteorema.9.27 e 9.32Um Exemplo de um Espaço Compacto Hausdorff e não Blumbergteorema.9.32, independente do valor de $2^{\omega}, s(\mathcal{B} / \mathcal{I})$ e $\tilde{L}$ não podem ser ambos Blumberg.

\subsection{Caracterização por Jogos}

No capítulo 6 vimos alguns tipos de Jogos Topológicos e o ganho na linguagem de jogos é deixar algo "complicado" com uma formulação mais simples. Para tal, tentamos caracterizar os espaços de Blumberg via o Jogo de Choquet. O curioso nisso é que tínhamos somente uma resposta para um lado das implicações (Blumberg não implica Choquet). Ao apresentar isso na Segunda Semana de Topologia Geral e Teoria de Conjuntos (2nd Set Theory and General Topology Week) em Salvador BA o Professor Frank Tall ${ }^{10}$ nos informou do artigo [15] onde é construído um espaço compacto Hausdorff não Blumberg. Esse espaço é um exemplo fundamental para mostrar que Choquet não implica Blumberg. Mas a busca por uma caracterização continua...

Até agora temos:

- Blumberg $\Rightarrow$ Baire,

- Baire $\nRightarrow$ Blumberg ${ }^{11}$,

- Métrico Blumberg $\Leftrightarrow$ Métrico de Baire,

\footnotetext{
${ }^{9} X=(\{0\} \times s(\mathcal{B} / \mathcal{I})) \cup(\{1\} \times \tilde{L})$

${ }^{10}$ Professor da University of Toronto - St. George Campus.

${ }^{11} \mathrm{O}$ espaço $X$ construido na seção anterior é um exemplo de um espaço que é de Baire, mas não é de Blumberg.
} 
- Choquet $\Rightarrow$ Baire.

Agora podemos nos perguntar se :

- Blumberg $\Rightarrow$ Choquet ou,

- Choquet $\Rightarrow$ Blumberg.

Infelizente o Jogo de Choquet não caracteriza os Espaços de Blumberg. Vejamos o porque:

Suponha que Blumberg implica Choquet. Por [3] e [10] existe um exemplo de um espaço métrico de Baire no qual o quadrado não é de Baire. Chame esse espaço de $M$. Como $M$ é métrico de Baire, é também Blumberg. Assim, $M$ é Choquet. Logo $M^{2}$ é Choquet. Portanto, $M^{2}$ é de Baire. Temos assim um absurdo.

Agora, para mostrar que a outra implicação não é verdadeira, na seção anterior construímos um espaço $X$ compacto Hausdorff que não é Blumberg. Pela Proposição 6.6Jogo de Choquet (Banach Mazur)teorema.6.6 temos que $X$ é Choquet, porém não é Blumberg. 


\section{Considerações finais}

Espero que esse trabalho seja útil para se conhecer o Axioma de Martin, o Princípio Diamante e a Hipótese de Suslin e algumas aplicações em topologia geral. Parte dessa dissertação é uma espécie de preparação para a compreensão da aplicação da técnica de forcing, citada no capítulo 3. Porém, vimos que isso não depende exclusivamente de tal técnica, pois podemos gerar axiomas a partir de outros como foi visto no capítulo 5 . 


\section{Referências Bibliográficas}

[1] Babinkostova, L. Set Theory and Its Applications: Annual Boise Extravaganza in Set Theory, 1995-2010, Boise, Idaho. Contemporary Mathematics. American Mathematical Society, 2011.

[2] Blumberg, H. New properties of all real functions. Trans. Amer. Math. Soc. 24, 2 (1922), 113-128.

[3] Cohen, P. E. Products of Baire spaces. Proc. Amer. Math. Soc. 55, 1 (1976), $119-124$.

[4] Folland, G. Real analysis: modern techniques and their applications. Pure and applied mathematics. Wiley, 1999.

[5] Givant, S., And Halmos, P. Introduction to Boolean algebras. Undergraduate texts in mathematics. Springer, 2009.

[6] Goffman, C., Nishiura, T., And Waterman, D. Homeomorphisms in analysis. Mathematical surveys and monographs. American Mathematical Society, 1997.

[7] Haworth, R. C., And MCCoy, R. A. Baire spaces. Dissertationes Math. (Rozprawy Mat.) 141 (1977), 73.

[8] JECH, T. Set theory. Springer monographs in mathematics. Springer, 2003.

[9] Kechris, A. Classical descriptive set theory. Graduate texts in mathematics. Springer-Verlag, 1995. 
[10] Krom, M. R. Cartesian products of metric Baire spaces. Proc. Amer. Math. Soc. $42(1974), 588-594$.

[11] Kunen, K. Set theory: an introduction to independence proofs. Studies in logic and the foundations of mathematics. North-Holland Pub. Co., 1980.

[12] Moore, J. T. Some of the combinatorics related to Michael's problem. Proc. Amer. Math. Soc. 127, 8 (1999), 2459-2467.

[13] Telgársky, R. Topological games: on the 50th anniversary of the Banach-Mazur game. Rocky Mountain J. Math. 17, 2 (1987), 227-276.

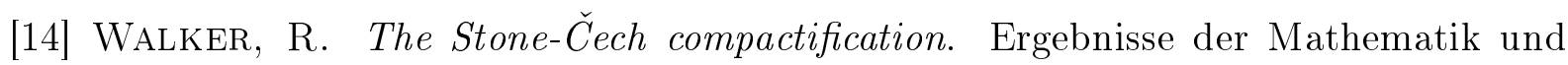
ihrer Grenzgebiete. Springer-Verlag, 1974.

[15] Weiss, W. A. R. The Blumberg problem. Trans. Amer. Math. Soc. 230 (1977), $71-85$. 


\section{Índice Remissivo}

$\Delta$ - sistema, 92

$\pi$-base, 118

$\pi$-peso, 118

álgebra de Boole, 21

densidade de uma, 119

árvore, 13

árvore bem podada, 20

árvore de

Aronszajn, 17

Suslin, 21

árvore que sempre ramifica, 72

ínfimo, 10

ínfimo de um conjunto de ordinais, 11

$1^{\mathrm{a}}$ categoria

subconjunto de, 110

$2^{\mathrm{a}}$ categoria

subconjunto de, 110

anticadeia, 10

no sentido de árvore, 15

Baire

espaço de, 33

teorema de, 33 base para uma topologia, 27

bem ordenado

$$
\text { conjunto, } 9
$$

Blumberg

espaço de, 109

boa ordem, 9

cadeia, 10

cardinal

número, 12

regular, 13

sucessor, 12

celularidade, 118

Choquet

espaço de, 80

jogo de, 78

club, 39

$\operatorname{Club}(\mu), 40$

cobertura, 30

cobertura aberta, 30

coleção de abertos óbvia, 123

conjunto aberto, 27

conjunto estacionário, 41 
conjunto fechado, 30

conjunto fechado sob uma função, 42

conjunto ordenado, 8

conjunto parcialmente ordenado, 8

conjunto pré-ordenado, 7

conjunto totalmente ordenado, 8

convergência de ultrafiltro, 102

denso

conjunto, 10

elemento máximo, 9

elemento mínimo, 9

elemento maximal, 9

elemento minimal, 9

espaço $\sigma$-compacto, 89

espaço 0-dimensional, 101

espaço compacto, 30

espaço conexo, 28

espaço de Lindelöf, 31

espaço extremamente desconexo, 117

espaço localmente compacto, 30

espaço regular, 32

espaço topológico, 27

espaço topológico $T_{1}, 28$

família dominante, 50

família ilimitada, 50

fecho de um conjunto, 31

filtro, 23

completo, 40

função $\delta$-fine, 117 função finita, 42

função ilimitada, 12

função n-ária em $A, 42$

incompatíveis

elementos, 10

Lema de Zorn, 10

Lema do "Pressing Down", 44

limitante inferior, 9

limitante superior, 9

Linearly Ordered Topological Space (LOTS), 123

localmente finita

família, 111

Martin

axioma de, 36

Menger

espaço de, 86

jogo de, 86

propriedade de, 86

nunca denso

subconjunto, 110

ordem, 7

topologia da, 28

ordem $\leq^{*}, 49$

ordem estrita, 8

ordem reversa, 16

ordinal

cofinalidade de um, 12 
limite, 11

número, 11

sucessor, 11

Oxtoby

teorema de, 79

peso de um espaço topológico, 117

ponto aderente, 31

ponto aderente à um ultrafiltro, 102

ponto relativamente forte com um conjunto,

113

pré ordem, 7

Princípio $\diamond, 44$

propriedade da intersecção finita (p.i.f.), 24, supremo, 10

supremo de um conjunto de ordinais, 11

Suslin

árvore de, 60

reta de, 56

Teorema da indução transfinita, 11

topologia fraca, 29

topologia produto, 29

transitivo

conjunto, 10

Tychonoff

teorema de, 103

30

ultrafiltro, 25

raíz de uma árvore, 13

ramo de uma árvore, 13

Rothberger

espaço de, 83

jogo de, 82

propriedade de, 82

separável

espaço, 32

Sorgenfrey

reta de, 28

Stone

espaço de, 100

sub-árvore, 17

sub-árvore de $T$ abaixo de $\alpha, 70$

sucessor, 15 